Aus dem Institut für Ethik, Geschichte und Theorie der Medizin der Ludwig-Maximilians-Universität München Vorstand: Prof. Dr. Georg Marckmann, MPH

\title{
Beratung zur Patientenverfügung
}

Eine empirisch-ethische Untersuchung der Beratung durch ausgewählte Anbieter in der Region München

\author{
Dissertation \\ zum Erwerb des Doktorgrades der Humanbiologie \\ an der Medizinischen Fakultät der \\ Ludwig-Maximilians-Universität München
}

\author{
vorgelegt von \\ Sabine Petri \\ aus Lingen/Ems
}


Mit Genehmigung der Medizinischen Fakultät der Universität München

Berichterstatter: $\quad$ Prof. Dr. med. Georg Marckmann

Mitberichterstatter: $\quad$ Prof. Dr. med. Claudia Bausewein

Priv. Doz. Dr. med. Anneser

Dekan: $\quad$ Prof. Dr. med. Dr. h.c. M. Reiser, FACR, FRCR

Tag der mündlichen Prüfung: 23.07.2015 
Meinen Eltern 


\section{Zusammenfassung}

Aufgrund des medizinischen Fortschritts hat sich die Zahl der Behandlungsoptionen und damit der Therapieentscheidungen, insbesondere auch am Lebensende vervielfacht. Die Berücksichtigung des Willens des Patienten bei den häufig komplexen Entscheidungen ist ethisch wie rechtlich geboten und entspricht dem Wunsch der meisten Menschen nach Wahrung ihrer Selbstbestimmung. Vielfach ist eine aktive Beteiligung der Patienten im Zeitpunkt der Therapieentscheidung jedoch nicht möglich, da die dafür erforderliche Einwilligungsfähigkeit nicht mehr gegeben ist. Von der Möglichkeit durch eine Patientenverfügung Vorsorge für diese Situation zu treffen und so ihrem Selbstbestimmungsrecht Ausdruck zu verleihen, machen viele Menschen nicht oder nicht in tragfähiger Weise Gebrauch. Maßgeblichen Einfluss auf die Prävalenz und die Qualität der Vorsorge durch eine Patientenverfügung haben die zahlreichen Personen, Berufsgruppen oder Organisationen, die Beratungen zur Patientenverfügung durchführen. Über Struktur, Inhalt und Umfeld dieser Beratungen ist, ebenso wie über die Wünsche der Berater im Hinblick auf die Weiterentwicklung der Beratung wenig bekannt.

Durch die vorliegende explorative Fragebogenstudie wurde erstmals ein Einblick in die Beratungspraxis von 198 Beratern im Raum München gewonnen. Die Teilnehmer berieten in ihren Funktionen als Hausärzte, Notare, Überleitungsfachkräfte und Mitarbeiter von Hospizvereinen.

Die Unterschiedlichkeit der beratenden Berufsgruppen spiegelte sich auch in unterschiedlichen Beratungsstrukturen und -Inhalten wieder, die aber dennoch einige übergreifende Schwerpunkte erkennen ließen:

Die meistens unentgeltlichen Beratungen strukturierten sich überwiegend in ein bis zwei Gespräche, die bis zu 30 Minuten dauerten. Die Initiative zur Beratung ging primär von der beratenen Person selbst aus. Die größte Gruppe der Ratsuchenden bildeten 61 bis 80jährige Menschen. Ärzte und Notare berieten vornehmlich gesunde, Überleitungsfachkräfte und Mitarbeiter von Hospizvereinen überwiegend schwer kranke Menschen. Auf die Möglichkeit der Teilnahme des zukünftigen Vertreters des Ratsuchenden an der Beratung wiesen über zwei Drittel der Mitglieder aller nicht- 
ärztlichen Beratergruppen hin. Bei den Ärzten gab nur die Hälfte diesen Hinweis. Diese Personen nahmen nur bei weniger als einem Fünftel der ärztlichen Beratungen teil, aber bei über der Hälfte der nichtärztlichen Beratungen. Nur maximal die Hälfte der Beratungen durch die verschiedenen Gruppen endete mit einer fertigen Patientenverfügung. Besondere Bedeutung kam dem Formular des Bayerischen Staatsministeriums der Justiz als Vorlage für die Beratung zu.

Inhaltlich umfassten die Beratungen überwiegend die Themen Flüssigkeit und Ernährung, unheilbare tödlich verlaufende Krankheit, irreversible Bewusstlosigkeit und akuter medizinischer Notfall, sowie Hinweise zur Aufbewahrung der Patientenverfügung. Fragen zu psychiatrischen Erkrankungen und Organspende fanden weniger häufig Berücksichtigung. Die Themen Vorsorgevollmacht und Betreuungsverfügung wurden zwar überwiegend, aber nicht immer angesprochen. Hinweise auf die Möglichkeit eines Notfallplanes wurden von etwa der Hälfte der Mitglieder der beratenden Gruppen gegeben. In die Erstellung der Notfallpläne waren Hausärzte und Überleitungsfachkräfte deutlich weniger eingebunden als die Mitarbeiter der Hospizvereine und Notare nicht.

Die Wünsche der nichtärztlichen Berater richteten sich primär auf eine verbesserte Zusammenarbeit im Einzelfall mit dem Arzt, bei Notaren auf ein Feedback aus der Praxis zur Anwendung der Patientenverfügung. Daneben wurde der Wunsch nach verstärkter Information im rechtlichen, medizinischen und ethischen Bereich benannt. Die für die Beratung benötigten Kenntnisse erwarben die Berater, mit Ausnahme der Ärzte, die sich vornehmlich im Selbststudium weiterbildeten, überwiegend durch Schulungen.

Klare Beratungsprofile der einzelnen Beratergruppen ließen sich aufgrund der Heterogenität der Antworten der Berater innerhalb der Gruppen nicht erstellen.

Die beschriebene Situation der Beratung enthält bereits zahlreiche Elemente umfassender Advance Care Planning Programme (ACP). Die Heterogenität der Beratungsstrukturen und -inhalte innerhalb und zwischen den Gruppen lässt jedoch darauf schließen, dass die best practice derartiger Programme vielfach noch nicht erreicht ist. 
Aus diesem durch die Studie erhaltenen Einblick in die aktuelle Beratungssituation, den aus der Literatur gewonnenen Erkenntnissen und mit Blick auf etablierte ACP Programme konnten folgende Hinweise für die Weiterentwicklung der Beratung zur Patientenverfügung abgeleitet werden:

Die Beratung sollte, insbesondere durch (geschulte) Ärzte, vermehrt aktiv, gegebenenfalls sogar routinemäßig angeboten werden und nicht, wie es bisher überwiegend geschieht, der Initiative des zu Beratenden überlassen bleiben. Eine verstärkte und frühere Einbeziehung des zukünftigen Vertreters könnte dazu beitragen, dass dieser seiner Aufgabe der Ermittlung und Umsetzung des Patientenwillens besser nachkommen kann und zugleich seine damit verbundene psychische Belastung verringert wird. Regelmäßige Hinweise auf die Möglichkeit einer Notfallplanung, sowie die vermehrte Erstellung von derartigen Plänen würden die Umsetzung des Patientenwillens auch in Notfallsituationen unterstützen und ungewünschte Behandlungen reduzieren. Zur Förderung von Mindestqualität und Transparenz der Beratung wäre die Entwicklung oder Weiterführung von Standards oder Guidelines durch die Gruppen oder auch gruppenübergreifend anzuregen.

Um die genannten Perspektiven der Weiterentwicklung umsetzen zu können, ist vor allem der Bereitschaft der betroffenen Personen, Berufsgruppen und Institutionen hierzu, sowie die Klärung der jeweiligen Rollenverständnisse und Beraterprofile erforderlich. Daneben müssten die Fragen zur Finanzierung der Vorausplanung beantwortet werden. Unerlässlich erscheinen qualitativ hochwertige Schulungen, um Barrieren abzubauen und notwendiges Wissen zu vermitteln. Flankierend zu diesen Maßnahmen sollte durch Öffentlichkeitsarbeit und Forschung weiteres Wissen um die Grundlagen und Bedeutung der Vorausplanung in der Bevölkerung und in Fachkreisen generiert werden. 


\section{Inhaltsverzeichnis}

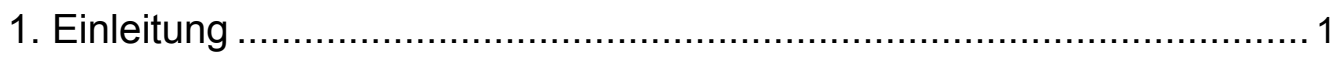

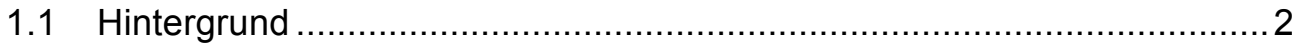

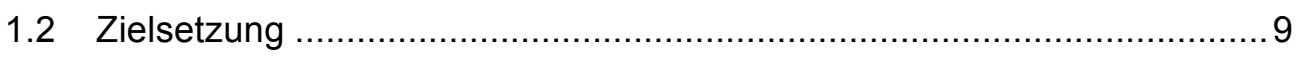

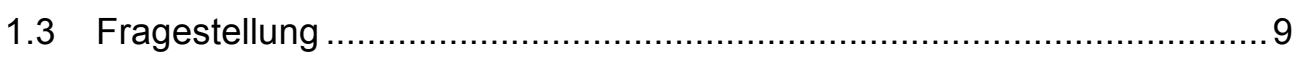

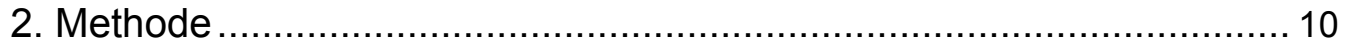

2.1 Planung und Vorbereitung der Datenerhebung .................................... 10

2.1.1 Entwicklung des Forschungsinstrumentes .................................. 10

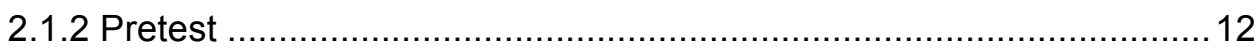

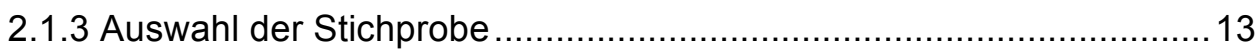

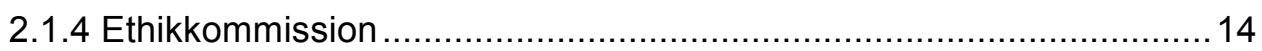

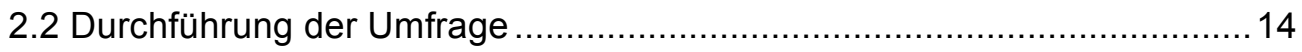

2.2.1 Rekrutierung der Teilnehmer ..................................................... 14

2.2.2 Studienverlauf....................................................................... 16

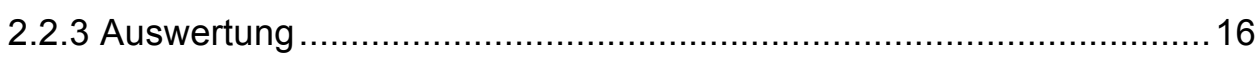

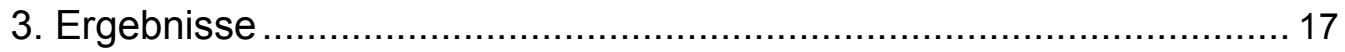

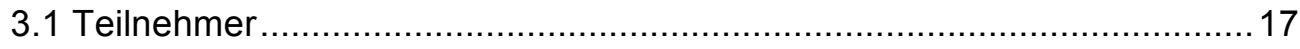

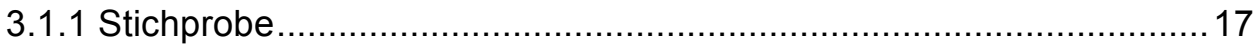

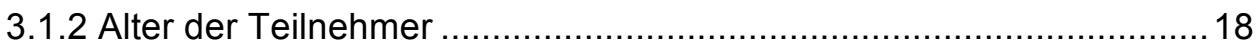

3.1.3 Verteilung der Geschlechter .................................................... 18

3.1.4 Jahre der ausgeübten Beratertätigkeit.............................................. 19

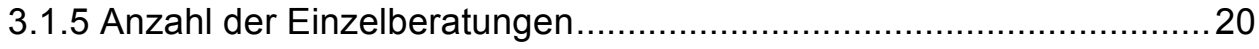

3.2 Inhalt und Struktur der Beratungstätigkeit ......................................... 20

3.2.1 Veranlasser der Beratung .........................................................20

3.2.2 Gesundheitszustand der Ratsuchenden........................................ 24

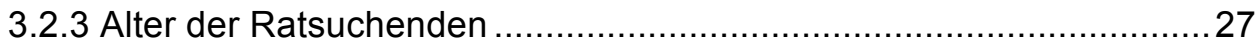

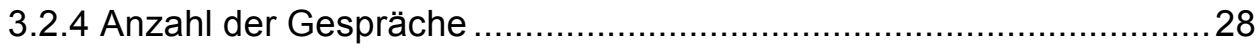

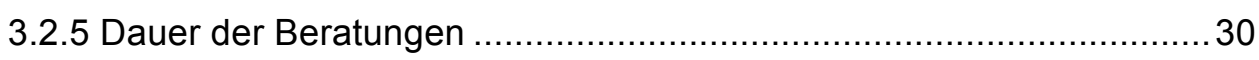

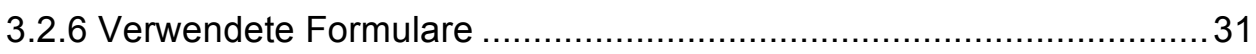

3.2.7 Verwendung von durch den Ratsuchenden mitgebrachten Formularen .34

3.2.8 Fertigstellung der Patientenverfügung ........................................ 36

3.2.9 Hinweise für die Aufbewahrung der Patientenverfügung .................. 37

3.2.10 Inhalt der Beratungsgespräche im Einzelnen .................................39

3.2.11 Information zu anderen Vorsorgedokumenten ............................. 42 
3.2.12 Hinweis auf Möglichkeit eines Notfallplanes und dessen tatsächliche

Erstellung .43

3.2.13 Einbeziehung des zukünftigen Vertreters ...................................45

3.2.14 Auswirkung der Teilnahme des Vertreters................................... 47

3.2.15 Hinweis auf die Möglichkeit einer Organspende .............................. 49

3.2.16 Themen im Hinblick auf eine Organspende.................................50

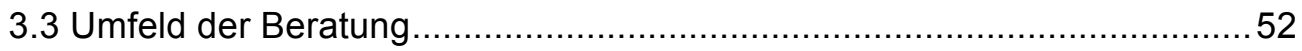

3.3.1 Einbeziehung der Berater in die Entscheidungsfindung bei

Einwilligungsunfähigkeit der Beratenen .52

3.3.2 Häufigkeit des Verweises an andere Berufsgruppen zur ergänzenden Beratung .53

3.3.3 Berufsgruppen, an die zur ergänzenden Beratung verwiesen wird ....54

3.3.4 Wunsch nach verstärkter Zusammenarbeit mit bestimmten

Berufsgruppen 56

3.3.5 Bereiche, in denen mehr Austausch/Information gewünscht wird ......57

3.3.6 Gewünschte Verbesserung des Austausches, der Zusammenarbeit, der Information. .59

3.3.7 Routinemäßiges Angebot einer Beratung zur Patientenverfügung ....61

3.3.8 Erwerb der Kenntnisse für die Beratung ........................................62

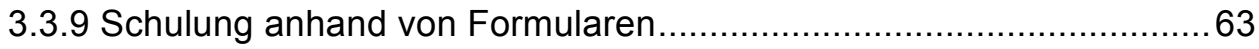

3.3.10 Benennung der Schulungsformulare .......................................... 64

3.3.11 Kostenpflicht der Beratung .................................................... 65

3.3.12 Vergütung für den Berater ......................................................66

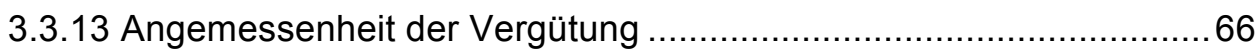

3.3.14 Angemessene Höhe der Vergütung ….......................................67

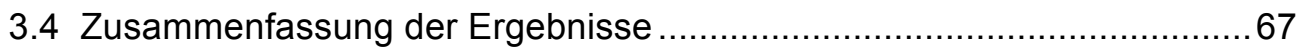

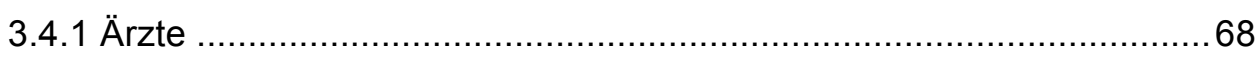

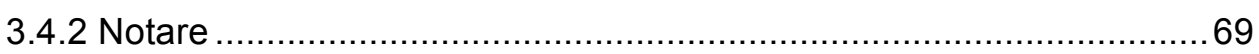

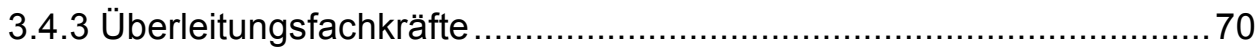

3.4.4 Mitarbeiter der Hospizvereine ......................................................... 71

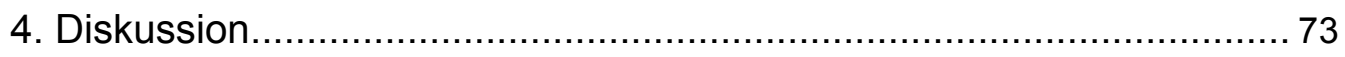

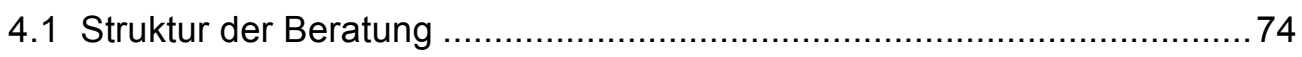

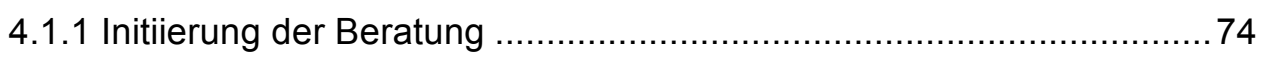

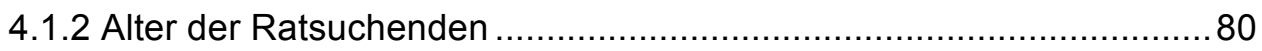

4.1.3 Gesundheitszustand der Ratsuchenden....................................... 81

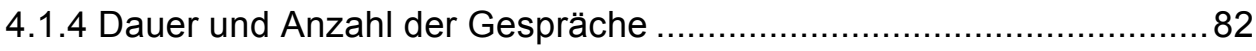

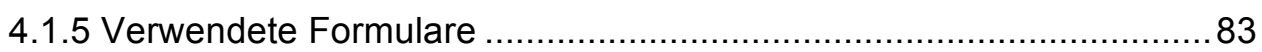


4.1.6 Vorsorgevollmacht und Betreuungsverfügung.... .84

4.1.7 Einbeziehung des Vertreters der Ratsuchenden .............................84

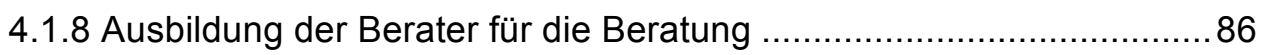

4.1.9 Fertig erstellte Patientenverfügungen ............................................ 86

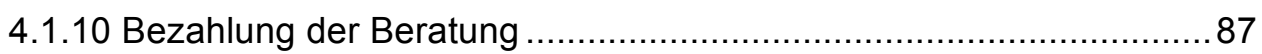

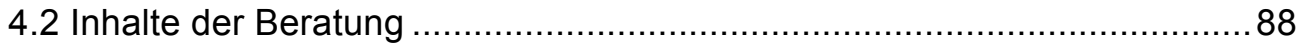

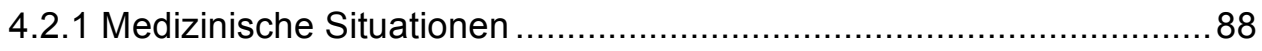

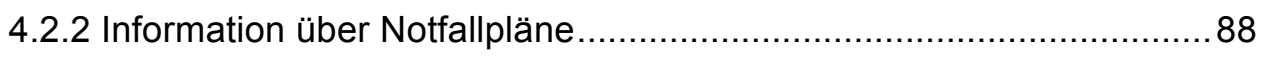

4.2.3 Erarbeitung von Notfallplänen ................................................... 89

4.2.4 Hinweis auf die Möglichkeit einer Organspende................................90

4.2.5 Hinweise zur Aufbewahrung der Patientenverfügung.......................90

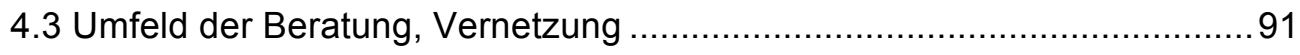

4.3.1 Verweis zur ergänzenden Beratung …........................................ 91

4.3.2 Einbeziehung der Berater bei medizinischen Entscheidungen...........94

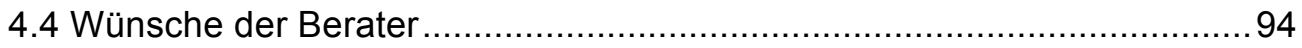

4.4.1 Wünsche an die Weiterentwicklung der Beratung ............................94

4.5 Erwerb der für die Beratung benötigten Kenntnisse ............................... 96

4.6 Zusammenfassung der Diskussion................................................. 97

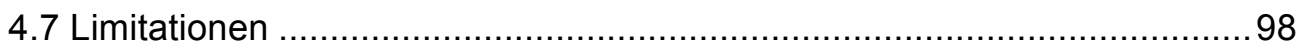

5. Ausblick: Perspektiven für die Weiterentwicklung der Beratung zur Patientenverfügung ................................................................. 100

5.1 Vermehrte Initiative der Berater, aktives Beratungsangebot ..................100

5.2 Verstärkte Einbeziehung zukünftiger Vertreter in die Beratung............... 101

5.3 Mindestanforderungen an Beratungsinhalt........................................ 102

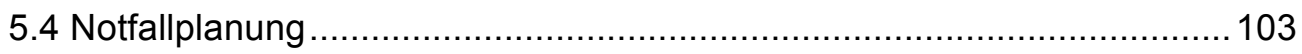

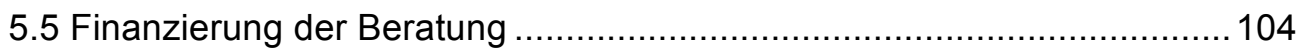

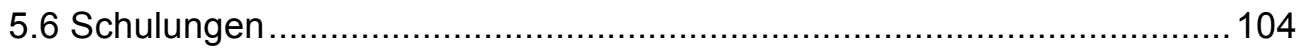

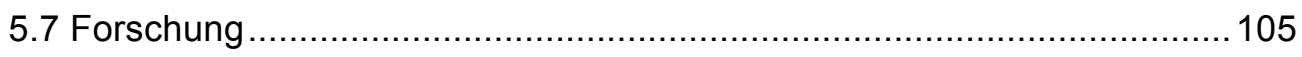

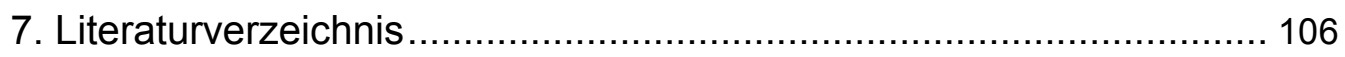

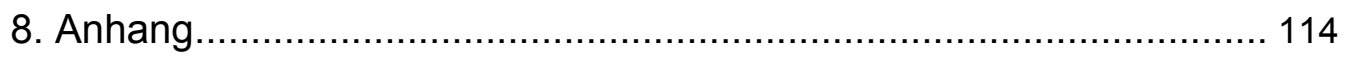

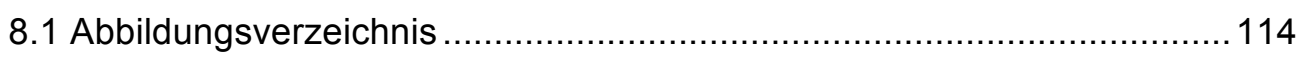

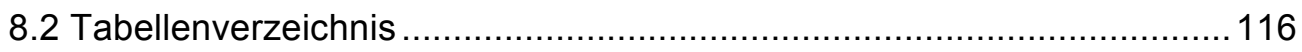

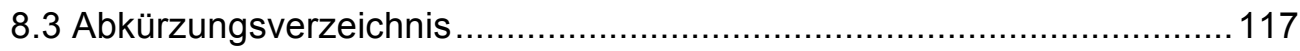

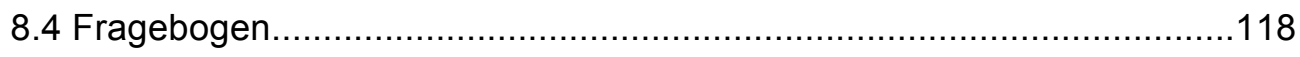

9. Danksagung 


\section{Einleitung}

Der objektive Bedarf und das individuelle Bedürfnis an ethisch wie rechtlich tragfähigen Entscheidungen zur Therapiewahl im Fall der Nichteinwilligungsfähigkeit der Patienten ${ }^{1}$ sind in den letzten Jahren enorm gestiegen.

Durch den medizinischen Fortschritt haben sich die Therapieoptionen zur Behandlung kranker Menschen vervielfacht. Wo früher die Akzeptanz eines unausweichlichen Todes gefordert war, besteht heute ein großer Bedarf an komplexen Entscheidungen über den Beginn oder die Fortsetzung lebensverlängernder Therapien (Schildmann et al. 2014; Silveira et al. 2010; Sprung et al. 2003; van der Heide et al. 2003).

Die Beteiligung der Patienten an diesen Entscheidungen ist nicht nur rechtlich gefordert und ethisch geboten, sondern entspricht auch dem Bedürfnis der meisten Menschen in unserer Gesellschaft nach Selbstbestimmung, Fürsorge und Wahrnehmung als einzigartiges Individuum (Dörner 2005).

Vielfach können die anstehenden Entscheidungen jedoch nicht unter aktiver Mitwirkung des Patienten getroffen werden, da dieser seine Einwilligungsfähigkeit zum Zeitpunkt der Entscheidung bereits verloren hat (Silveira et al. 2010; van der Heide et al. 2003).

Die gesetzlich geregelten Vorsorgeinstrumente Patientenverfügung, Vorsorgevollmacht und Betreuungsverfügung sollen dem Einzelnen ermöglichen, Vorsorge für den Fall des Verlustes der Einwilligungsfähigkeit im Krankheitsfall zu treffen und so sein Selbstbestimmungsrecht auszuüben. Sie können ein wesentlicher Bestandteil gesundheitlicher Vorausplanung und damit eine Unterstützung für alle an der Entscheidungsfindung Beteiligten sein. Von diesen Vorsorgemöglichkeiten wird jedoch häufig nicht oder nicht in wirksamer Weise Gebrauch gemacht (In der Schmitten et al. 2014). So fehlt es beispielsweise vielfach an einer ausreichenden Informa-

\footnotetext{
${ }^{1}$ Aus Gründen der Lesbarkeit des Textes wurde die männliche Form gewählt wurde, dennoch bezieht sich die vorliegende Arbeit auf beide Geschlechter.
} 
tion und Aufklärung über die Behandlungsalternativen, so dass dokumentierter und tatsächlicher Wille des Patienten nicht immer übereinstimmen (Becker et al. 2010; Nauck et al. 2014; Schöffner et al. 2012).

Um hier Abhilfe zu schaffen, gibt es in Deutschland heute zahlreiche Beratungsangebote zu den Vorsorgedokumenten. Verbindliche Standards für die Beratung oder die Qualifizierung der Berater bestehen jedoch nicht. Über Struktur und Inhalt der Beratungen ist, ebenso wie über die Wünsche der Berater an die Weiterentwicklung der Vorausplanung, wenig bekannt. In dieser Arbeit werden daher am Beispiel ausgewählter Anbieter von Beratungen zur Patientenverfügung Inhalte und Strukturen der Beratung herausgearbeitet sowie Wünsche der Berater im Hinblick auf die Beratung ermittelt. Darauf aufbauend werden, auch mit Blick auf etablierte umfassende Advance Care Planning Programme (ACP), Perspektiven für eine Weiterentwicklung der Beratung zur Vorausplanung aufgezeigt. Die Arbeit will damit einen Beitrag zur weiteren Verbesserung der Vorausplanung und somit der Entscheidungen am Lebensende leisten.

\subsection{Hintergrund}

Die meisten Menschen sterben heute nicht mehr plötzlich und unerwartet, sondern im hohem Alter nach einer längeren Phase der Multimorbidität (Davies und Higginson 2004; Schildmann et al. 2014). Aufgrund der enorm gestiegenen medizinischen Möglichkeiten werden immer mehr Entscheidungen für oder gegen eine Therapieoption erforderlich. Wie die EURELD-Studie nach der Auswertung von 30.000 Todesfallformularen gezeigt hat, waren $2 / 3$ der Todesfälle vorhersehbar. In bis zu $50 \%$ der Fälle wurde eine Entscheidung gegen eine weitere Therapie gefält (Bosshard et al. 2005; van Delden et al. 2006). Auf Intensivstationen oder in der palliativen Versorgung werden bei bis zu $72 \%$ der dort versterbenden Menschen Entscheidungen zur Therapiezieländerung erforderlich (Sprung et al. 2003; Vincent et al. 2004). Ist schon die Entscheidungsfindung bei einwilligungsfähigen Menschen für alle daran Beteiligten häufig schwierig und belastend, so wird sie umso herausfordernder, wenn der Patient zum Zeitpunkt der Therapieentscheidung nicht einwilligungsfähig ist und sich nicht 
selbst an der Entscheidungsfindung beteiligen kann (Kuehlmeyer et al. 2012; Vig et al. 2007). Die erforderliche Einwilligungsfähigkeit ist bei 48$70 \%$ der Patienten zu dem Zeitpunkt, in dem die Entscheidung getroffen werden muss, nicht vorhanden, der Patient kann seinem Willen nicht unmittelbar rechtsverbindlich Ausdruck verleihen (Silveira et al. 2010; van der Heide et al. 2003). Die Berücksichtigung des Willens des Patienten ist jedoch Voraussetzung für eine rechtmäßige und ethisch gut begründete Therapieentscheidung ( $\S \S 630 d, 1901 a$ I BGB). Es besteht somit ein groBer Bedarf an antizipierenden ${ }^{2}$ oder stellvertretenden Entscheidungen.

Bei vorliegender Einwilligungsunfähigkeit ist daher ${ }^{3} z u$ prüfen, inwieweit der Patient seinem Recht auf Selbstbestimmung durch Nutzung der Vorsorgemöglichkeiten Ausdruck verliehen hat (§§ 1901a, 630d BGB).

Die gesetzlich geregelten Vorsorgemöglichkeiten bestehen aus Patientenverfügung, Vorsorgevollmacht und Betreuungsverfügung. Durch eine Patientenverfügung kann ein einwilligungsfähiger Volljähriger für den Fall seiner Einwilligungsunfähigkeit antizipierend festlegen, welchen medizinischen Maßnahmen er in bestimmten Situationen zustimmt, und welche er ablehnt (§ 1901a I 1 BGB). Mit Hilfe einer Vorsorgevollmacht kann diejenige Person unmittelbar rechtsverbindlich legitimiert werden, die den Willen des Patienten ermitteln und inm zur Umsetzung verhelfen soll (§ 1901a I BGB). Die Erstellung einer Betreuungsverfügung ermöglicht es dem Betreuungsgericht eine Person zu benennen, die die vorgenannten Aufgaben wahrnehmen soll (§§ 1897 I, IV BGB).

Das Bedürfnis nach Wahrung der Selbstbestimmung und die Bereitschaft mit Hilfe der genannten Instrumente Vorsorge zu treffen, ist in der Bevölkerung in den letzten Jahren deutlich gestiegen, wie bereits folgende Zahlen sichtbar machen:

\footnotetext{
${ }^{2}$ Ist genau die Situation eingetreten, die der Verfasser in der Patientenverfügung beschrieben hat, so gilt seine Willensäußerung unmittelbar, d.h. es bedarf keiner weiteren Entscheidung des Vertreters. Dieser hat jedoch die Aufgabe, dem Willen des Patienten zur Umsetzung zu verhelfen (BT-Drucks. 16/8442 S.14, BGH, XII ZB 202/13)

${ }^{3}$ sofern keine mündlich geäußerten Behandlungswünsche für die eingetretene Situation zu ermitteln sind,
} 
Von der Möglichkeit durch eine Patientenverfügung Behandlungswünsche niederzulegen machten im Jahr 2012 ca. 5-42\% der Bevölkerung in Deutschland Gebrauch (Forschungsgruppe Wahlen 2012). Die Prävalenz von Patientenverfügungen steigt danach mit zunehmendem Lebensalter und liegt mit $42 \%$ bei den über 60 -jährigen am höchsten (Forschungsgruppe Wahlen 2012). Im Zentralen Vorsorgeregister der Bundesnotarkammer wurden allein im Jahr 2013423.376 neue Vorsorgedokumente registriert, davon 318.154 in Verbindung mit einer Patientenverfügung (Bundesnotarkammer 2013). Dies bedeutet im Vergleich zum Vorjahr eine Steigerung der Zahl der Eintragungen um 20\%.

In der Praxis zeigt sich jedoch, dass diese Vorsorgemöglichkeiten häufig nicht oder nicht in tragfähiger Weise genutzt werden:

- Die derzeitige Prävalenz von Patientenverfügungen, Vorsorgevollmachten und/oder Betreuungsverfügungen entspricht dem geschilderten hohen Bedarf an antizipierten oder stellvertretenden Entscheidungen nicht.

- Auch wenn eine Patientenverfügung vorliegt, ist die Gefahr, dass diese die konkrete Situation nicht umfasst oder nicht aussagekräftig ist, hoch (Fagerlin und Schneider 2004; Gordy und Klein 2011; Nauck et al. 2014; Sommer et al. 2012).

- Häufig fehlt aufgrund fehlender Information bzw. Aufklärung durch medizinisch geschulte Berater die Kenntnis des Verfügenden über die Implikationen der dokumentierten Wünsche (Schöffner et al. 2012).

- Vielfach besteht eine Inkongruenz zwischen niedergelegtem und tatsächlichem Willen (Nauck et al. 2014; Silvester 2013b)

- In Notfallsituationen sind Patientenverfügungen oft nicht anwendbar. So ist beispielsweise eine mehrseitige Patientenverfügung für den Rettungsdienst in einer eilbedürftigen Akutsituation schon aufgrund ihres Umfanges oft nicht überprüfbar und daher nicht anwendbar (Brokmann et al. 2014). Die indizierten Maßnahmen müs- 
sen somit zunächst eingeleitet werden $^{4}$ (Bienwald et al. 2011; Brokmann et al. 2014; Knittel 2012; Wiese et al. 2011a). Auch sind viele Festlegungen in Patientenverfügungen prognoseabhängig („keine Aussicht auf Besserung“). Eine schnelle Erstellung einer fundierten Prognose ist im Notfall jedoch nicht immer möglich, so dass - in Abwesenheit eines verlässlichen Notfallplans - ebenfalls mit der indizierten Maßnahme begonnen werden muss.

- Ein weiteres Problem liegt in der fehlenden Auffindbarkeit der Patientenverfügungen in der akuten Situation oder bei Verlegungen innerhalb oder zwischen Institutionen (Brokmann et al. 2014; Weinick et al. 2008).

- Viele Menschen verfassen zwar keine Patientenverfügung, benennen aber einen Bevollmächtigten oder Betreuer. So wurden 2013 beim Vorsorgeregister der Notarkammer zwar 423.376 neue Vorsorgedokumente registriert, aber nur 318.154 Verweise (75\%) auf eine zugehörige Patientenverfügung (Bundesnotarkammer 2013). Unbekannt ist, inwieweit im Zusammenhang mit den 25\% der Bevollmächtigungen/Betreuungsverfügungen ohne schriftliche Patientenverfügungen tatsächlich Gespräche über die zukünftigen Behandlungswünsche zwischen Vollmachtgeber und Stellvertreter stattgefunden haben.

- Häufig hat der Stellvertreter wenig oder gar kein Wissen über die Wünsche des Patienten (Nauck et al. 2014). Da der zukünftige Stellvertreter bei der Erstellung der Vollmacht nicht beteiligt werden muss, reicht das Ausfüllen eines z.B. aus dem Internet heruntergeladenen Formulars und dessen Zugang beim Bevollmächtigten für die rechtswirksame Bevollmächtigung aus (Lipp 2009). Nach Aussagen in der Literatur ist zu beobachten, wie in den Krankenhäusern kurz vor Eingriffen oder anderen Maßnahmen unter Einschaltung des Sozialdienstes noch schnell eine Vorsorgevollmacht ausgefüllt wird (Dodegge 2008). Diese Praxis kann dazu führen, dass u.U. ein Mensch Entscheidungen für einen anderen Menschen tref-

\footnotetext{
${ }^{4}$ Sofern sie dem mutmaßlichen Willen des Patienten entsprechen ( $\$ 630 \mathrm{~d}$ I 4 $B G B)$.
} 
fen soll, ohne vorher Gespräche mit inm darüber geführt zu haben. Auch stellt sich die Frage, ob in diesen Fällen bei Vertretenem und Vertreter Klarheit über die mit der Stellvertretung verbundenen Rechte und Pflichten besteht. Wenn Angehörige selbst das Vollmachtformular besorgen und es unterschreiben lassen, besteht diese Gefahr ebenfalls.

- Dem Stellvertreter kommt gemäß § 1901a II BGB die Aufgabe zu, den geäußerten oder mutmaßlichen Willen des Patienten zu ermitteln und umzusetzen (Bienwald et al. 2011). Nicht selten ist es fraglich, inwieweit die Entscheidungen des Stellvertreters tatsächlich auf den Wünschen des Vertretenen beruhen und sie auch entsprechend umgesetzt werden (Azoulay et al. 2003; Bravo et al. 2012; Hauke et al. 2011; Kuehlmeyer et al. 2012; Shalowitz et al. 2006).

- Im Falle der Einsetzung von Berufsbetreuern kennen diese den Patienten und seine Wünsche in der Regel nicht. Die Ermittlung der Behandlungswünsche ist umso schwieriger, wenn keine Patientenverfügung vorliegt.

Somit lässt sich feststellen, dass wesentliche Instrumente zu einer gesundheitlichen Vorausplanung zwar vorhanden sind, aber häufig nicht oder nur fragmentarisch genutzt oder unwirksam gestaltet werden.

Eine fehlende oder nicht tragfähige Vorsorge kann Auswirkungen auf unterschiedlichen Ebenen haben:

- Der Verfasser der Vorsorgedokumente lebt möglicherweise mit der falschen Vorstellung von der Verlässlichkeit der Sicherung seines Selbstbestimmungsrechtes durch die Vorausverfügungen.

- Der Patient trägt das Risiko einer Über- oder Untertherapie, wenn seine Wünsche nicht bekannt sind. Seine Lebensumstände und qualität werden hierdurch nachhaltig beeinflusst. Beispiele hierfür sind die zahlreichen Notfalleinweisungen von hochbetagten, multimorbiden, eventuell auch demenziell veränderten Altenheimbewohnern in ein Krankenhaus (Drehtüreffekt) oder Über- oder Untertherapien bei onkologischen Patienten. 
- Für die Stellvertreter, die Angehörigen, aber auch die Behandelnden, stellt die Entscheidungsfindung, insbesondere von Entscheidungen zur Therapiebegrenzung am Lebensende eine erhebliche psychische Belastung dar (Tilden et al. 2001; Vig et al. 2007; Wendler et al 2011). Diese Belastung scheint umso größer, je unklarer die Wünsche des Betroffenen sind und je weiter sie von den eigenen Einstellungen abweichen (Meeker und Jezewski 2005).

- Für die Organisationen Alten-, Pflegeheim oder Krankenhaus birgt eine unzureichende Vorausplanung neben einem Haftungsrisiko (Palandt et al. 2014) auch die Gefahr, in den Ruf zu geraten, die Wünsche der Bewohner/Patienten nicht wertzuschätzen. Dieses wirkt sich sowohl auf die Zufriedenheit der Bewohner/Patienten, als auch der Angehörigen aus (Ernst et al. 2006).

- Nicht um die Wünsche des Patienten zu wissen kann zu komplexen ethischen Fragestellungen bei der Entscheidungsfindung insbesondere am Lebensende führen5.

- Für das Gesundheitssystem führt eine von den Patienten nicht gewünschte Über- oder Untertherapie oder die Versorgung an einem nicht gewünschten Ort (z.B. Krankenhaus statt Altersheim) zur Fehlallokation von finanziellen und personellen Ressourcen. Auch dieser Faktor ist angesichts der hohen Ausgaben, die für die medizinische Versorgung von Menschen im letzten Lebensjahr aufgewendet werden, nicht außer acht zu lassen (Noethen 2011).

Wesentlicher Einflussfaktor auf die Gestaltung einer tragfähigen gesundheitlichen Vorausplanung ist die Durchführung und Qualität einer Beratung bei der Ermittlung und schriftlichen Niederlegung der Wünsche des Ratsuchenden (Marckmann und In der Schmitten 2013; Nauck et al. 2014; Schöffner et al. 2012). Sie kann der Schlüssel für eine aussagekräftige, anwendungstaugliche Dokumentation informierter Patientenwünsche, die

\footnotetext{
${ }^{5}$ Darauf lässt z.B. die steigende Zahl an Leitlinien zur Therapiezielbestimmung schließen, die als Hilfestellung zur Lösung dieser Konflikte konzipiert wurden (Simon und May) . Die Ermittlung und Berücksichtigung (ggf. mutmaßlichen) Patientenwillens ist Voraussetzung der ethisch und rechtlich tragfähigen Therapiezielfestlegung (§§ 630d, 1901a BGB).
} 
rechtzeitige Benennung und Einbeziehung des zukünftigen Vertreters, sowie für klare Absprachen über die Auffindbarkeit der Dokumente sein. Eine Beratung zur Patientenverfügung ist in Deutschland nicht gesetzlich vorgeschrieben. Dennoch gibt es, neben den Ärzten, eine schwer zu überblickende Vielzahl von Berufs- und Personengruppen, die sich für die Verbesserung der gesundheitlichen Vorausplanung - hier hauptsächlich in der Beratung zu den Vorsorgedokumenten - engagieren. Beispielhaft sind in diesem Zusammenhang Betreuungsvereine, Rechtsanwälte und Notare, Hospizvereine, unabhängige Patientenberatungen, Sozialarbeiter, Seelsorger und Krankenkassen zu nennen. Seit Jahren wird stetig an der Weiterentwicklung der Vorsorgedokumente, des Informationsmaterials und der Öffentlichkeitsarbeit gearbeitet. Daneben wird nach Möglichkeiten der Verbesserung der individuellen Beratung gesucht. Es besteht das Bewusstsein, dass die Förderung gesundheitlicher Vorausplanung alleine durch eine legalistische und isolierte Beratung beim Ausfüllen der Vorsorgeformulare unzureichend ist. Zunehmend wird daher eine systematische, multidisziplinäre und prozesshafte Begleitung gefordert (In der Schmitten 2013; May et al. 2005; Simon 2010; Wiese et al. 2008). Erfolgreiche Beispiele hierfür finden sich in anderen westlichen Ländern, die eine ähnliche Situation bei der Vorausplanung haben. So wurden seit Anfang der 1990er Jahre in den USA umfassende Programme zur vorausschauenden Gesundheitsplanung entwickelt und unter der Bezeichnung "Advance Care Planning" (ACP) international erfolgreich. Diese Programme beinhalten neben professionell begleiteten, strukturierten und umfassenden $\mathrm{Ge}$ sprächsprozessen auch eine regionale Implementierung von Standards zur Erfassung und Umsetzung des Patientenwillens (Hammes et al. 2010; In der Schmitten et al. 2014; Silvester et al. 2013b).

In Deutschland gibt es keine verbindlichen Standards oder einheitlichen Konzepte für den Beratungsablauf und -inhalt oder die Qualifikation der Berater. Jeder Berater und jede Organisation kann die Beratung nach eigenen (Wert-) Vorstellungen anbieten und gestalten. Es ist wenig über Struktur und Inhalt der Beratungspraxis zur Vorausplanung der vielen verschiedenen Anbieter und damit der Ausgangsbasis für Weiterentwicklungen der Vorausplanung bekannt. 


\subsection{Zielsetzung}

Ziel der vorliegenden Arbeit ist es, durch eine Befragung von Beratern einen Einblick in die aktuelle Beratungssituation zur Patientenverfügung in einer bestimmten Region zu erhalten und darauf aufbauend Hinweise für die Weiterentwicklung der Beratung abzuleiten. Die Beurteilung der empirischen Befunde soll auch den Blick auf die best practice erfolgreicher, umfassender ACP-Programme einbeziehen. Weiterhin soll die vorliegende Arbeit eine empirische Grundlage für zukünftige Studien zur gesundheitlichen Vorausplanung bieten.

\subsection{Fragestellung}

Um das genannte Ziel der Studie zu erreichen, sollen folgende Fragen bearbeitet werden:

- Welche Struktur und welche Inhalte haben Beratungsgespräche zur Patientenverfügung?

- Wie unterscheiden sich diese in Abhängigkeit von der Beratergruppe?

- Gibt es klare Beratungsprofile der verschiedenen Beratergruppen?

- Wie ist das Umfeld der Beratung, die Vernetzung der Beratung?

- Welche Wünsche haben die Berater an die Weiterentwicklung der Beratung?

- Welche Hinweise für die Weiterentwicklung der Beratung können, auch im Hinblick auf international etablierte ACP Programme aus den gewonnenen Daten abgeleitet werden? 


\section{Methode}

Die vorliegende Untersuchung erfolgte, da es sich bei der Beratung zur Patientenverfügung um aktuelle soziale Prozesse handelt, in der Form einer empirischen Studie (Bortz und Döring 2006). Die Erhebung von Daten mittels eines Fragebogens erlaubte es, systematisch Informationen über die Beratungssituation zu sammeln und darauf aufbauend Arbeitshypothesen für die Weiterentwicklung der Beratung zu erstellen. Die Studie hat explorativ-deskriptiven Charakter, da bisher noch kaum Daten zu Inhalt und Struktur der Beratung sowie den Wünschen der Berater in Deutschland vorliegen.

\subsection{Planung und Vorbereitung der Datenerhebung}

\subsubsection{Entwicklung des Forschungsinstrumentes}

Zur Bearbeitung der Forschungsfragen konnte kein geeignetes bereits existierendes Forschungsinstrument gefunden werden. Daher wurde ein Online-Fragebogen entwickelt, der sich für alle Beratergruppen in gleicher Form eignete. Der quantitative Ansatz wurde gewählt, um die Befragung relativ vieler Berater mit demselben Erhebungsinstrument zu ermöglichen. So sollte eine bessere Vergleichbarkeit der Antworten, und die Option zur Beschreibung von Beratungen in verschiedenen Settings geschaffen werden. Ferner konnten mit dieser Methode Einflussnahmen auf das Antwortverhalten durch einen Interviewer ausgeschlossen werden. Der Fragebogen wurde mit dem Programm „Unipark“ Version EFS 10.1 entwickelt.

Die Datenerhebung erfolgte anonym, um eine Rückführung der Antworten auf die teilnehmenden Personen zu verhindern und so die Offenheit und Ehrlichkeit bei den Antworten zu erhöhen.

Der Fragebogen enthielt überwiegend Fragen, die im Rahmen einer Likert Skala mit Ordinalskala beantwortet werden sollten. Um ein Ausweichen auf eine mittlere Stufe zu unterbinden und eine Entscheidung für eine klare Aussage zu provozieren, wurde die Skala vierstufig erstellt. 
Zur Erleichterung einer zügigen Bearbeitung des Fragebogens waren sieben Fragen in einer ja/nein Dichotomie zu beantworten. Fünf Fragen wurden als offene Fragen, bei denen die Befragten die Antworten selber formulieren mussten, gestaltet, damit in diesen Bereichen eine Einflussnahme auf die Aussagen oder eine Einengung des Antwortspektrums durch vorformulierte Antworten ausgeschlossenen werden konnte. Acht Fragen enthielten neben einem Antwortkatalog auch ein offenes Feld, in dem freie Angaben gemacht werden konnten.

Inhaltlich gliederte sich der Fragebogen in vier Bereiche: Teil A „Zur Beratungstätigkeit" erfragte Struktur und Inhalt des Beratungsgespräches. Teil B befasst sich mit dem „Umfeld der Beratung“, insbesondere mit der Vernetzung der Berater und ihren Wünschen an die Weiterentwicklung der Beratung. In Teil C wurden Daten zur „Person des Beraters“, zu seiner Ausbildung und Vergütung erhoben.

Um auch den Personen, die zum Adressatenkreis des Fragebogens gehören, aber zur Zeit nicht aktiv beraten, die Möglichkeit zur Äußerung zu den Gründen dafür zu geben, enthielt der Fragebogen eine Weiche. Diese Weiche führte die Personen, die die erste Frage der Fragebogens „Beraten Sie zur Patientenverfügung?“ verneinen, direkt auf die „Nicht-Berater Auskunft" (6.1).

In den an die Ärzte adressierten Online Fragebogen wurde, neben der Frage nach der hausärztlichen Tätigkeit (A1), die Frage nach der Postleitzahl aufgenommen. So konnten bei der Datenbereinigung Teilnehmer ausgefiltert werden, die außerhalb der beruflichen oder regionalen Eingrenzung der Umfrage tätig waren.

Bezüglich der Art der Versendung und Bearbeitung des Fragebogens (Online/Papier) ist mit Vertretern der verschiedenen Adressatenkreise gesprochen worden. Die Vertreter der Ärzte sprachen sich für einen Faxversand aus. Alle anderen Gruppen favorisierten aufgrund der schnelleren Bearbeitbarkeit eine Zusendung per Email als Online Fragebogen („Papier landet gleich im Papierkorb“).

Der Fragebogen wurde daher als Online Fragebogen gestaltet, um möglichst hohe Rückläufe auch bei den Nicht-Ärzten zu fördern, und so vielfältigere Aussagen zu erhalten. Weitere Argumente für die Wahl des Online 
Formates waren die geringere Anfälligkeit für Übertragungsfehler bei der Auswertung und die schnellere Bearbeitbarkeit der Daten.

\subsubsection{Pretest}

Die Grundkonzeption des Fragebogens wurde 34 Teilnehmern einer Schulung zur Beratung zur Patientenverfügung der Hospizakademie Nürnberg zur Testung vorgelegt. Ein beigefügter Evaluationsbogen ermöglichte ein Feedback zum Fragebogen.

\begin{tabular}{|l|c|c|c|l|}
\hline & ja & nein & $\begin{array}{c}\text { keine } \\
\text { Angabe }\end{array}$ & $\begin{array}{l}\text { Bemer- } \\
\text { kungen }\end{array}$ \\
\hline Waren die Fragen gut verständlich? & 27 & 1 & 6 & \\
\hline $\begin{array}{l}\text { Falls nein, welche Fragen waren nicht so gut } \\
\text { verständlich? }\end{array}$ & 1 & 0 & 33 & $\begin{array}{l}\text { Fragen } \\
7,8(1 \mathrm{TN})\end{array}$ \\
\hline War die Anzahl der Fragen passend? & 27 & 0 & 7 & \\
\hline Haben Sie Fragen vermisst? & 0 & 28 & 6 & \\
\hline Wenn ja, welche? & 0 & 0 & 34 & \\
\hline Waren die Antwortkategorien passend? & 25 & 2 & 7 & \\
\hline
\end{tabular}

Tabelle 1: Pretest mit 34 Teilnehmern einer Schulung zur Beratung zur Patientenverfügung

Die Angaben zur benötigten Zeit zum Ausfüllen: 23 Teilnehmer gaben zwischen 8-15 Minuten, 1 Teilnehmer 25 Minuten, 2 Teilnehmer weniger als 6 Minuten, 1 Teilnehmer lediglich „kurz" als benötigte Zeit an und ein Teilnehmer macht keine Angabe. Daher wurde davon ausgegangen, dass die benötigte Dauer für das Ausfüllen des Fragebogens mit ca. 8-15 Minuten für die Teilnehmer zumutbar ist.

Nach einer ersten Überarbeitung wurde der Fragebogen Mitgliedern der verschiedenen Beratergruppen zur Testung nach der Methode „Think aloud“ gegeben. Diese Methode kam zur Anwendung, um den Zeitaufwand für die Tester so kurz wie möglich zu halten und eventuell auftretende Fragen sofort klären zu können. Während die Tester den Fragebogen in Papierform ausfüllten, nahm die Verfasserin des Bogens die dabei gemachten Anmerkungen auf.

Im Laufe des Prozesses der Testung wurde der Fragebogen zunächst gestrafft, indem bei etlichen Fragen die Antwortkategorien von einer Likert 
Skala auf eine ja/nein Dichotomie umgestellt wurden. Aufgrund der geringen Varianzen bei den Antworten war in diesen Bereichen kein Erkenntnisverlust durch die Straffung zu befürchten. Zur Vermeidung eines Ausweichens auf einen neutralen Mittelwert wurde die Likert Skala von fünfstufig auf vierstufig verkürzt. Weiterhin wurden allgemeinere Fragen gestrichen, um Raum für detailliertere Fragen zum Gesprächsinhalt (A12), zur Thematik der Organspende (A17,18), zur Vernetzung (B 3-8), sowie zur Person zu gewinnen (C6-9) und so den Erkenntniswert der Untersuchung zu steigern. Die Fragen zur Organspende (A 17,18) wurden auf Anregung von PD. Dr. med., Dr. phil. Ralf Jox, Institut für Ethik, Geschichte und Theorie der Medizin, Ludwig-Maximilians-Universität München eingefügt.

Mit Herrn Dr. Crispin vom Institut für Medizinische Informationsverarbeitung, Biometrie und Epidemiologie der Ludwig-Maximilians-Universität München wurde die Eignung des Fragebogens insbesondere für eine Auswertung durch das Statistikprogramm SPSS diskutiert, und daraufhin die Zahl der offenen Felder reduziert.

Im Anschluss an die Testung wurde der gesamte Fragebogen noch einmal auf seine Verständlichkeit für die verschiedenen Beratergruppen und deren spezifische Blickwinkel und Sprachverständnisse hin (was bedeutet z.B.: „Zuhause“, wie ist das Verständnis von „Beratung“) überprüft und entsprechend angepasst.

Die Überprüfung der Funktionalität des Fragebogens im Online Format auf verschiedenen Rechnern und unter verschiedenen Betriebssystemen erfolgte durch Bekannte und Verwandte der Verfasserin.

\subsubsection{Auswahl der Stichprobe}

Da eine kaum zu überblickende Vielfalt an Menschen und Personengruppen in Deutschland zur Patientenverfügung berät, beschränkt sich die Umfrage regional auf den Raum München und dort auf bestimmte Beratergruppen.

Bei der Auswahl der Teilnehmer lag der Schwerpunkt auf Beratergruppen, die ambulant beraten, weil nicht nur die Beratung bereits erkrankter Menschen (z.B. in Krankenhäusern) erforscht werden sollte. Zu diesen Bera- 
tern gehören traditionell und wesentlich Hausärzte, Notare und Mitarbeiter von Hospizvereinen. Die Überleitungsfachkräfte in Pflegeheimen/vollstationären Pflegeeinrichtungen ${ }^{6}$ wurden einbezogen, da sie an einer wichtigen Schnittstelle zwischen ambulanter und stationärer Versorgung tätig sind und für eine Personengruppe arbeiten, bei der der Bedarf und die Prävalenz an Patientenverfügungen hoch sein sollte (Forschungsgruppe Wahlen 2012). Der Aufgabenbereich der Überleitungsfachkräfte „... beinhaltet sämtliche Interventionen, die bei einem Einzug eines pflegebedürftigen Menschen in eine vollstationäre Pflegeeinrichtung, einem Umzug innerhalb der Einrichtung, sowie in Zusammenhang mit einem Krankenhausaufenthalt Versorgungsbrüche verhindern und die Kontinuität der Pflege sicherstellen“ (Landeshauptstadt 2010). Dieses umfasst auch die Aufgabe, Fragen zum Lebensende wie z.B. Patientenverfügungen oder Wünsche für die Sterbebegleitung anzusprechen. Daher kam auch diese Berufsgruppe als Kreis möglicher Berater in Frage und wurde in die Umfrage einbezogen.

\subsubsection{Ethikkommission}

Die Ethikkommission der Ludwig-Maximilians-Universität München erteilte am 04.06.2013 eine Unbedenklichkeitsbescheinigung für die Studie, da die Datenerhebung anonymisiert erfolgte (UE Nr 091-13).

\subsection{Durchführung der Umfrage}

\subsubsection{Rekrutierung der Teilnehmer}

Die Rekrutierung der Teilnehmer erfolgte auf verschiedenen Wegen.

Bei den Notaren wurde die Studie zunächst dem Obmann der Bezirksgruppe München I im Bayerischen Notarverein vorgestellt. Dieser versandte im Anschluss daran die Email mit der Einladung zur Teilnahme an der Studie und dem Link zum Zugang an alle 79 Notare dieser Bezirks-

\footnotetext{
${ }^{6}$ Erfasst wurden Einrichtungen gem. SGB XI.
} 
gruppe. Das Erinnerungsschreiben konnte ebenfalls über diesen Verteiler in Umlauf gebracht werden.

Ähnlich war das Vorgehen bei den vier Münchner Hospizvereinen: Auch hier ist das Projekt im ersten Schritt den Leitungen vorgestellt worden. Anschließend schickten diese die Einladung an alle 18 haupt- und vier ehrenamtlichen Mitarbeiter, die als Berater in Frage kamen. Später wurde über diesen Weg nochmals an die Umfrage erinnert.

Bei den 54 Pflegeheimen in der Stadt München wurden die Pflegedienstleitungen angeschrieben und gebeten, die Einladungen und später die Erinnerung an die Überleitungsfachkräfte weiterzuleiten.

Schwierig war die Rekrutierung der Ärzte. Weder der Bayerische Hausärzteverband (BHÄV), noch der Ärztliche Kreis- und Bezirksverband (ÄKBV) oder die Bayerische Landesärztekammer verfügten zur Zeit der Umfrage über einen umfassenden und aktuellen Emailverteiler für Hausärzte im Raum München. Daher wurde, nach Rücksprache mit den Vorständen und Geschäftsführerinnen der BHÄV und des ÄKBV, wie folgt vorgegangen: Der ÄKBV versandte ein Fax mit der Einladung zur Teilnahme an der Umfrage und dem entsprechenden Link an alle 2552 niedergelassenen Hausärzte, hausärztliche und fachärztliche Internisten, sowie dort angestellte Ärzte in Stadt und Landkreis München ${ }^{7}$. Zusätzlich wurde ein Aufruf zur Teilnahme in den Münchner Ärztlichen Anzeigen veröffentlicht und ein Link zur Umfrage auf die Startseite des ÄKBV gestellt. Flankierend ist in der Delegiertenversammlung des ÄKBV auf die Umfrage hingewiesen worden.

Der BHÄV unterstützte die Umfrage, indem er in seinem elektronischen Newsletter darauf hinwies ${ }^{8}$.

\footnotetext{
${ }^{7}$ Die fachärztlichen Internisten konnten aus EDV-technischen Gründen nicht ausgegrenzt werden. Sie wurden aber, ebenso wie regional nicht zugehörige Ärzte, im Rahmen der Datenbereinigung über die Filterfragen (s.o.), von der Auswertung für Hausärzte ausgeschlossen.

${ }^{8}$ Dieser Newsletter erreicht nach Schätzung der Geschäftsführerin der BHÄV ca. 300 Ärzte im Raum München.
} 


\subsubsection{Studienverlauf}

Die Umfrage unter Mitarbeitern von Hospizvereinen, Notaren und Überleitungskräften lief vom 16.07.2013 bis 31.10.2013, die unter Ärzten, bedingt durch die schwierige Findung eines Zugangsweges und die Sommerferien, vom 9.10 .2013 bis $6.12 .2013^{9}$.

\subsubsection{Auswertung}

Die Auswertung erfolgte mit Hilfe des Statistikprogrammes SPSS (Version 21) und Excel.

\footnotetext{
${ }^{9}$ Mit Hinblick auf die Sommerferien wurde die Laufzeit bei den Nicht-Ärzten etwas länger als bei den Ärzten angelegt.
} 


\section{Ergebnisse}

Die Darstellung der Ergebnisse folgt im Wesentlichen dem Ablauf des Fragebogens. Einige Aspekte wurden vorgezogen, um die Sinnzusammenhänge besser darstellen zu können. Die zur Person erhobenen Daten finden sich am Anfang der Darstellung. Zur besseren Übersichtlichkeit sind die Prozentzahlen auf ganze Zahlen gerundet.

\subsection{Teilnehmer}

\subsubsection{Stichprobe}

Der Rücklauf war, je nach Gruppe, sehr unterschiedlich. Von 22 befragten Mitarbeitern der Hospizvereine füllten 20 Personen (91\%) den Fragebogen aus. Von 54 befragten Überleitungskräften wurden 21 Bögen bearbeitet $(39 \%)$. Bei den Notaren beteiligten sich 26 (33\%) der eingeladenen 79 Notare.

Die Ärzte klickten den für sie konzipierten Link zur Umfrage 292mal an (Startseite). 220 Nutzer (8,6\%) klickten über die Startseite hinaus weiter und sandten den Fragebogen nach Erreichen der letzten Seite ab; davon sahen allerdings 36 Nutzer den Fragebogen nur durch, ohne Angaben zu machen. Im Rahmen der Datenbereinigung wurden diese Datensätze und Datensätze von nicht in Stadt oder Landkreis München praktizierenden und/oder Nicht-Hausärzten aussortiert. Danach verblieben 131 beratende und 9 nicht-beratende Hausärzte in der Auswertung (140=5,5\%).

Die vorliegende Studie bezieht aus Kapazitätsgründen nur die beratenden Mitglieder der jeweiligen Beratergruppen ein. 
Nach der Datenbereinigung standen somit insgesamt 198 Datensätze von Beratern zur Patientenverfügung zur Auswertung zur Verfügung (Tab.2).

\begin{tabular}{|l|c|c|}
\hline Beratergruppe & Personen & \% \\
\hline Hausärzte & 131 & $66 \%$ \\
\hline Notare & 26 & $13 \%$ \\
\hline Überleitungsfachkräfte & 21 & $11 \%$ \\
\hline Mitarbeiter Hospizvereine & 20 & $10 \%$ \\
\hline gesamt & $\mathbf{1 9 8}$ & $\mathbf{1 0 0 \%}$ \\
\hline
\end{tabular}

Tabelle 2: Funktion der teilnehmenden Berater

\subsubsection{Alter der Teilnehmer}

Das durchschnittliche Alter der Teilnehmer in den jeweiligen Gruppen war mit einem Mittelwert zwischen 50 bis 54 Jahren sehr ähnlich (Tab.3).

\begin{tabular}{|l|c|c|c|c|}
\hline & $\begin{array}{c}\text { Alter } \\
\text { Median }\end{array}$ & $\begin{array}{c}\text { Alter } \\
\text { Mittelwert }\end{array}$ & $\begin{array}{c}\text { Alter } \\
\text { Minimum }\end{array}$ & $\begin{array}{c}\text { Alter } \\
\text { Maximum }\end{array}$ \\
\hline Hausärzte & 53 & 54 & 32 & 74 \\
\hline Notare & 49 & 52 & 39 & 70 \\
\hline Überleitungsfachkräfte & 54 & 50 & 36 & 63 \\
\hline Mitarbeiter Hospizvereine & 53 & 53 & 36 & 86 \\
\hline
\end{tabular}

Tabelle 3: durchschnittliches Alter der Teilnehmer

\subsubsection{Verteilung der Geschlechter}

Die teilnehmenden Mitarbeiter der Hospizvereine waren ca. zu drei Viertel weiblich. Auch bei den Überleitungsfachkräften überwogen die weiblichen Teilnehmer (Tab.4). 


\begin{tabular}{|l|c|c|c|c|}
\hline & männlich & weiblich & $\begin{array}{c}\text { keine } \\
\text { Angabe }\end{array}$ & gesamt \\
\hline Hausärzte & $49 \%$ & $44 \%$ & $8 \%$ & $100 \%$ \\
\hline Notare & $81 \%$ & $19 \%$ & $0 \%$ & $100 \%$ \\
\hline Überleitungsfachkräfte & $10 \%$ & $62 \%$ & $29 \%$ & $100 \%$ \\
\hline Mitarbeiter Hospizvereine & $25 \%$ & $70 \%$ & $5 \%$ & $100 \%$ \\
\hline
\end{tabular}

Tabelle 4: Geschlechterverteilung

Ein anderes Bild zeigte sich bei den befragten Notaren: Hier waren weniger als ein Fünftel der Teilnehmer weiblich. Bei den Hausärzten war die Verteilung zwischen weiblichen und männlichen Teilnehmern relativ gleichmäßig.

\subsubsection{Jahre der ausgeübten Beratertätigkeit}

Die Beratungserfahrung der Teilnehmer der Studie war sehr unterschiedlich (Tab.5).

\begin{tabular}{|l|c|c|c|c|}
\hline & $\begin{array}{c}\text { Jahre } \\
\text { Erfahrung } \\
\text { Median }\end{array}$ & $\begin{array}{c}\text { Jahre } \\
\text { Erfahrung } \\
\text { Mittelwert }\end{array}$ & $\begin{array}{c}\text { Jahre } \\
\text { Erfahrung } \\
\text { Minimum }\end{array}$ & $\begin{array}{c}\text { Jahre } \\
\text { Erfahrung } \\
\text { Maximum }\end{array}$ \\
\hline Hausärzte & 10 & 13 & 0 & 40 \\
\hline Notare & 16 & 20 & 6 & 40 \\
\hline Überleitungsfachkräfte & 11 & 11 & 0 & 25 \\
\hline Mitarbeiter Hospizvereine & 9 & 9 & 0 & 23 \\
\hline
\end{tabular}

Tabelle 5: Jahre der Beratungstätigkeit

Die Notare stellten die Gruppe mit der längsten Beratungserfahrung. Bemerkenswert ist hier, dass alle teilnehmenden Notare mindestens sechs Jahre Beratungserfahrung angaben. Danach folgten, allerdings mit großem Abstand, die Überleitungsfachkräfte und die Mitarbeiter der Hospizvereine. 


\subsubsection{Anzahl der Einzelberatungen}

Auch die Anzahl der im Jahr 2012 durchgeführten Beratungen war sehr unterschiedlich (Tab.6).

\begin{tabular}{|l|c|c|c|c|}
\hline & $\begin{array}{c}\text { Gespräche } \\
\text { Median }\end{array}$ & $\begin{array}{c}\text { Gespräche } \\
\text { Mittelwert }\end{array}$ & $\begin{array}{c}\text { Gespräche } \\
\text { Minimum }\end{array}$ & $\begin{array}{c}\text { Gespräche } \\
\text { Maximum }\end{array}$ \\
\hline Hausärzte & 10 & 16 & 0 & 150 \\
\hline Notare & 50 & 61 & 20 & 120 \\
\hline Überleitungsfachkräfte & 25 & 46 & 0 & 250 \\
\hline Mitarbeiter Hospizvereine & 35 & 56 & 0 & 200 \\
\hline
\end{tabular}

Tabelle 6: Anzahl der Einzelgespräche in 2012

Bemerkenswert an diesen Ergebnissen ist zunächst die weite Spanne der Anzahl der Beratungen innerhalb und zwischen den Gruppen (0-250 Beratungen). Die Notare stellten die Gruppe mit den meisten Beratungen pro Berater im Jahr 2012. Die Mitarbeiter der Hospizvereine und die Überleitungsfachkräfte berieten mit 35 bzw. 25 Beratungen pro Person (Median) deutlich weniger.

Weitaus weniger häufig waren die Ärzte in diesem Bereich tätig: Geht man von 10 Beratungen pro Arzt aus (Median) und unterstellt eine regelmäßige Verteilung über das Jahr, so wurde pro Monat weniger als eine Beratung durchgeführt.

\subsection{Inhalt und Struktur der Beratungstätigkeit}

\subsubsection{Veranlasser der Beratung}

Die Teilnehmer der Studie benannten weit überwiegend die Ratsuchenden (d.h. die beratenen Personen) als diejenigen, die häufig (95 Nennungen) oder fast immer (70 Nennungen) die Beratung veranlassten (Abb.1). 
Abbildung 1: Alle Berater $(n=198)$ : Hauptveranlasser der Beratung

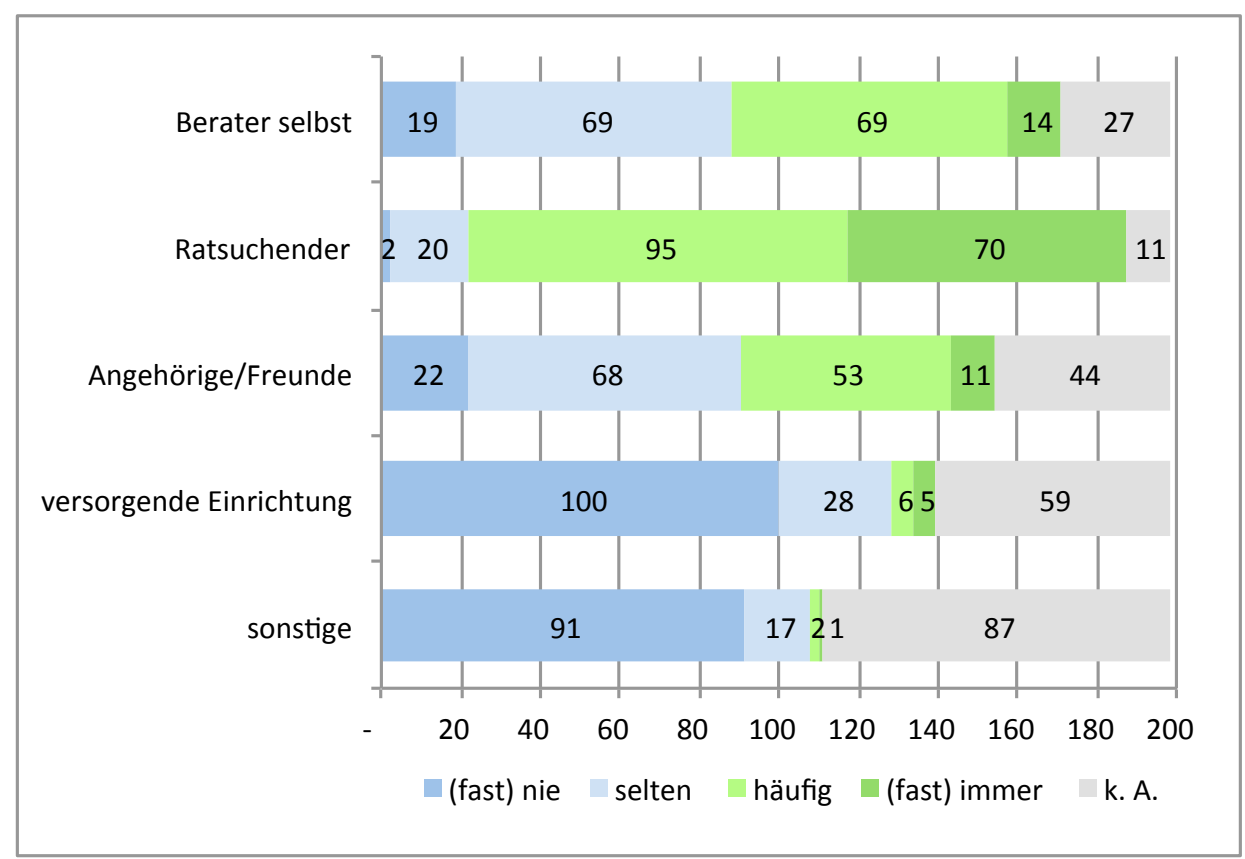

An zweiter Stelle folgten die Berater selbst, an dritter Stelle Angehörige und Freunde. Versorgende Einrichtungen und sonstige spielten bei der Initiation der Beratung fast keine Rolle.

Differenziert man nach Beratergruppen sieht man folgende Unterschiede: 
Abbildung 2: Veranlasser der Beratung

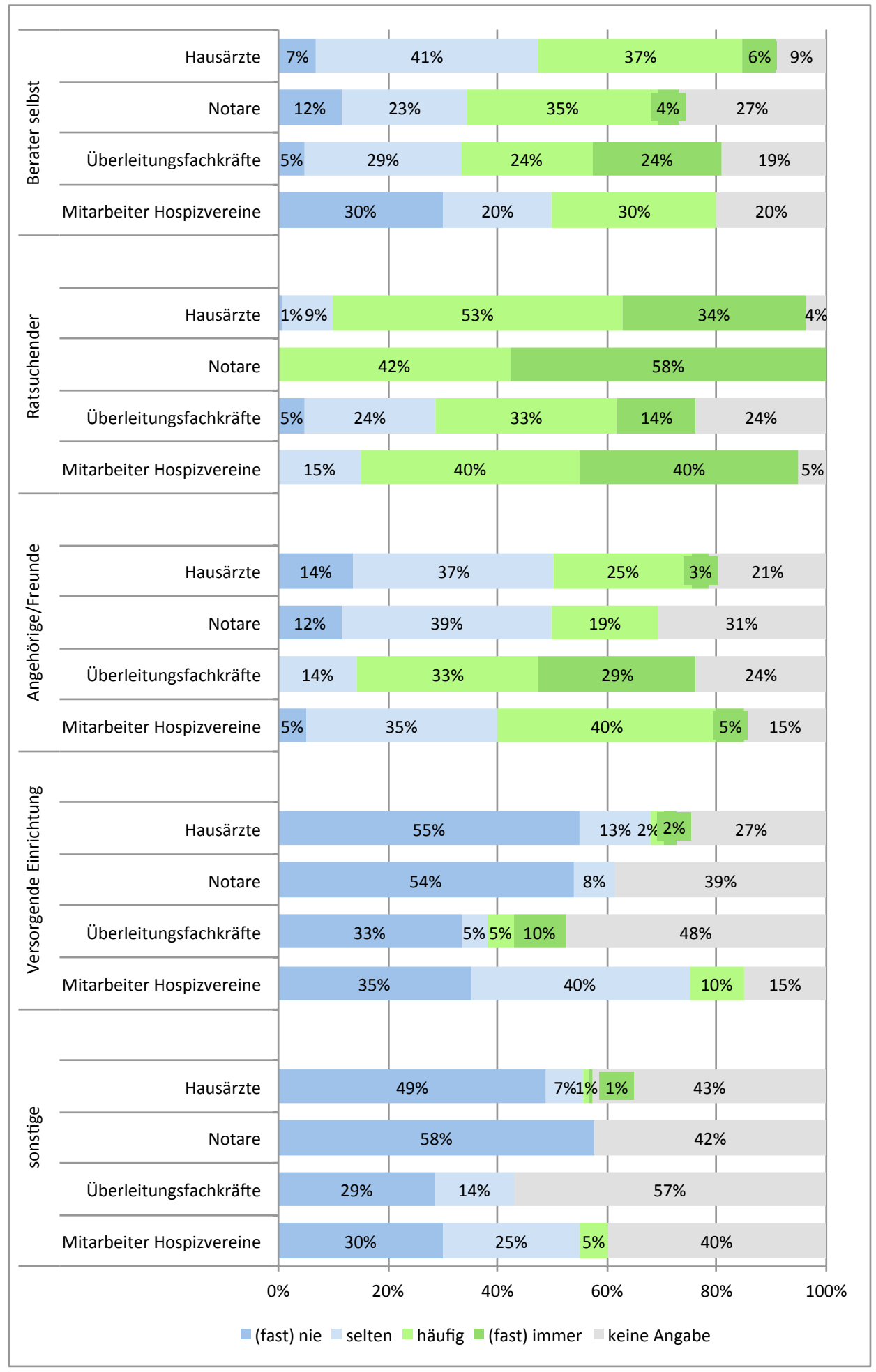


Die Ärzte gaben weit überwiegend die Ratsuchenden als Hauptveranlasser der Beratung an (häufig (h)/(fast)immer (i) 87\%). Bei der Angabe der Häufigkeit der Veranlassung durch die Ärzte selbst zeichneten sich zwei Gruppen ab: $48 \%$ sahen sich selten (s) oder fast nie (n) in der Rolle der Initiatoren, $43 \%$ dagegen häufig (h) oder fast immer (i). Angehörige oder Freunde des Ratsuchenden wurden von gut einem Viertel der befragten Ärzte als Veranlasser angegeben (h/i 28\%). Versorgenden Einrichtungen und "Sonstigen“ kam im Bereich der Hausärzte eine weit nachrangige Relevanz zu (h/i 4\%).

Bei den Notaren stellten die Ratsuchenden ebenfalls die mit großem Abstand größte Gruppe der Veranlasser einer Beratung dar (h/i 100\%). Eine bemerkenswerte Rolle bei der Initiierung der Gespräche kam den Notaren selbst zu: Über ein Drittel der Teilnehmer aus dieser Berufsgruppe gab an, selbst häufig oder fast immer die Beratung zu veranlassen (h/i 39\%). Eine ähnlich große Gruppe erklärte aber auch, fast nie oder selten in diesem Zusammenhang aktiv zu werden (n/s 35\%). Angehörigen und Freunden schrieben sie in diesem Kontext eine deutlich geringere Bedeutung zu als allen anderen Gruppen (h 19\%). Versorgende Einrichtungen oder „Sonstige" hatten fast keinen Einfluss (h/i 15\%).

Aus den Angaben der Überleitungsfachkräfte wurde die starke Bedeutung der Angehörigen/Freunde in diesem Setting deutlich: 62\% der Berater benannten sie als diejenigen, die häufig oder fast immer die Beratung veranlassen. Die zweitgrößte Bedeutung kam den Überleitungsfachkräften selbst (h/i 48\%), aber auch in ähnlichem Umfang den Ratsuchenden zu (h/i 47\%). Die Relevanz der versorgenden Einrichtungen oder „sonstiger“ für die Veranlassung der Beratung war deutlich nachgelagert (h/i 15\%).

Beratungen durch Mitarbeiter von Hospizvereinen veranlassten weit überwiegend die Ratsuchenden selbst (h/i 80\%). Auch Angehörige und Freunde spielten in diesem Zusammenhang eine große Rolle, da sie von $40 \%$ der Berater als häufige Initiatoren und von $5 \%$ der Berater als diejenigen, die fast immer initiieren, angegeben wurden. An dritter Stelle sahen sich die Mitarbeiter der Hospizvereine selbst zu 30\% häufig die Initiative zu einer Beratung ergreifen. Eine nachgeordnete Rolle nahmen versorgende Einrichtungen und „Sonstige“ ein. 
Fasst man die Ergebnisse zur Frage nach der Veranlassung der Beratung zusammen, zeigt sich deutlich, dass die Initiative zu einer Beratung bei diesen Beratergruppen weit überwiegend vom Ratsuchenden selbst ausging. Mit Ausnahme der Überleitungsfachkräfte, die die Angehörigen als Hauptveranlasser benannten (h/i 62\%), gaben alle anderen Gruppen die Ratsuchenden als Hauptinitiatoren an (h/i 80-100\%). Die beratenden Ärzte, Notare und Überleitungskräfte sahen sich selbst an zweiter Stelle der Veranlasser einer Beratung. Angehörige wurden von Ärzten und Notaren als dritthäufigste Gruppe von Veranlassern benannt (h/i $28 \%, 19 \%)$, bei den Überleitungskräften waren sie die Hauptveranlasser, von den Beratern der Hospizvereine wurden sie als zweithäufigste Veranlasser benannt.

Bemerkenswert war, dass versorgende Einrichtungen, auch bei den dort arbeitenden Überleitungsfachkräften, kaum eine Rolle spielten.

\subsubsection{Gesundheitszustand der Ratsuchenden}

Die weitaus meisten Menschen, die eine Beratung durch die Teilnehmer der Studie erhielten, waren nach Kenntnis ihrer Berater gesund (Abb.3).

Abbildung 3: Alle Teilnehmer $(n=198)$ : Gesundheitszustand der meisten Ratsuchenden

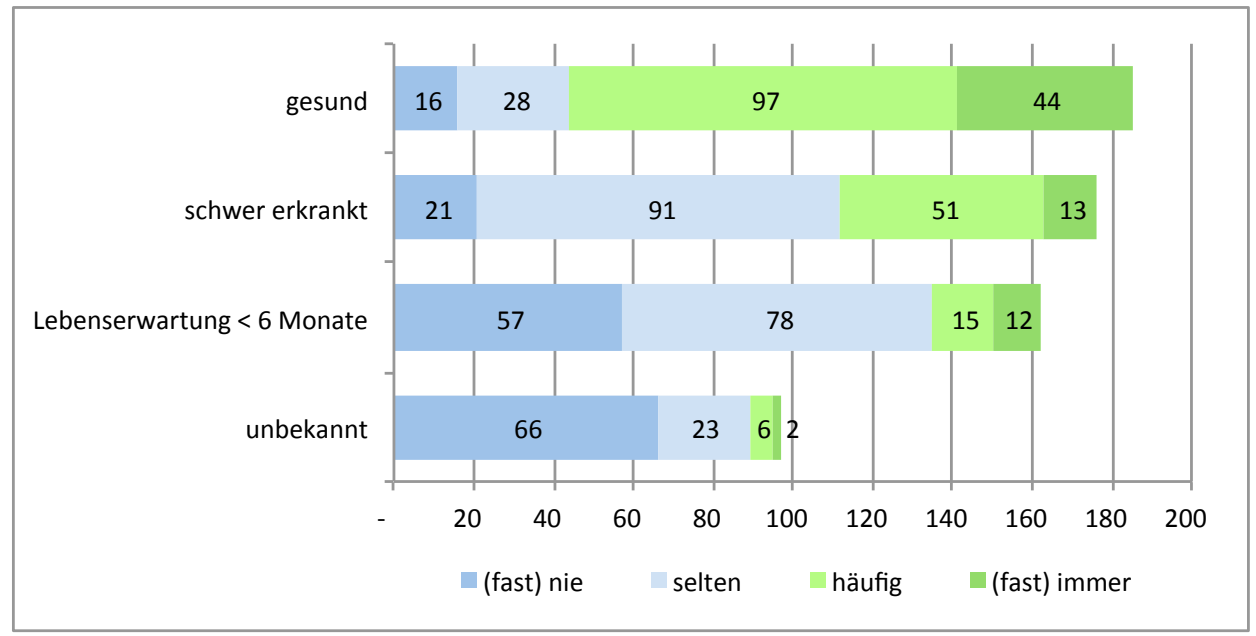


Nur ein Drittel der Teilnehmer berichtete, häufig oder fast immer schwerkranke Menschen zu beraten. Auffällig war, dass die Menschen mit einer Lebenserwartung von unter sechs Monaten die mit großem Abstand kleinste Gruppe der Ratsuchenden bildeten (h/i 27\%). Nicht in allen Fällen war der Gesundheitszustand den Beratern bekannt.

Betrachtet man die Verteilung auf die einzelnen Beratergruppen, so werden folgende Tendenzen sichtbar (Abb.4).

Abbildung 4: Gesundheitszustand der meisten Ratsuchenden

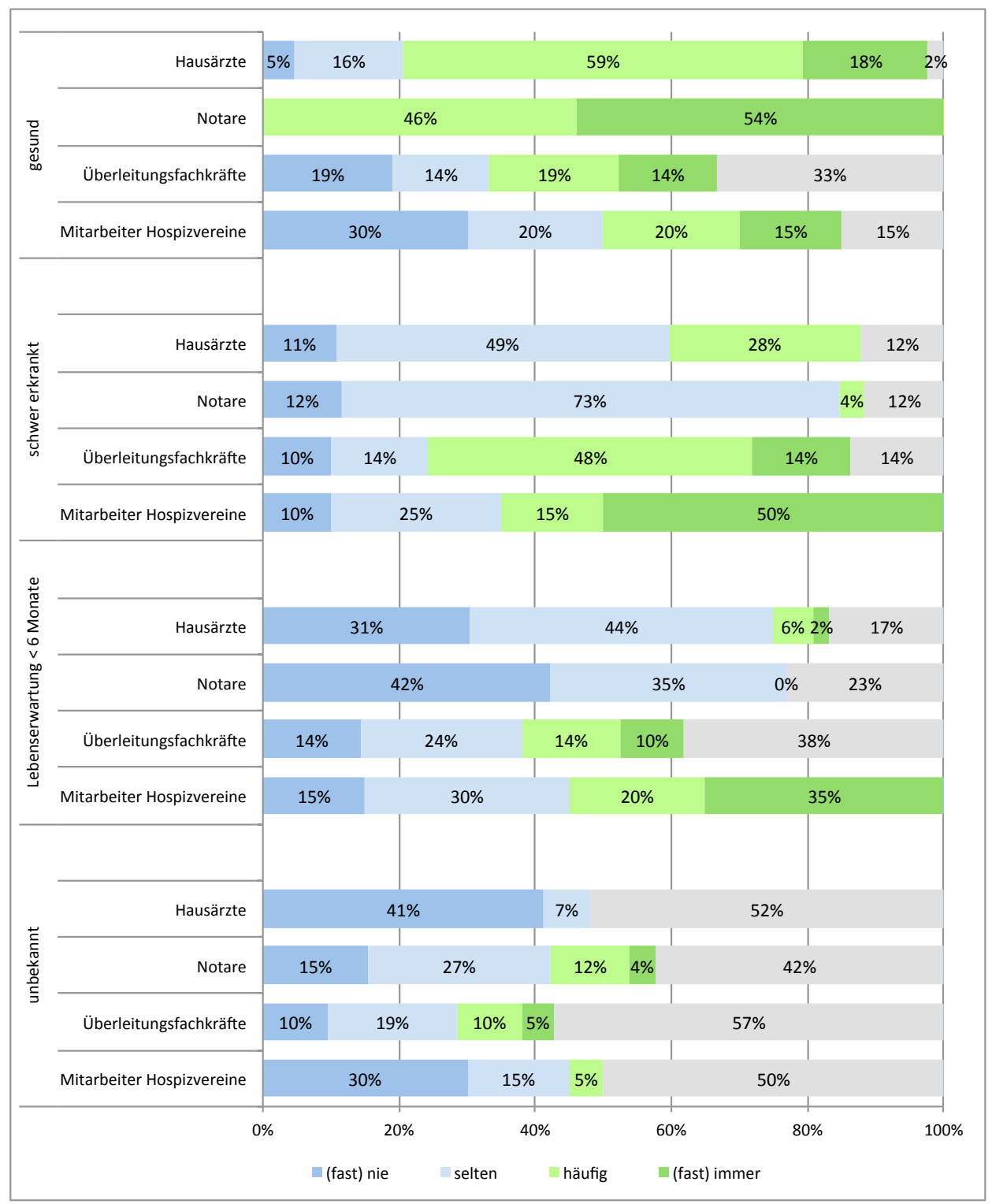


Der Schwerpunkt der Beratung durch Ärzte lag bei Ratsuchenden, die nach Einschätzung der Ärzte gesund waren (h/i=77\%). Die Beratung von bereits schwer erkrankten Menschen spielte eine deutlich geringere Rolle: Weniger als ein Drittel der befragten Ärzte erklärte, häufig schwerkranke Menschen zu beraten; weniger als $10 \%$ gaben an, häufig oder fast immer Menschen mit begrenzter Lebenserwartung zu beraten. $60 \%$ der Ärzte äußerten, der Gesundheitszustand der Ratsuchenden sei selten oder fast nie „schwer erkrankt“. Drei Viertel der Ärzte kreuzten an, eine „Lebenserwartung unter 6 Monaten“ sei bei den Ratsuchenden selten oder fast nie gegeben.

Aus den Angaben der Notare wurde klar ersichtlich, dass durch sie fast ausschließlich Menschen, die nach Kenntnis der Notare gesund sind, beraten wurden (h/i=100\%). Ein Großteil (85\%) der Notare gab an, die Ratsuchenden seien fast nie und nur selten schwer erkrankt. Eine Beratung von Menschen mit einer Lebenserwartung von weniger als sechs Monaten erfolgte bei keinem der Notare häufig oder fast immer, sondern nur selten (35\%) oder fast nie (42\%). Eine große Gruppe (42\%) führte aus, diesen Personenkreis fast nie zu beraten.

Bemerkenswert war, dass einige dieser Berater angaben, häufig (12\%) oder fast immer (4\%) keine Kenntnis über den Gesundheitszustand der Ratsuchenden zu haben.

Deutlich anders stellt sich die Situation bei der Beratung durch Überleitungsfachkräfte dar: Hier ist die Gruppe der schwer erkrankten Menschen die größte Gruppe der Beratenen (h/i 62\%). Erkennbar kleiner war bereits die Gruppe, deren Gesundheitszustand von den Beratern als gesund benannt wurde (h/i 33\%). Die Beratung von Menschen mit einer Lebenserwartung unter sechs Monaten gaben nur 14\% der Berater als häufig und $10 \%$ mit fast immer an (h/i 24\%). 15\% ist der Gesundheitszustand der Ratsuchenden häufig oder fast immer unbekannt.

Ähnlich schilderten die Mitarbeiter der Hospizvereine die Situation. Die Hälfte der Mitarbeiter von Hospizvereinen gab den Gesundheitszustand 
der bei innen Ratsuchenden mit fast immer schwer erkrankt an (50\%), weitere $15 \%$ mit häufig schwer erkrankt an. Die zweitgrößte Gruppe der dort Ratsuchenden bildeten Menschen mit begrenzter Lebenserwartung (h/i 55\%). Weniger als ein Drittel der Befragten erklärte, der Gesundheitszustand der zu Beratenden sei häufig oder fast immer gesund (h/i 35\%).

\subsubsection{Alter der Ratsuchenden}

Mit Ausnahme der Überleitungsfachkräfte gaben alle Beratergruppen die 61 bis 80 -jährigen als mit Abstand größte beratene Altersgruppe an (Abb.5).

Abbildung 5: Alter der meisten Ratsuchenden

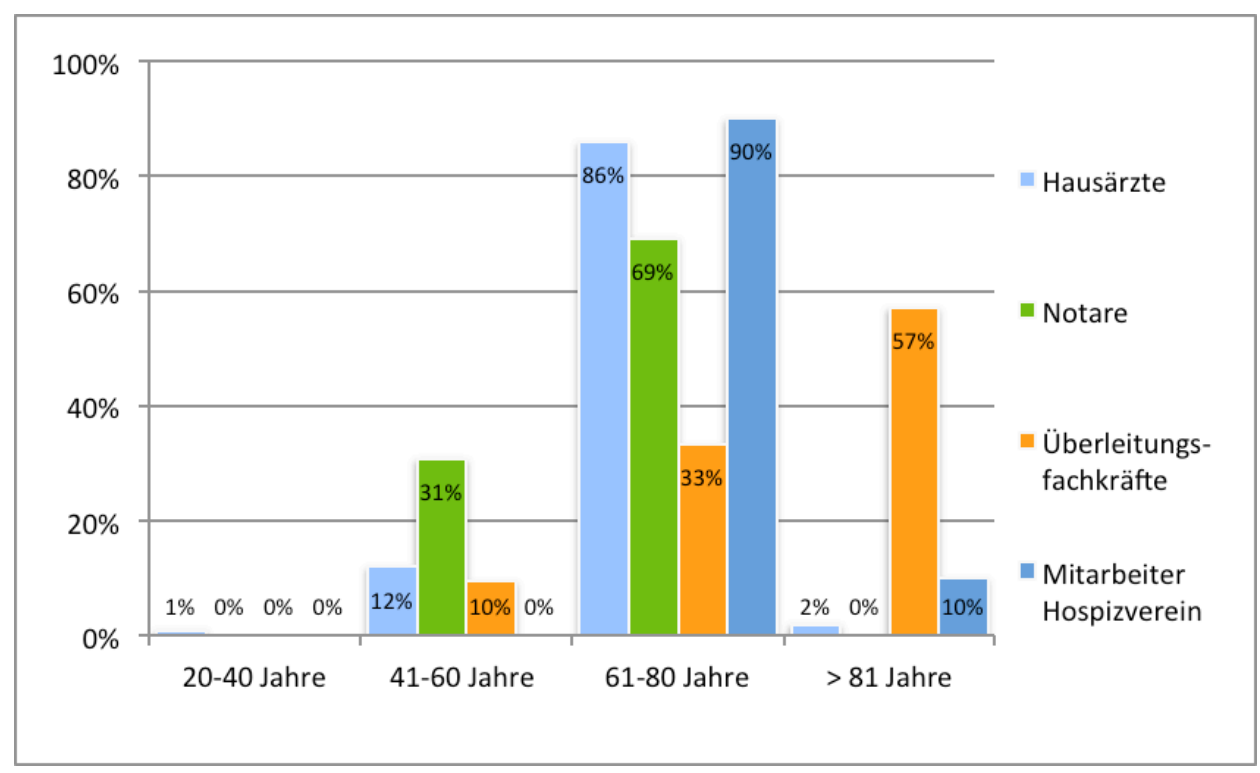

Die über 81-jährigen wurden bei über der Hälfte der Überleitungsfachkräfte und von $10 \%$ der Hospizmitarbeiter als größte Gruppe der Ratsuchenden benannt.

Knapp ein Drittel der Notare führte die 41 bis 60-jährigen als größte Gruppe der Ratsuchenden an. 


\subsubsection{Anzahl der Gespräche}

Über die Hälfte aller Berater kreuzte an, meistens zwei Gespräche pro Beratung zu führen (Abb.6). Bei ca. einem Drittel der Befragten umfasste die Beratung meistens ein Gespräch.

Abbildung 6: Alle Teilnehmer ( $n=198)$ : Anzahl der Gespräche die eine Beratung meistens umfasst

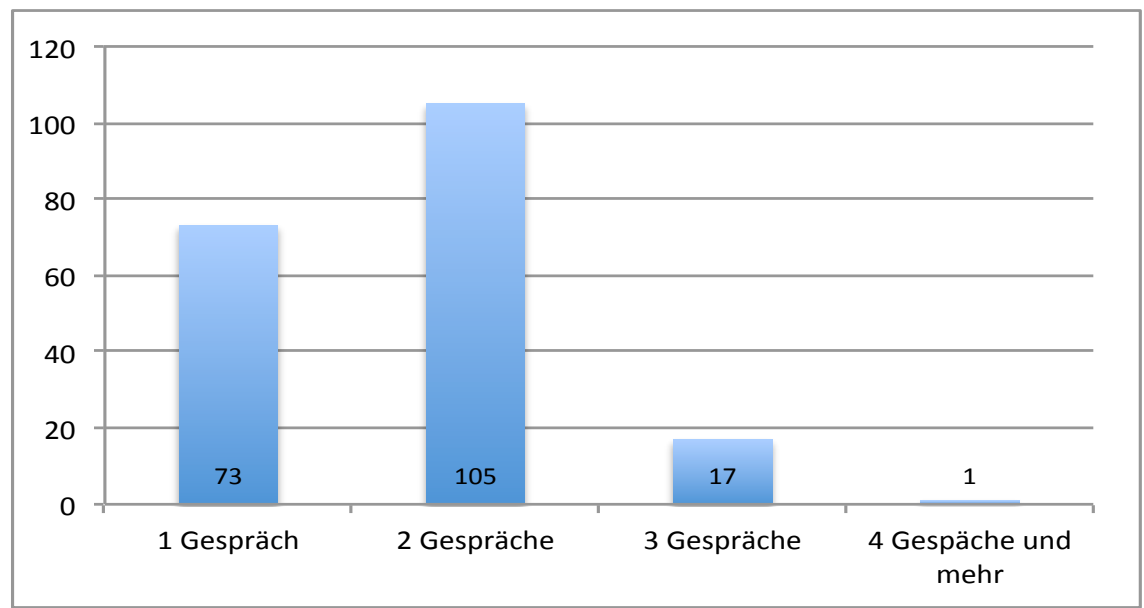

Differenzierte man bei der Betrachtung weiter nach den verschiedenen Beratergruppen, so ergab sich ein heterogenes Bild (Abb.7): 
Abbildung 7: Anzahl der Gespräche innerhalb einer Beratung

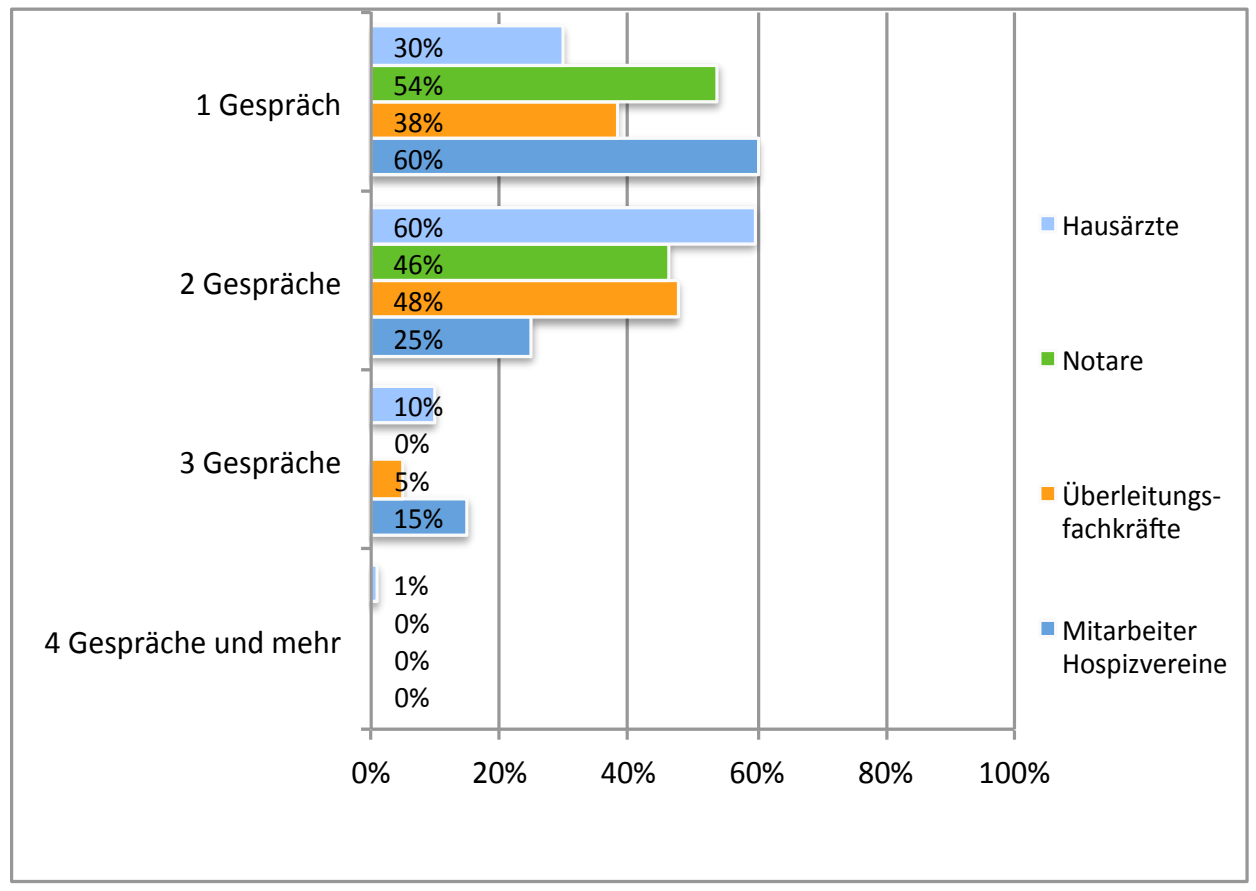

Die Anzahl der geführten Gespräche pro Beratung lag bei allen Gruppen weit überwiegend bei 1 bis 2 Gesprächen, wobei Hausärzte und Überleitungsfachkräfte eine Tendenz zu 2 und mehr Gesprächen erkennen lassen. Bei einigen Mitarbeitern von Hospizvereinen (15\%) und Ärzten (10\%) kam es zu 3 Gesprächen. 


\subsubsection{Dauer der Beratungen}

Ein weiteres, wesentliches Merkmal der Beratungsstruktur ist die dafür benötigte Zeit (Abb.8).

Abbildung 8: Dauer der Beratung insgesamt

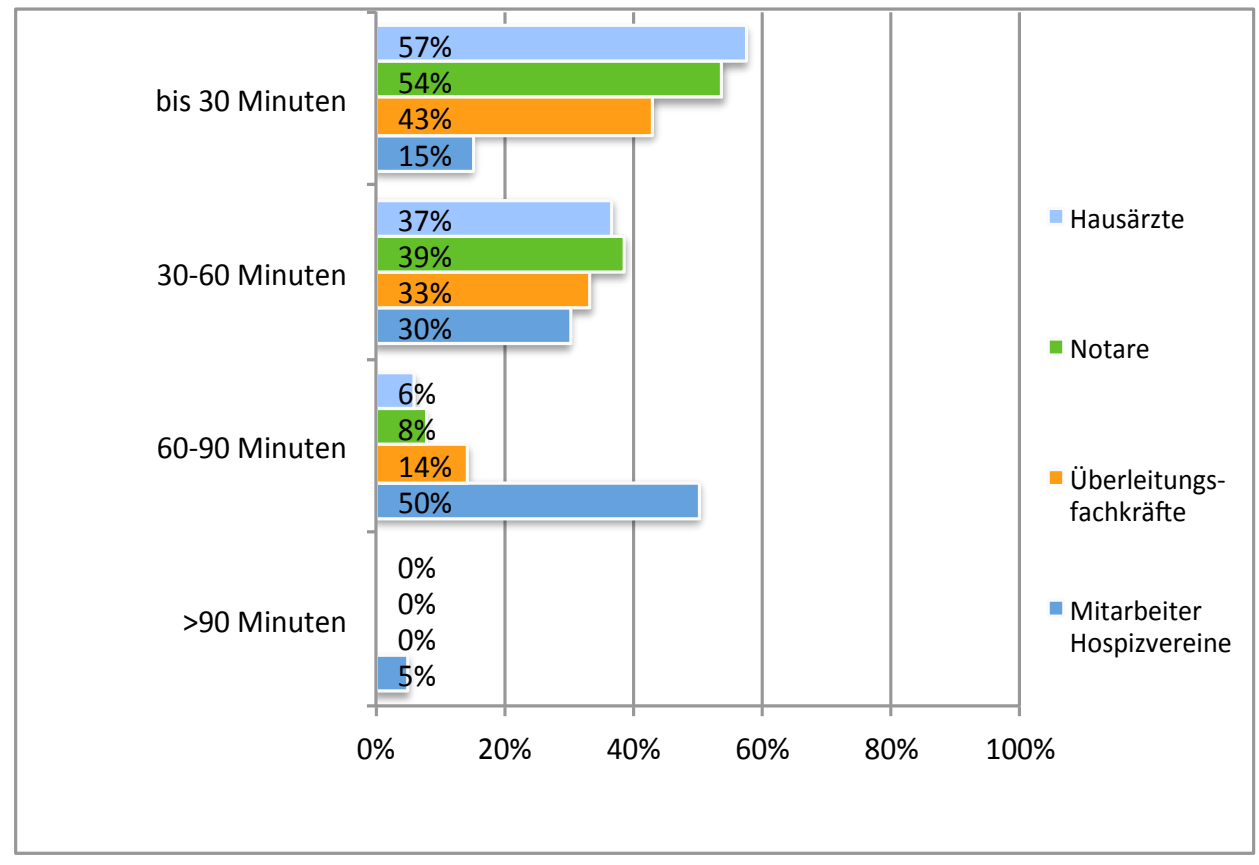

Für eine Beratung benötigten alle Gruppen, mit Ausnahme der Hospizmitarbeiter, insgesamt überwiegend bis zu 30 Minuten (43-57\%), vielfach aber auch 30 bis 60 Minuten (33-39\%). Bei den Hospizmitarbeitern verwendete die Hälfte der Berater 60 bis 90 Minuten für eine Beratung. Die Dauer der Gespräche war innerhalb der Gruppen sehr heterogen. 


\subsubsection{Verwendete Formulare}

Die Frage nach den in der Beratung verwendeten Formularen gab folgenden Einblick in den Inhalt der Beratung (Abb.9):

Abbildung 9: Alle Teilnehmer $(n=198)$ : Häufigkeit der Nennung der verschiedenen Formulare/Texte

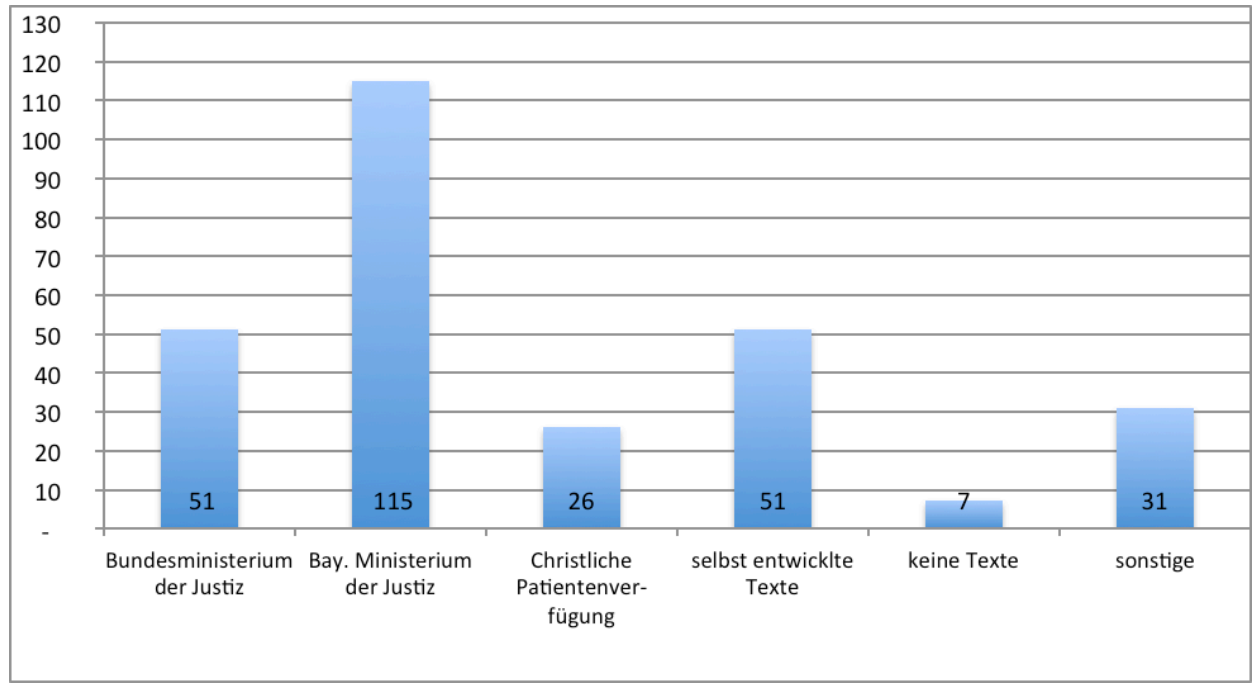

Mehrfachnennungen möglich

Das Formular des Bayerischen Staatsministeriums der Justiz war das am häufigsten benannte Formular. Es wurde von über der Hälfte der befragten Personen verwendet. Die Textbausteine des Bundesministeriums der Justiz setzte ca. jeder fünfte der befragten Berater ein. Jeder fünfte Berater gestaltete eigene Formulare/Texte. 
Betrachtet man die Nutzung der Formulare nach Beratergruppen lassen sich folgende Aufteilungen erkennen (Abb. 10).

Abbildung 10: Formulare/Texte anhand derer die Berater beraten

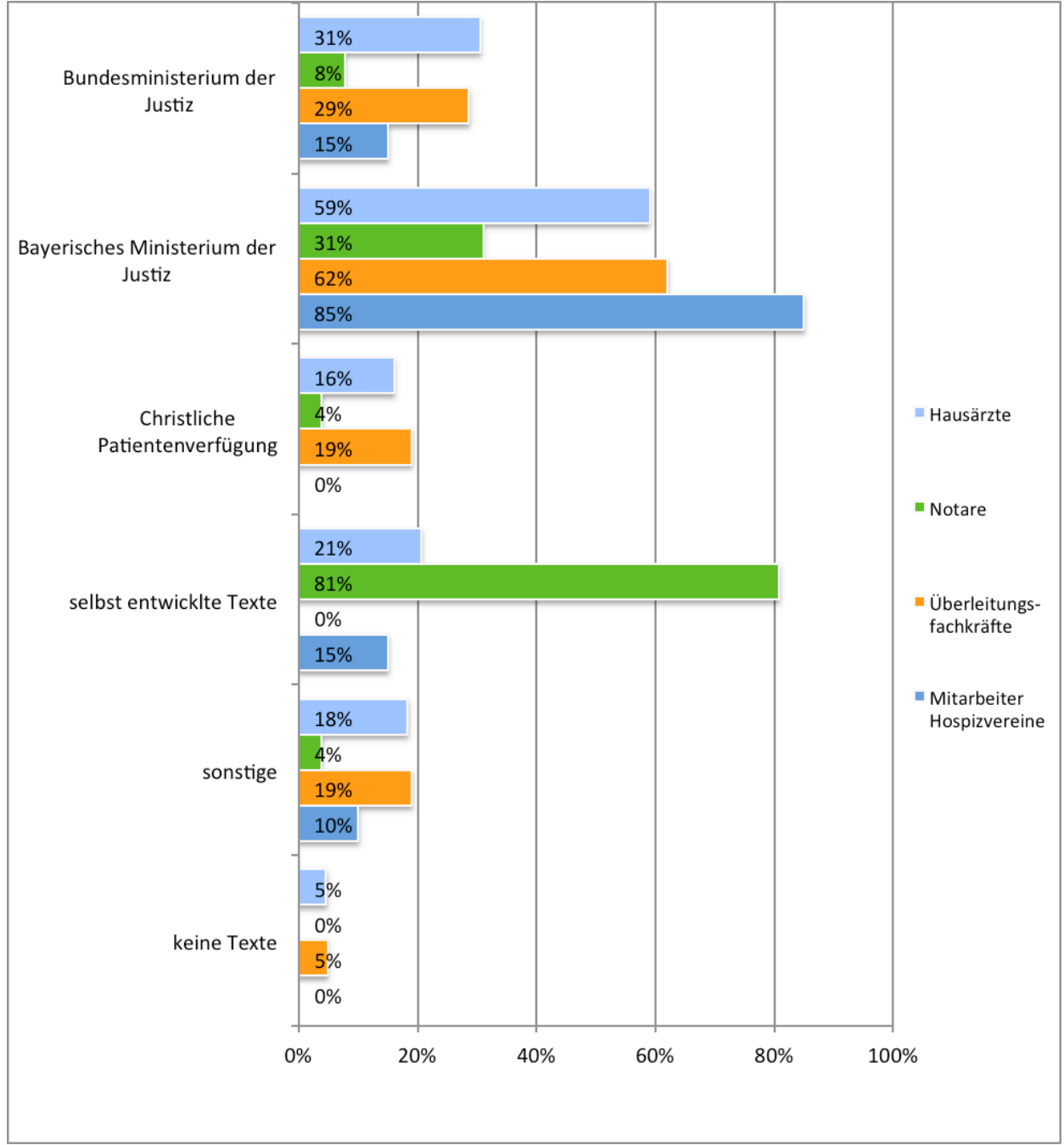

Mehrfachnennungen möglich

Mit Ausnahme der Notare kreuzten alle Gruppen am häufigsten die Verwendung des Formulars des Bayerischen Staatsministeriums der Justiz an. Bei den Notaren wurde dieses Formular, nach den selbst erstellten Formularen, am zweithäufigsten aufgeführt.

Bei den Ärzten folgten nach dem Formular des Bayerischen Ministeriums der Justiz (59\%) an zweiter Stelle die Textbausteine des Bundesjustizministeriums (31\%). Relativ hoch war der Anteil der Ärzte, die daneben die 
Verwendung selbst entwickelter Formulare angaben (21\%). Auch die Christliche Patientenverfügung (16\%), sowie sonstige Texte (18\%) spielten in ihrer Beratung eine Rolle. Bei den "sonstigen Texten“ wurden verschiedenste Angaben gemacht (s.u.). Eine kleine Gruppe von Ärzten (5\%) beriet ohne die Verwendung von Formularen/Texten.

Notare arbeiteten weit überwiegend anhand selbst entwickelter Texte (81\%), bemerkenswerter Weise aber zu knapp einem Drittel auch anhand des Formulars des Bayerischen Staatsministeriums der Justiz. Alle anderen Formulare/Texte wurden kaum genannt.

Auch bei den Überleitungsfachkräften standen die Textbausteine des Bundesjustizministeriums (29\%) an zweiter Stelle, gefolgt von der Christlichen Patientenverfügung (19\%) und sonstigen Texten. Selbst entwickelte Texte wurden nicht verwendet. Ein Berater dieser Gruppe (5\%) beriet auch ohne die Verwendung von Texten.

Die Mitarbeiter der Hospizvereine benannten das Formular des Bayerischen Staatsministeriums der Justiz von allen befragten Gruppen am weitaus häufigsten (85\%). Wesentlich seltener wurde eine Beratung anhand der Textbausteine des Bundesjustizministeriums (15\%) oder sonstiger Texte (10\%) angeben. Die Christliche Patientenverfügung spielte bei dieser Beratergruppe überhaupt keine Rolle. Erkennbar wurde, dass 15\% dieser Berater selbst entwickelte Texte verwendeten.

Bei den Angaben zu den „sonstigen Formlaren/Texten“ (Tab.7) ist zu vermuten, dass hier in einigen Fällen (Nr. 2, 16, 17) die Verwendung des Formulars des Bayerischen Staatsministeriums der Justiz gemeint war. Damit würde sich die Häufigkeit der Verwendung dieses Formulars weiter erhöhen. 


\begin{tabular}{|c|l|l|l|}
\hline 1 & $\begin{array}{l}\text { Mitarbeiter } \\
\text { Hospizverein }\end{array}$ & Landeshauptstadt München & 1 \\
\hline 2 & & PV für den Fall schwerer Erkrankung & 1 \\
\hline 3 & $\begin{array}{l}\text { Überleitungs- } \\
\text { fachkräfte }\end{array}$ & Landeshauptstadt München & 1 \\
\hline 4 & & Münchner Betreuungsstelle & 1 \\
\hline 5 & & $\begin{array}{l}\text { Senatsverwaltung für Justiz und Verbrau- } \\
\text { cherschutz }\end{array}$ & 1 \\
\hline 6 & & unterschiedliche & 1 \\
\hline 7 & Notare & Muster Prof. Borasio & 1 \\
\hline 8 & Ärzte & Vordrucke von Pharmafirmen & 3 \\
\hline 9 & & vom Patienten mitgebrachte Vordrucke & 3 \\
\hline 10 & & Internetformulare allgemein & 3 \\
\hline 11 & & Patientenverfügung.de & 2 \\
\hline 12 & & Landeshauptstadt München & 2 \\
\hline 13 & & Malteser & 2 \\
\hline 14 & & Text der Bundesärztekammer & 1 \\
\hline 15 & & Notarversionen & 1 \\
\hline 16 & & Bayerisches Ministerium für Familien & 1 \\
\hline 17 & & Beck-Verlag & 1 \\
\hline 18 & & Kohlhammer Verlag & 1 \\
\hline 19 & & diverse Vordrucke & 1 \\
\hline 20 & & Firmenvorlagen & 1 \\
\hline 21 & & gesammelte Unterlagen & 1 \\
\hline 22 & & MHD & 1 \\
\hline
\end{tabular}

Tabelle 7: Sonstige verwendete Formulare/Texte

3.2.7 Verwendung von durch den Ratsuchenden mitgebrachten Formularen

Mit den verwendeten Vordrucken befasste sich die Frage, ob auch anhand von durch den Ratsuchenden mitgebrachten Formularen, die der Berater sonst nicht verwendet, beraten wird.

Durch sie sollte ein Einblick gewonnen werden, inwieweit sich die Berater auf die Wünsche des Ratsuchenden nach der Verwendung von bestimmten Vordrucken einließen. 
Abbildung 11: Beratung anhand von durch den Ratsuchenden mitgebrachten, i.d.R. vom Berater nicht verwendeten Formularen

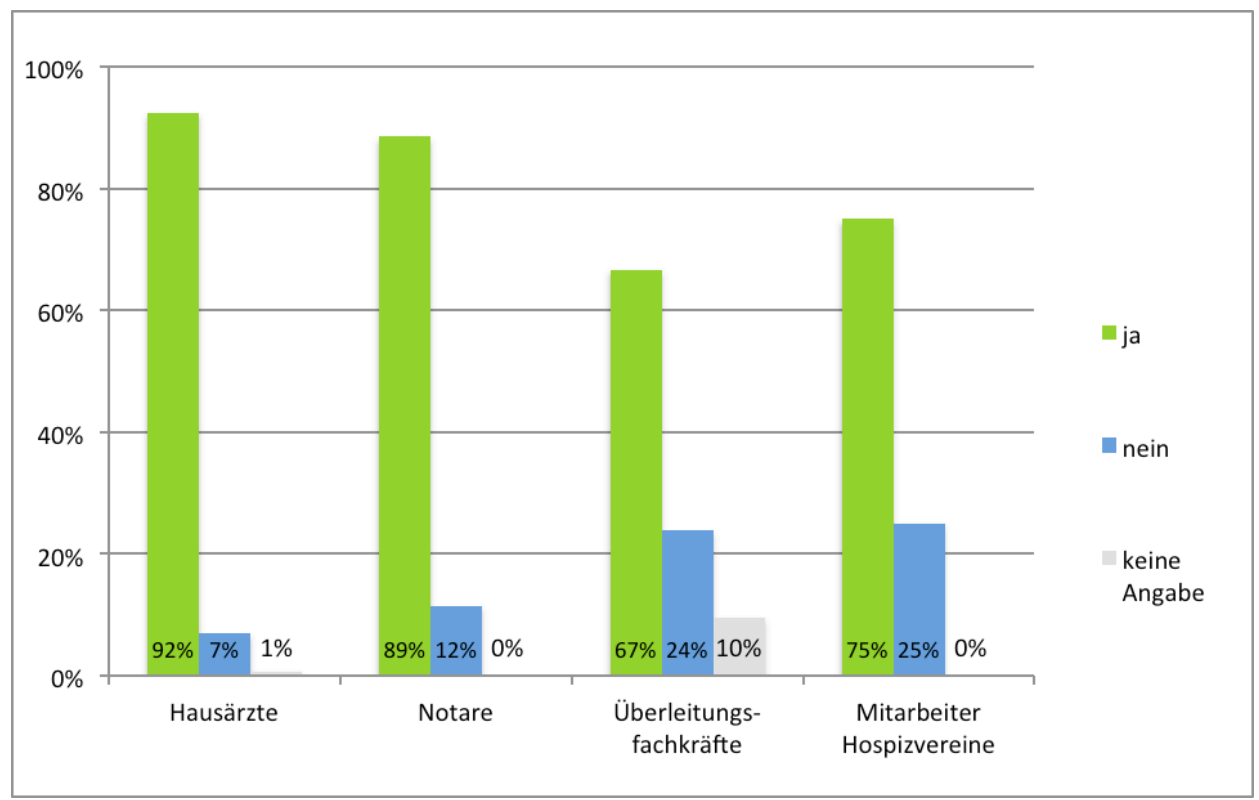

Die ermittelten Ergebnisse zeigten deutlich, dass der Großteil (67-91\%) der Berater auf Wunsch des Ratsuchenden auch anhand "fremder" Formulare beriet.

Die größte Zurückhaltung war bei den Überleitungsfachkräften (24\%) und den Mitarbeitern der Hospizvereine (25\%) zu erkennen, bei denen jeweils ca. ein Viertel der Berater ankreuzte, nicht anhand fremder Formulare zu beraten. 


\subsubsection{Fertigstellung der Patientenverfügung}

Die Hausärzte bildeten die Gruppe, die am häufigsten von der Fertigstellung der Patientenverfügung innerhalb der Beratung berichtete (51\%).

Abbildung 12: Häufigkeit der Fertigstellung der Patientenverfügung innerhalb der Beratung

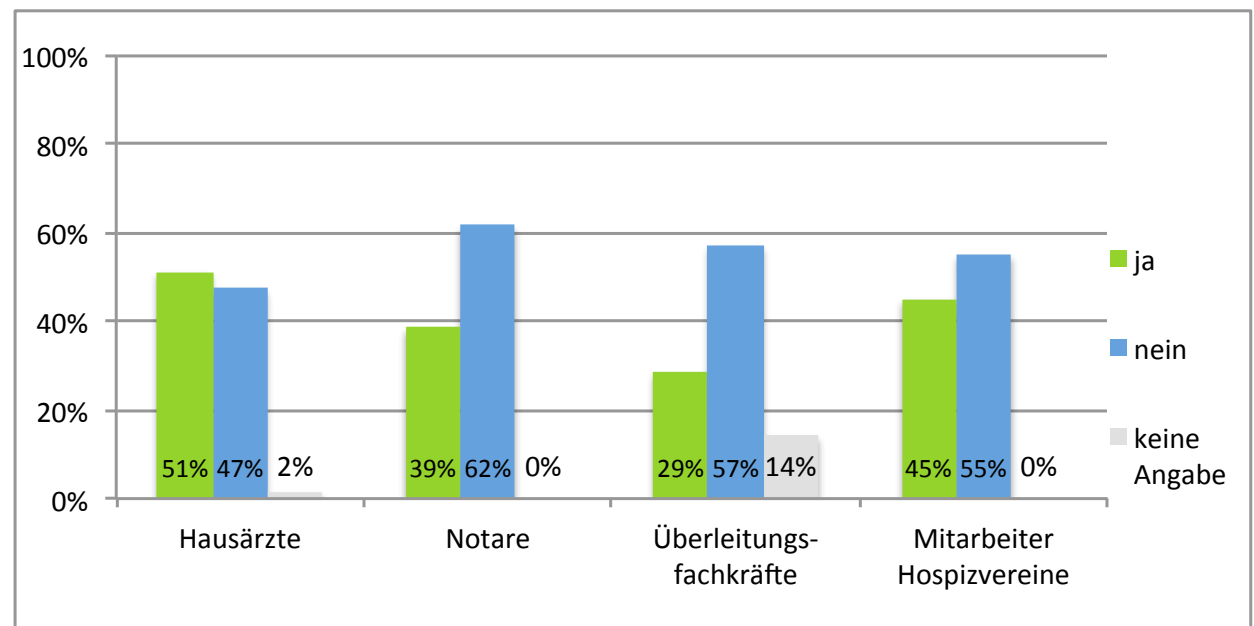

Gefolgt wurde dieser Gruppe durch die Mitarbeiter der Hospizvereine $(45 \%)$ und die Notare (39\%). Am geringsten war die Quote der fertigen Patientenverfügungen bei den Überleitungsfachkräften (29\%).

Bei mehr als der Hälfte der Mitarbeiter von Hospizvereinen, Überleitungsfachkräften und sogar $62 \%$ der Notare wurde deutlich, dass die Patientenverfügung nicht innerhalb der Beratung fertig erstellt wurde. Bei den Ärzten war der Prozentsatz der nicht fertig verfassten Verfügungen mit $47 \%$ etwas geringer.

Diese Daten zeigten, dass die Häufigkeit der Fertigstellung der Patientenverfügungen sowohl zwischen den Gruppen, als auch innerhalb der Gruppen deutlich divergierte.

Ein Zusammenhang zwischen der Anzahl der geführten Gespräche und der Häufigkeit der fertiggestellten Patientenverfügungen ließ sich auch bei einer vertieften Auswertung der Daten nicht belegen (Tab.8). 


\begin{tabular}{|l|l|c|c|c|}
\hline \multirow{4}{*}{$\begin{array}{l}\text { Wieviele Gespräche umfasst eine } \\
\text { Beratung meistens? }\end{array}$} & \multicolumn{4}{l}{\begin{tabular}{l}
\multicolumn{3}{l}{ Wird die Patientenverfügung in der Regel mit dem } \\
Ratsuchenden innerhalb der Beratung fertig erstellt?
\end{tabular}} \\
\cline { 2 - 5 } & & ja & nein & keine Angabe \\
\cline { 2 - 5 } & 1 Gespräch & 31 & 42 & 0 \\
\cline { 2 - 5 } & 2 Gespräche & 50 & 53 & 0 \\
\cline { 2 - 5 } & 3 Gespräche & 10 & 6 & 2 \\
\cline { 2 - 5 } & 4 Gespräche & 1 & 0 & 0 \\
\cline { 2 - 5 } & keine Angabe & 0 & 0 & 5 \\
\hline
\end{tabular}

Tabelle 8: Zusammenhang Anzahl Gespräche - Fertigstellung der Patientenverfügung

\subsubsection{Hinweise für die Aufbewahrung der Patientenverfügung}

Um den Einfluss der Berater auf die Auffindbarkeit der Patientenverfügungen ermitteln zu können wurde gefragt, ob Empfehlungen für die Aufbewahrung des Dokumentes gegeben werden (Abb.13).

Abbildung 13: Häufigkeit von Tipps zur Aufbewahrung der Patientenverfügung

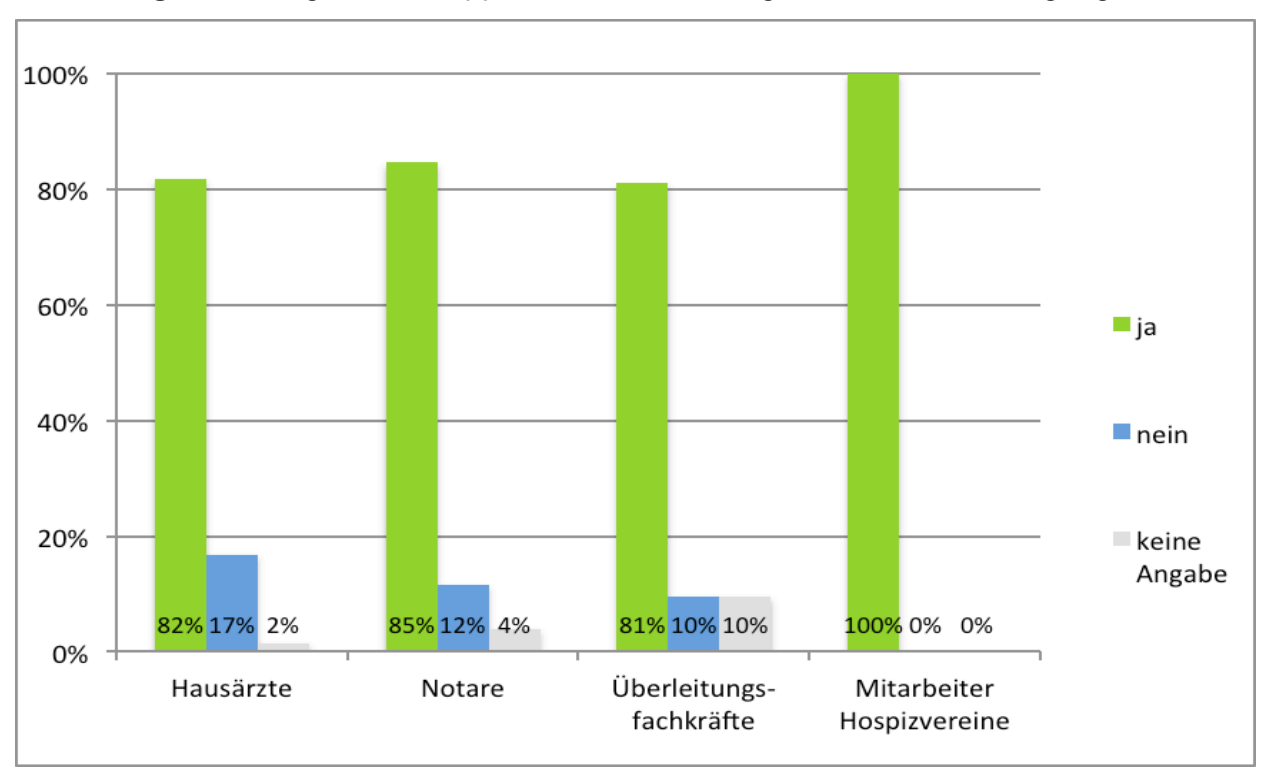

Über 80\% aller Berater gaben Hinweise zur Aufbewahrung der Patientenverfügung. Die Hausärzte stellten mit $17 \%$, vor den Notaren $(12 \%)$ und den Überleitungsfachkräften (10\%) die größte Gruppe derjenigen, die keine Hinweise zur Aufbewahrung kommunizierten. 
Der inhaltliche Schwerpunkt aller in einem offenen Feld angegeben Hinweise lag auf der Information von Angehörigen/ Freunden/ Vertrauenspersonen (Tab.9).

\begin{tabular}{|l|c|c|c|c|}
\hline & $\begin{array}{c}\text { Hausärzte } \\
(\mathrm{n}=131)\end{array}$ & $\begin{array}{c}\text { Notare } \\
(\mathrm{n}=26)\end{array}$ & $\begin{array}{c}\text { Uberleitungs- } \\
\text { fachkräfte } \\
(\mathrm{n}=21)\end{array}$ & $\begin{array}{c}\text { Mitarbeiter } \\
\text { Hospizverein } \\
(\mathrm{n}=20)\end{array}$ \\
\hline Aufbewahrung Zuhause & 37 & 1 & 2 & 4 \\
\hline Kopie beim Arzt & 62 & 3 & 6 & 6 \\
\hline $\begin{array}{l}\text { Info/Kopie/Original an } \\
\text { Angehörige/Freunde/ } \\
\text { Vertrauenspersonen }\end{array}$ & 38 & - & 1 & 1 \\
\hline Info/Kopie/Original an Vertreter & 15 & 7 & 7 & 10 \\
\hline Kopie an Institution (Heim) & - & - & 8 & 2 \\
\hline beim Notar & 14 & 1 & 1 & - \\
\hline Zentrales Vorsorgeregister & 3 & 10 & - & - \\
\hline Zugriff gewährleistet & 15 & 7 & - & 1 \\
\hline Karte im Geldbeutel & 7 & 3 & - & 3 \\
\hline
\end{tabular}

Tabelle 9: Tipps zur Aufbewahrung der Patientenverfügung im Einzelnen

Die Ärzte erwähnten eine Kopie an sich selbst (62 Nennungen) häufiger als die Information von Angehörigen/Vertrauenspersonen (38 Nennungen) oder Vertretern (15 Nennungen). Die Notare benannten primär die Registrierung im Zentralen Vorsorgeregister der Bundesnotarkammer (ZVR), die Überleitungsfachkräfte die Kopie an das Heim. Die Mitarbeiter von Hospizvereinen wiesen hauptsächlich auf die Bedeutung der Information des Vertreters hin. 


\subsubsection{Inhalt der Beratungsgespräche im Einzelnen}

Der Inhalt der Beratungsgespräche stellte sich als sehr heterogen dar (Abb.14).

Abbildung 14: Inhalte der Beratung allgemein

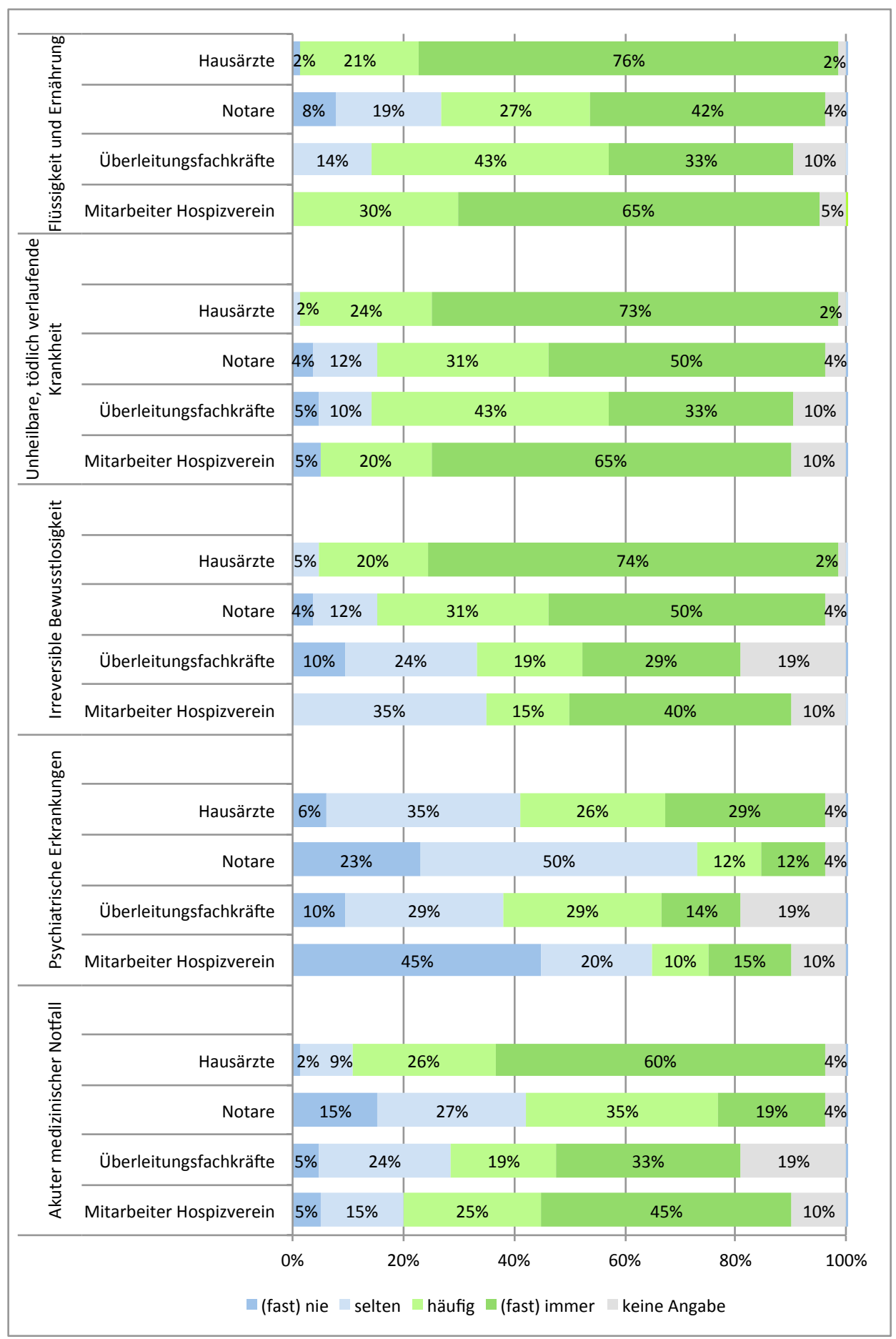


Am höchsten war die Beratungshäufigkeit aller Beratergruppen bei den Themen „Unheilbare, tödlich verlaufende Krankheit“ (h/i 76-97\%), „Flüssigkeit und Ernährung“ (h/i 69-97\%), gefolgt von „Irreversible Bewusstlosigkeit“ (h/i 48-94\%). Deutlich weniger Eingang in die Beratung fanden Erläuterungen zu „Akuter medizinischer Notfall“ (h/i 52-86\%) und vor allem „Psychiatrische Erkrankungen“ (h/i 24-55\%).

Bei den Ärzten zeichnet sich ein klares, vergleichsweise homogenes Bild der Beratung ab. Der Schwerpunkt lag bei den Themen „Flüssigkeit und Ernährung“ und „Unheilbare Krankheit“ (h/i 97\%), dicht gefolgt von den Themen „Irreversible Bewusstlosigkeit“ (h/i 94\%) und „Akuter medizinischer Notfall“ (h/i 86\%). Eine Beratung zum Thema „Psychiatrische Erkrankungen" nahm ca. die Hälfte der Ärzte häufig oder immer vor (55\%).

Die notarielle Beratung erfasste das Thema "irreversible Bewusstlosigkeit“ in gleicher Häufigkeit, wie „Unheilbare Krankheit“ (h/i 81\%). Erst nachfolgend häufig wurden die Themen „Flüssigkeit und Nahrung“ (h/i 69\%), „Medizinischer Notfall“ (h/i 54\%) einbezogen. Das Thema „Psychiatrische Erkrankung" fand nach Aussage von knapp drei Vierteln der Notare (n/s 73\%) fast nie oder selten Eingang in die Beratung, bei dem verbleibenden Viertel jedoch häufig oder fast immer ( $h / s$ 24\%).

Von den Überleitungsfachkräften wurden die Themen „Flüssigkeit und Ernährung“, sowie „Unheilbare, tödlich verlaufende Krankheit“ (h/i 76\%) als weitaus häufigste Beratungsgegenstände benannt. Die Beratung zum Thema „Akuter medizinscher Notfall“ (h/i 52\%) stand daran anschließend an zweiter Stelle, das der „Irreversiblen Bewusstlosigkeit“ an dritter (h/i $48 \%$ ). Fragen um „Psychiatrische Erkrankungen“ bezog knapp die Hälfte dieser Berater häufig oder fast immer ein (h/i 43\%).

Sichtbar wurde, dass es innerhalb dieser Gruppe zwar Beratungsschwerpunkte gab, aber zugleich relativ große Teile dieser Gruppe zu den gleichen Themen auch nur selten oder fast nie berieten. Besonders deutlich kam dies im Bereich der „Psychiatrischen Erkrankungen“ zum Ausdruck: $43 \%$ der Berater kreuzten an, häufig oder fast immer dazu zu beraten, 
$39 \%$ der Berater aber selten oder fast nie. Ähnlich, wenn auch nicht ganz so klar, zeichnete sich diese Tendenz bei den Themen „Irreversible Bewusstlosigkeit“ und „Akuter medizinische Notfall“ ab.

Die Beratungsschwerpunkte bei den zur Auswahl gestellten Themen sahen die Mitarbeiter der Hospizvereine ebenfalls eindeutig auf den Themen „Flüssigkeit und Ernährung am Lebensende“, sowie „Unheilbare, tödlich verlaufende Krankheit“ ( $\mathrm{h} / \mathrm{i}>85 \%$ ) liegen. Der „Akute medizinische Notfall“ erhielt bei über der Hälfte dieser Berater fast immer Eingang in die Beratung (h/i 70\%). Beim Thema „Irreversible Bewusstlosigkeit“ war die Beratungshäufigkeit innerhalb der Gruppe am unterschiedlichsten: 40\% der Berater erklärten, diesen Bereich fast immer einzubeziehen, 35\% thematisierten inn selten. Das Feld der „Psychiatrischen Erkrankungen“ wurde vom Großteil dieser Berater fast nie oder selten beraten (n/s $65 \%$ ), ein Viertel sprach jedoch häufig oder fast immer darüber. 


\subsubsection{Information zu anderen Vorsorgedokumenten}

Über die Themen „Vorsorgevollmacht“ (h/i 90-100\%) und, etwas nachgelagert, „Betreuungsverfügung“ (h/i 70-89\%) wurde von allen Gruppen überwiegend informiert (h/i 90-100\%) (Abb.15).

Abbildung 15: Häufigkeit von Information zu anderen Vorsorgedokumenten

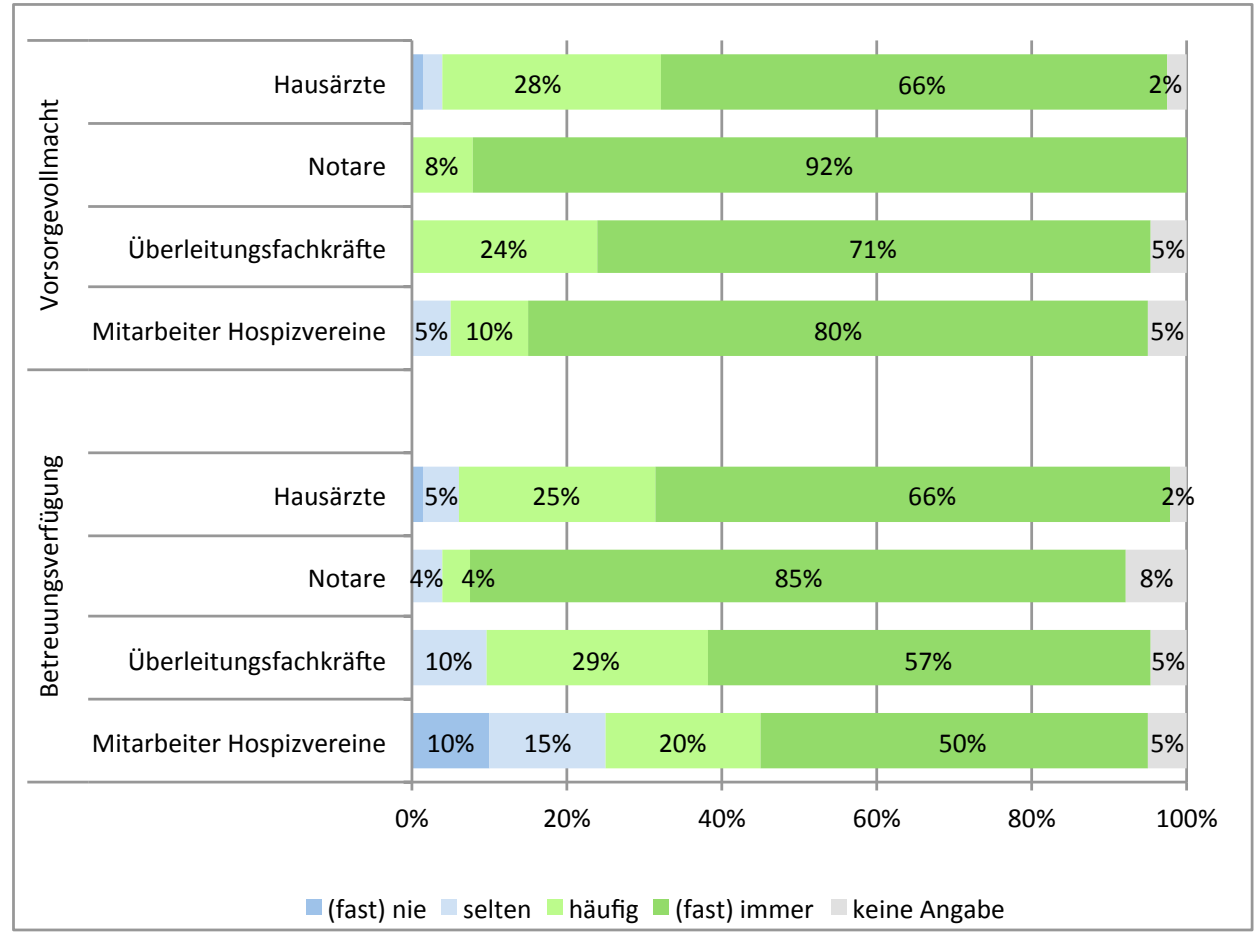

Bei den Ärzten spielte die Information zu Vorsorgevollmacht und Betreuungsverfügung eine große Rolle in der Beratung ( $\mathrm{h} / \mathrm{i}$ 94\%). Das Thema Vorsorgevollmacht scheint jedoch kein in gleicher Weise fester Bestandteil, wie bei den anderen Gruppen zu sein, da der Anteil der Ärzte, die fast immer zur Vorsorgevollmacht informierten (66\%) geringer war als in den anderen Gruppen (71-92\%).

Über Vorsorgevollmachten informierten fast alle Notare und auch fast immer (i 92\%). Ebenso war die Information zur Betreuungsverfügung fester Bestandteil der Beratung (i 85\%).

Der Anteil der Überleitungsfachkräfte, die fast immer oder häufig über eine Vorsorgevollmacht informierten, lag mit 95\% ebenfalls sehr hoch. Ähnlich häufig wurde die Information zur Betreuungsverfügung genannt (h/i 86\%). 
Eine hohe Bedeutung wurde auch der Betreuungsverfügung zugemessen, da über die Hälfte der Berater fast immer und 29\% häufig darüber informierten (h/i 86\%).

Die Mitarbeiter der Hospizvereine informierten fast alle zur Vorsorgevollmacht (h/i 90\%). Die Beratungshäufigkeit im Hinblick auf die Betreuungsverfügung war bereits deutlich niedriger (h/i 70\%). Insbesondere wurde ersichtlich, dass $25 \%$ hierzu nur selten oder fast nie beraten.

3.2.12 Hinweis auf Möglichkeit eines Notfallplanes und dessen tatsächliche Erstellung

Weit weniger häufig als zu den Vorsorgedokumenten Patientenverfügung, Vorsorgevollmacht und Betreuungsverfügung erfolgte die Information zur Möglichkeit eines Notfallplanes (h/i 12-65\%) (Abb.16).

Abbildung 16: Notfallplanung

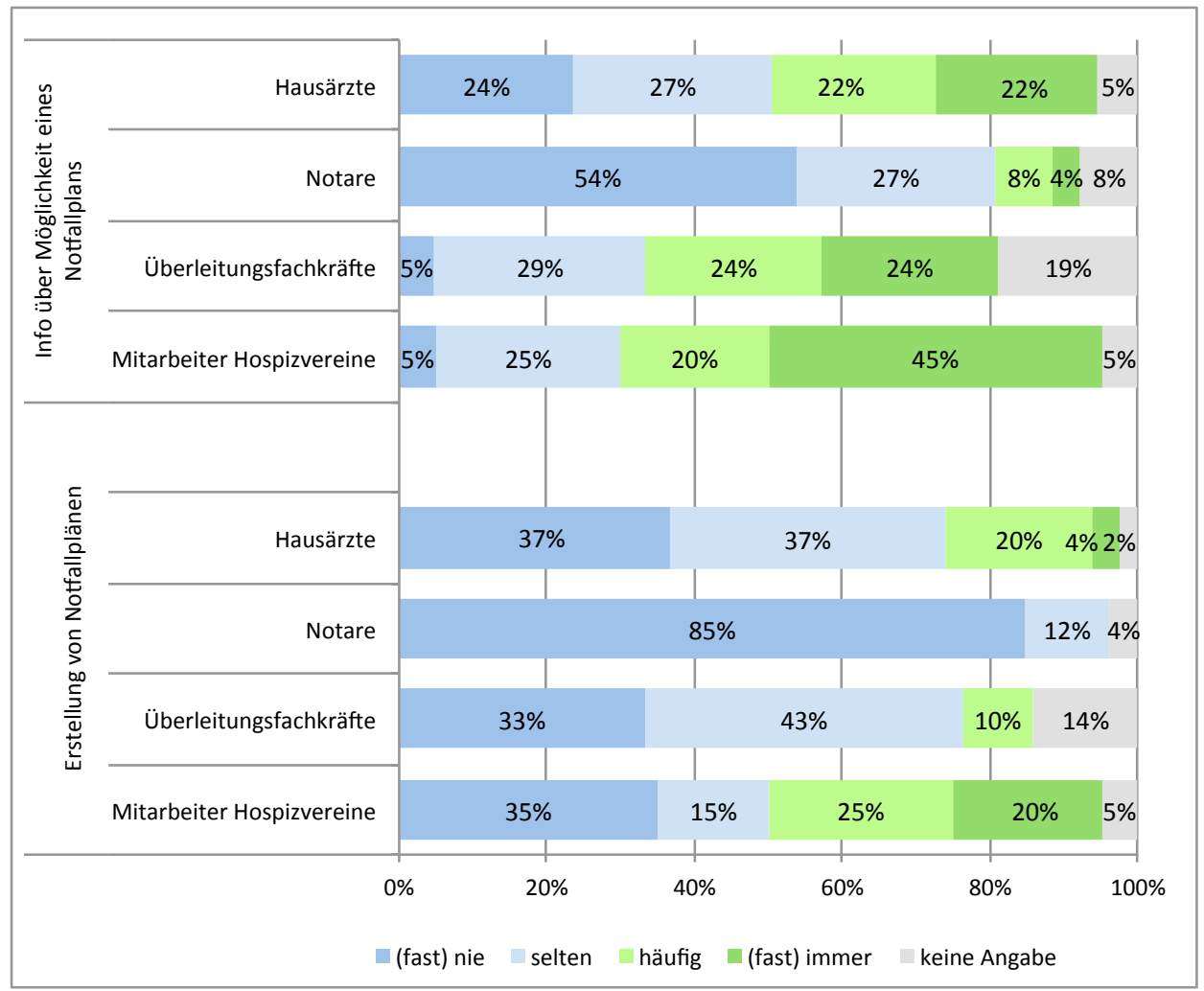


Zur Erstellung eines Notfallplanes kam es nur bei den Mitarbeitern der Hospizvereine häufiger (h/i 45\%). Bei den Beratungen der anderen Gruppen hatte die Verfassung von Notfallplänen eine weit nachgeordnete oder keine Bedeutung (h/i 0-24\%). Im Themenbereich „Notfallplan“ ist die Heterogenität der Antworten innerhalb und zwischen den Gruppen somit groß.

Bei den Ärzten ließen sich im Hinblick auf die Häufigkeit der Information zum Notfallplan ähnlich starke Gruppen erkennen (n/s 51\%, h/i 44\%). Bemerkenswert war, dass der Großteil der Ärzte selten (37\%) oder fast nie $(37 \%)$ Notfallpläne erstellte und weniger als ein Viertel häufiger an einem derartigen Prozess beteiligt war ( $\mathrm{h} / \mathrm{i} 24 \%$ ).

Selbst von den 11 Ärzten, die ankreuzten, häufig oder fast immer Menschen mit einer Lebenserwartung von weniger als 6 Monaten zu beraten, gaben nur vier an häufig Notfallpläne zu erarbeiten (Tab.10).

\begin{tabular}{|l|l|c|c|c|c|}
\hline \multirow{2}{*}{ Ärzte: } & \multicolumn{5}{|l|}{ Erarbeiten Sie auch Notfallpläne mit den Ratsuchenden? } \\
\hline \multirow{2}{*}{$\begin{array}{l}\text { Wie häufig ist der Gesundheits- } \\
\text { zustand der Ratsuchenden "Lebens- } \\
\text { erwartung absehbar begrenzt } \\
\text { (<6 Monate)"? }\end{array}$} & & (fast) nie & selten & häufig & (fast immer) \\
\cline { 2 - 7 } & (fast) nie & 24 & 10 & 5 & 1 \\
\cline { 2 - 7 } & selten & 19 & 24 & 13 & 2 \\
\cline { 2 - 7 } & häufig & 3 & 3 & 2 & 0 \\
\cline { 2 - 7 } & (fast) immer & 0 & 1 & 2 & 0 \\
\hline
\end{tabular}

Tabelle 10: Beratung von Menschen mit einer Lebenserwartung $<6$ Monate und Erstellung von Notfallplänen

Bei den Notaren spielte bereits die Information über die Möglichkeit eines Notfallplanes kaum eine Rolle: $12 \%$ der befragten Notare bezogen dieses Thema selten in die Gespräche ein. Über die Hälfte der Notare kreuzte an, fast nie zu diesem Thema zu beraten (n 54\%). Kein Notar gab an, häufig oder immer mit der Erstellung von Notfallplänen befasst zu sein.

Nur 24\% der befragten Überleitungsfachkräfte wiesen nach ihren Angaben, fast immer auf die Möglichkeit eines Notfallplanes hin. Ein weiteres knappes Viertel erteilte diesen Hinweis häufig, über ein Drittel jedoch nur selten oder fast nie. Die Beratungshäufigkeit zu diesem Thema war innerhalb der Gruppe somit recht unterschiedlich. Lediglich 10\% dieser Berater erstellten häufig Notfallpläne. 
Auf die Möglichkeit eines Notfallplanes machten nur weniger als die Hälfte der Mitarbeiter der Hospizvereine fast immer aufmerksam und ca. ein Drittel selten oder fast nie. $45 \%$ dieser Berater erklärten, häufig oder fast immer Notfallpläne zu erstellen. Die Beratungshäufigkeit divergierte bei den Themen zum Notfall innerhalb der Gruppe somit deutlich.

\subsubsection{Einbeziehung des zukünftigen Vertreters}

Über die Hälfte der Teilnehmer aller befragten Gruppen wiesen häufig oder fast immer auf die Möglichkeit der Teilnahme des zukünftigen Vertreters an der Beratung hin (Abb.17). Die Häufigkeit des Hinweises divergierte zwischen den verschiedenen Gruppen jedoch deutlich (h/i 53-86\%).

Abbildung 17: Hinweis auf die Teilnahmemöglichkeit zukünftiger Vertreter und tatsächliche Teilnahme dieser Personen an der Beratung

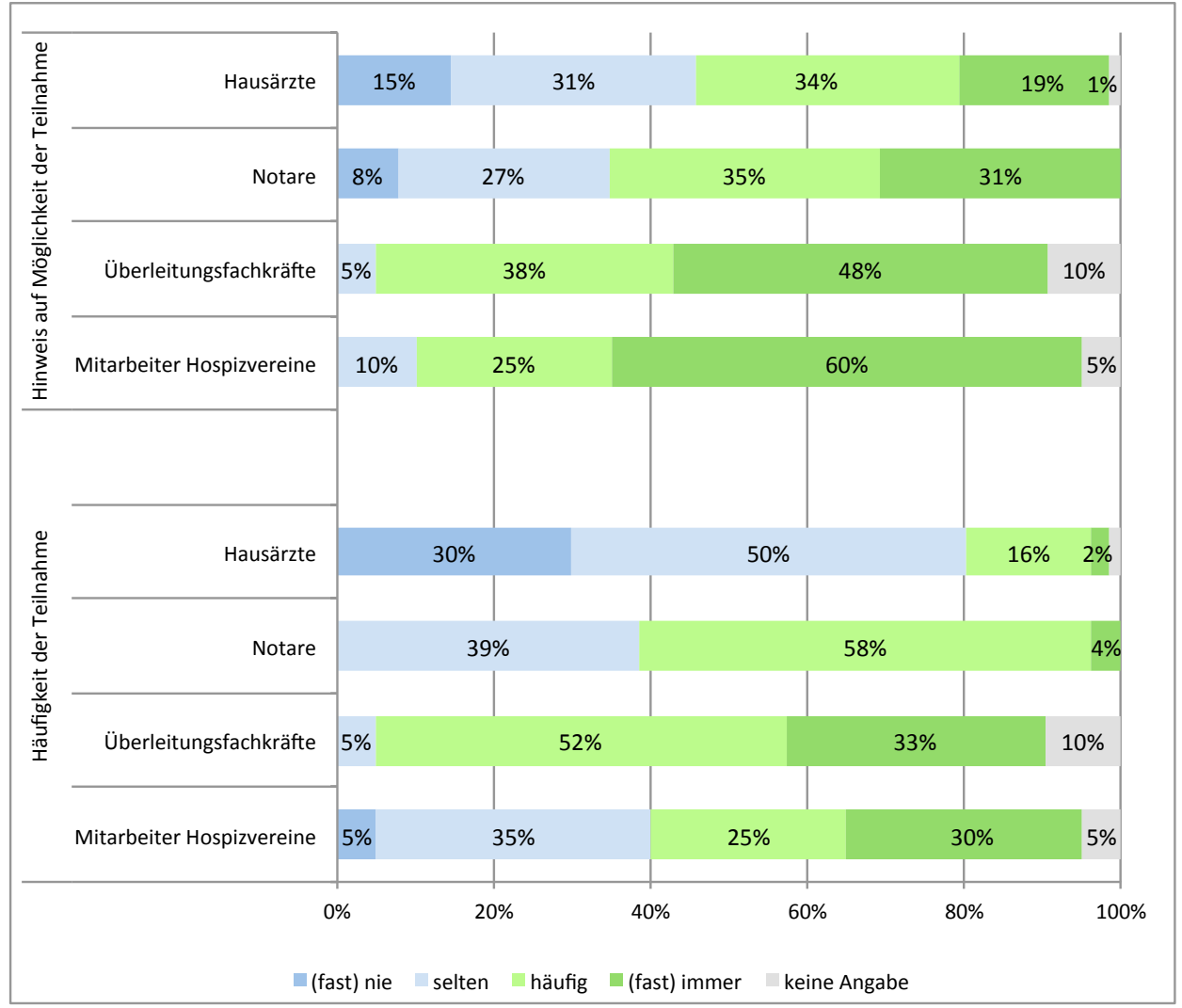


Die Mitarbeiter der Hospizvereine stellten die größte Gruppe derer, die fast immer oder häufig auf die Möglichkeit der Mitnahme des zukünftigen Vorsorgebevollmächtigten/Betreuers zur Beratung hinwiesen (h/i $85 \%$ ). So kann man davon ausgehen, dass der Einbeziehung der zukünftigen Vertreter bei den Mitarbeitern der Hospizvereine viel Wert beigemessen, und eine Beteiligung gefördert wurde. Lediglich 10\% dieser Berater fügten diesen Hinweis nur selten in die Beratung ein.

Ähnlich zeigte sich die Situation bei den Überleitungsfachkräften: Der Anteil der Berater, die fast immer die Möglichkeit der Einbeziehung erwähnten (48\%), war zwar geringer als bei den Hospizmitarbeitern (60\%). Addiert man jedoch diejenigen, die diesen Hinweis häufig geben (h 38\%) hinzu, kann man bei den Überleitungsfachkräften ebenfalls auf ein großes Interesse an der Einbeziehung der zukünftigen Vertreter schließen (h/i $86 \%)$.

Bei den Notaren und Ärzten machten, im Verhältnis zu den anderen Gruppen, relativ viele Berater selten oder fast nie auf die Einbeziehung der Vertreter aufmerksam (Notare n/s 35\%, Ärzte n/s 46\%).

Die Aussagen der Gruppen zur Häufigkeit der Teilnahme des zukünftigen Vertreters an dem Beratungsgespräch waren innerhalb und zwischen den Gruppen heterogen. Nur bei den Überleitungsfachkräften zeichnete sich eine klare Tendenz zur Teilnahme dieser Personen ab (h/i 85\%). Die Notaren gaben zwar keine klare Aussage über eine regelmäßige Teilnahme (i $4 \%$ ), jedoch berichteten $58 \%$ von einer häufigen Teilnahme der Vertreter. Dagegen kreuzte ein großer Teil dieser Berufsgruppe an, die Einbeziehung erfolge nur selten (s 39\%).

Auch die Beratungen der Mitarbeitern der Hospizvereine bezogen den zukünftigen Vertreter unterschiedlich häufig mit ein: gut die Hälfte dieser Berater führte aus, diese Personen würden häufig oder fast immer teilnehmen (h/i 55\%). Bei 40\% dieser Berater war er jedoch nur selten und bei $4 \%$ fast nie eingebunden.

Am wenigsten häufig nahmen zukünftige Vertreter an den von Ärzten durchgeführten Beratungen teil. Die Hälfte der Ärzte erklärte, die Einbeziehung sei selten, weitere $30 \%$ kreuzten an, sie erfolge fast nie. Lediglich 
bei $2 \%$ der befragten Ärzte nahm der Vertreter fast immer und bei $16 \%$ häufig an der Beratung teil.

\subsubsection{Auswirkung der Teilnahme des Vertreters}

Die Einbeziehung eines zukünftigen Vorsorgebevollmächtigten/Betreuers bewerteten alle Beratergruppen überwiegend als hilfreich (Abb.18).

Abbildung 18: Auswirkung der Einbeziehung des zukünftigen Vertreters auf den Berater

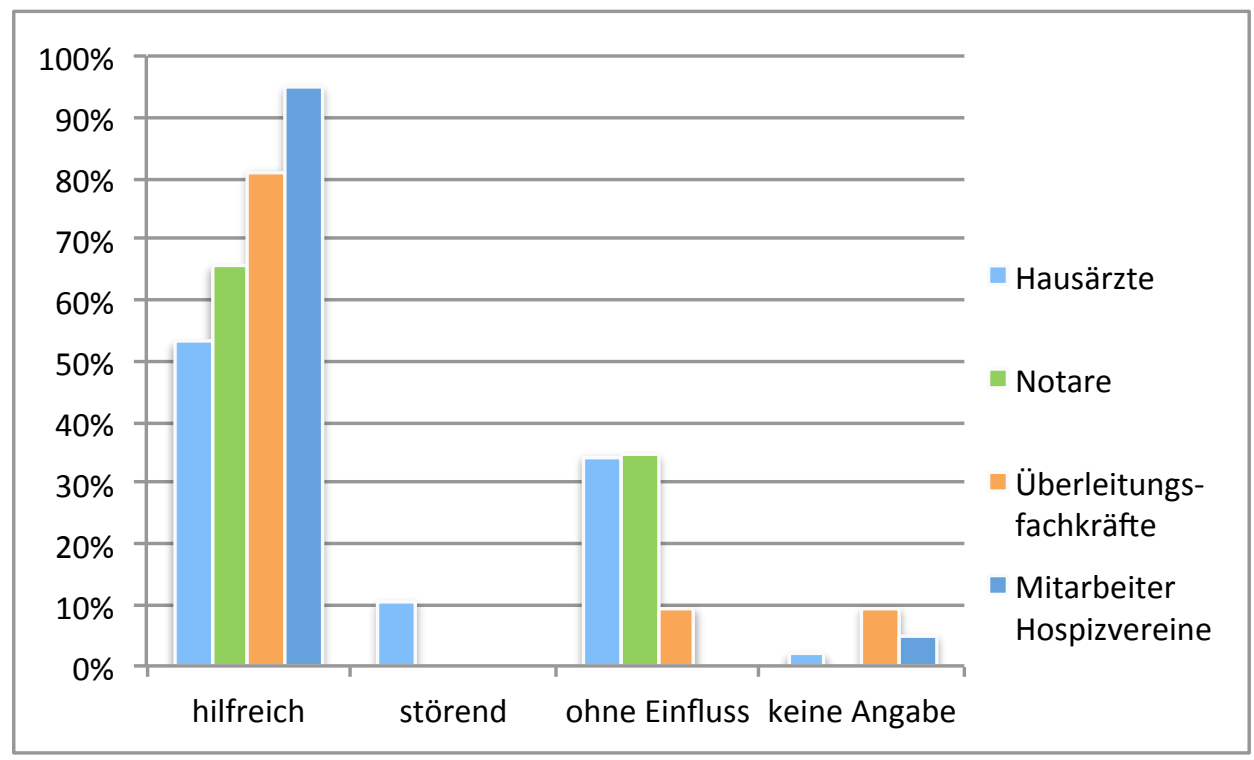

Die Mitarbeiter der Hospizvereine und die Überleitungsfachkräfte hatten anscheinend die besten Erfahrungen in diesem Bereich. Weniger positiv sahen die Ärzte die Einbeziehung: nur gut die Hälfte bezeichnete sie als hilfreich und immerhin $11 \%$ als störend. Jeweils ein Drittel der Hausärzte und Notare maß der Einbeziehung keinen Einfluss zu.

Betrachtet man bei den Ärzten, die die Teilnahme als störend empfanden, die Häufigkeit des Hinweises auf die Möglichkeit der Teilnahme zusammen mit der Häufigkeit der tatsächlichen Teilnahme, so zeigte sich folgendes Ergebnis (Tab.11): 


\begin{tabular}{|c|c|c|c|c|c|}
\hline \multirow[t]{2}{*}{\begin{tabular}{|l} 
Ärzte \\
(keine Angabe: $2 \mathrm{TN}$ ) \\
\end{tabular}} & & \multicolumn{4}{|c|}{$\begin{array}{l}\text { Wie häufig weisen Sie auf die Möglichkeit hin, einen } \\
\text { zukünftigen Vorsorgebevollmächtigten/Betreuer } \\
\text { zur Beratung mitzubringen? }\end{array}$} \\
\hline & & (fast) nie & selten & häufig & (fast immer) \\
\hline \multirow{3}{*}{$\begin{array}{l}\text { Empfinden Sie die Einbeziehung des } \\
\text { zukünftigen Vertreters für Sie } \\
\text { persönlich als eher: }\end{array}$} & hilfreich & 5 & 14 & 32 & 19 \\
\hline & störend & 4 & 9 & 1 & 0 \\
\hline & ohne Einfluss & 10 & 18 & 11 & 6 \\
\hline
\end{tabular}

Tabelle 11: Ärzte: Hinweis auf die Möglichkeit der Mitnahme des zukünftigen Vertreters - Auswirkung der Einbeziehung des zukünftigen Vertreters

Von 14 Ärzten, die eine Einbeziehung als störend empfanden, gab nur einer an, häufig auf die Möglichkeit der Einbeziehung hinzuweisen.

\begin{tabular}{|l|l|c|c|c|c|}
\hline $\begin{array}{l}\text { Ärzte } \\
\text { (keine Angabe: } 2 \text { TN) }\end{array}$ & & \multicolumn{4}{|c|}{$\begin{array}{l}\text { Wie häufig nimmt ein zukünftiger Vorsorge- } \\
\text { bevollmächtigter/Betreuer an der Beratung teil? }\end{array}$} \\
\hline \multirow{3}{*}{$\begin{array}{l}\text { Empfinden Sie die Einbeziehung des } \\
\text { zukünftigen Vertreters für Sie } \\
\text { persönlich als eher: }\end{array}$} & & (fast) nie & selten & häufig & (fast immer) \\
\cline { 2 - 7 } & hilfreich & 12 & 38 & 18 & 2 \\
\cline { 2 - 7 } & störend & 7 & 7 & 0 & 0 \\
\cline { 2 - 7 } & ohne Einfluss & 20 & 21 & 3 & 1 \\
\hline
\end{tabular}

Tabelle 12: Ärzte: Teilnahme des zukünftigen Vertreters an der Beratung - Wirkung der Einbeziehung des zukünftigen Vertreters auf den Berater

Es kreuzte auch keiner dieser 14 Ärzte an, ein zukünftiger Vertreter würde häufig oder immer an der Beratung teilnehmen. Die Einstellung oder Erfahrung der Ärzte mit der Einbeziehung des Vertreters scheint also in einer Wechselbeziehung mit der Häufigkeit der Einbeziehung des zukünftigen Vertreters zu stehen. 
3.2.15 Hinweis auf die Möglichkeit einer Organspende

Der Großteil der Berater wies (fast) nie oder selten auf die Möglichkeit einer Organspende hin (Abb.19).

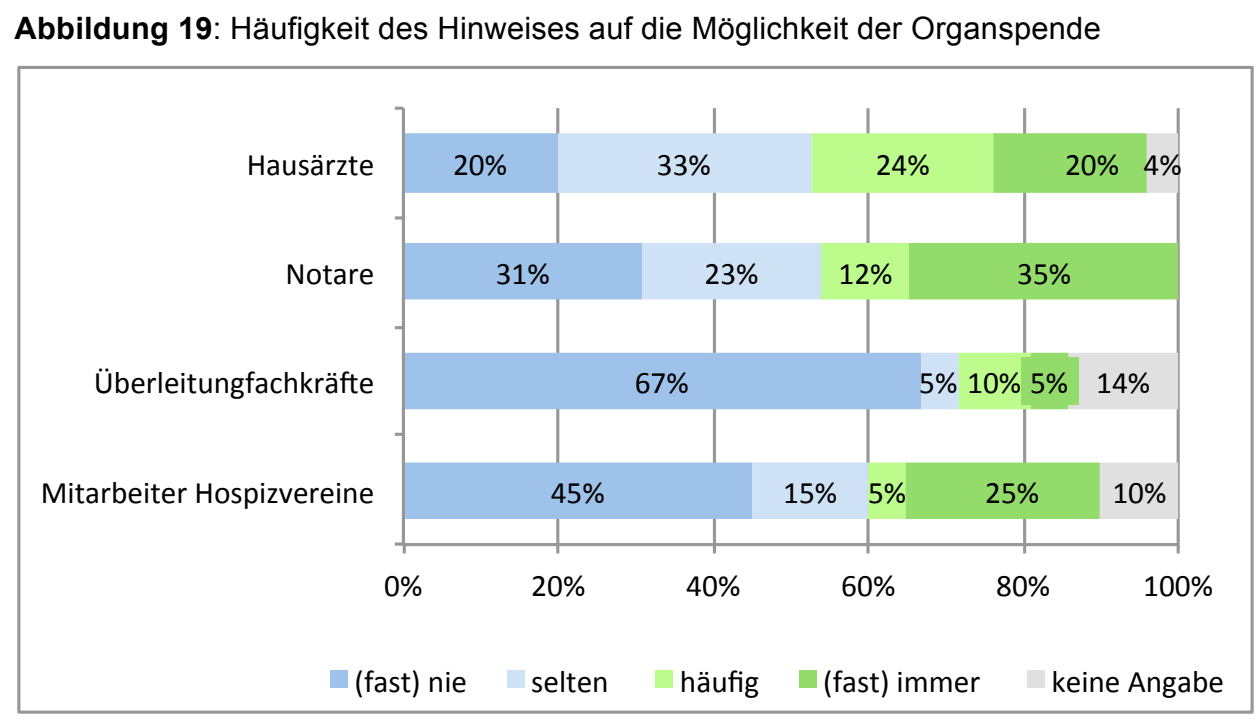

Das Thema wurde am häufigsten von den Notaren einbezogen, bei über einem Drittel dieser Berater gehörte es (fast) immer dazu. Auch bei den Hausärzten spielt es eine große Rolle. Auffällig ist, dass es bei diesen zwei Berufsgruppen jeweils zwei ähnlich große Gruppen gab: Eine Gruppe die häufig oder fast immer zu diesem Thema beriet (44\%), und eine zweite Gruppe (55\%), die es selten oder nie berücksichtigte.

Ein Viertel der Mitarbeiter der Hospizvereine hatte den Hinweis auf die Möglichkeit der Organspende fest in die Beratung etabliert (h/i 28\%), gut die Hälfte dieser Berater jedoch selten bis gar nicht (n/s 60\%).

Bei den Überleitungsfachkräften wurde er vom Großteil der Berater (fast) nie gegeben (n 67\%, s 5\%). 


\subsubsection{Themen im Hinblick auf eine Organspende}

Die mit der Organspende zusammenhängende inhaltliche Thematik wurde von den Beratern wenig häufig angesprochen (Abb. 20).

Abbildung 20: Themen, die im Hinblick auf eine Organspende angesprochen werden

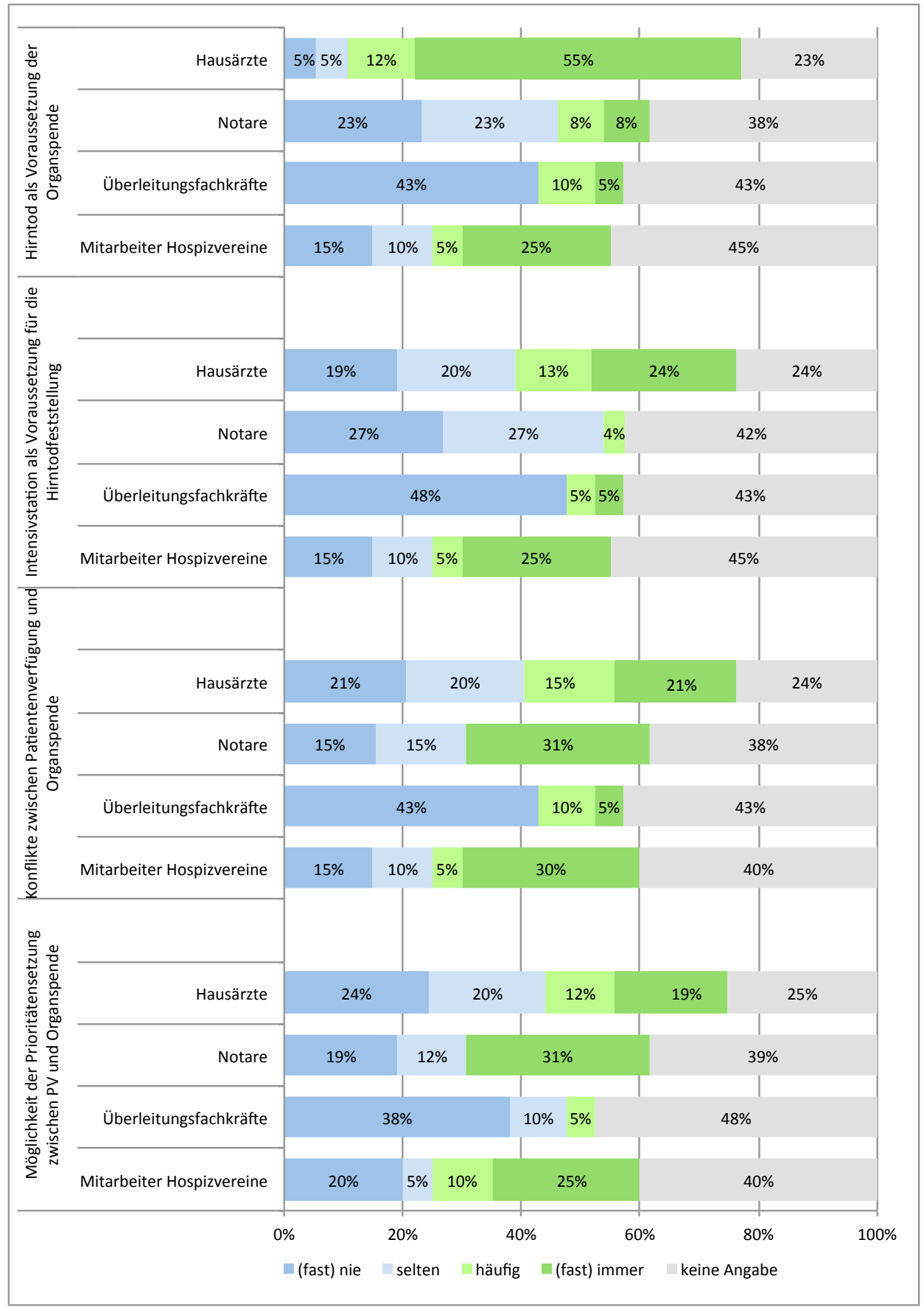


Am häufigsten sind inhaltliche Themen der Organspende von den Hausärzten aufgegriffen worden. Über ein Drittel bezog alle angebotenen Themen häufig oder fast immer ein, beim Thema „Hirntod als Voraussetzung für Organspende“ waren es zwei Drittel der Ärzte.

Die Notare bildeten zwar die Gruppe, die das Thema Organspende allgemein am häufigsten ansprach, bei der inhaltlichen Aufbereitung spielten jedoch nur die zwei Fragen nach der Vereinbarkeit von Patientenverfügung und Organspende bei einem Drittel dieser Berater eine Rolle.

Bei den Überleitungsfachkräften schien es kaum eine inhaltliche Beratung zur Organspende zu geben.

Rund ein Drittel der Mitarbeiter der Hospizvereine bezog häufig oder fast immer alle angebotenen Themen ein. 


\subsection{Umfeld der Beratung}

3.3.1 Einbeziehung der Berater in die Entscheidungsfindung bei Einwilligungsunfähigkeit der Beratenen

Durch das Beratungsgespräch erhalten viele Berater einen Einblick in die Wünsche und Werte des Ratsuchenden. In die Ermittlung des Patientenwillens bei dessen Einwilligungsunfähigkeit wurden die Gruppen jedoch sehr unterschiedlich einbezogen (Abb. 21).

Abbildung 21: Beteiligung bei der Ermittlung des Patientenwillens bei Einwilligungsunfähigkeit

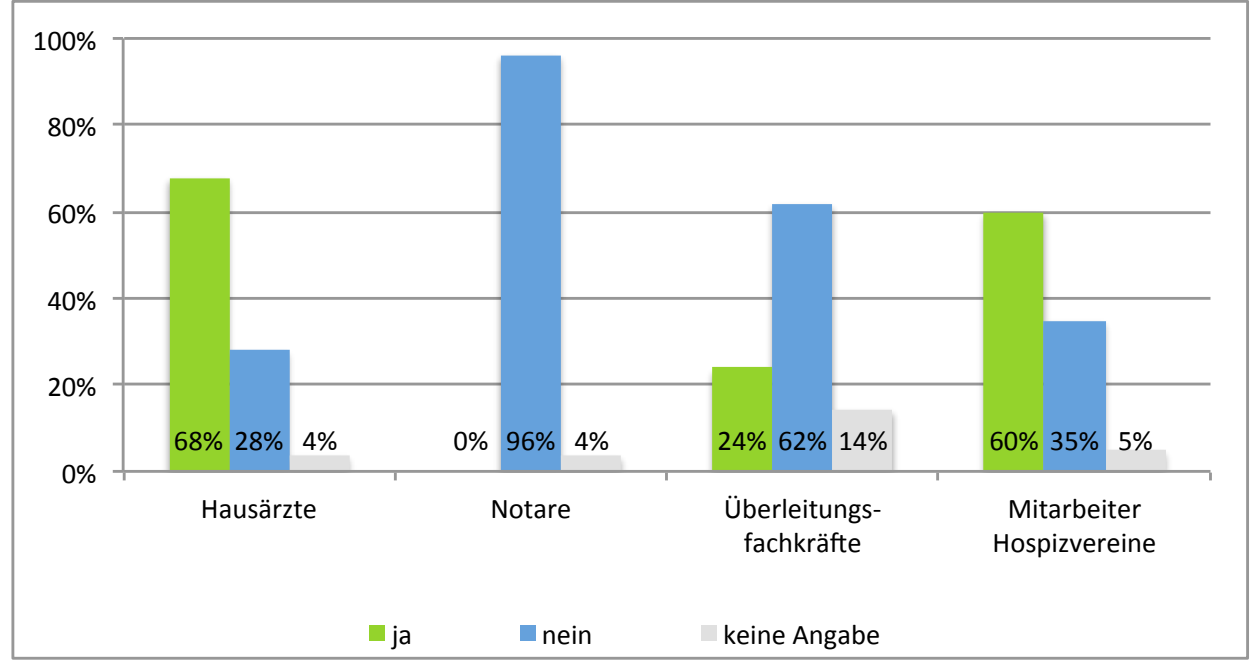

Eine Beteiligung an der Entscheidungsfindung wurde am häufigsten von Hausärzten und Mitarbeitern der Hospizvereine angegeben. Über 60\% dieser Berater führte aus, bei der Entscheidungsfindung zugezogen zu werden. Ungefähr ein weiteres Drittel dieser Berufsgruppen (Mitarbeiter Hospizvereine 35\%, Ärzte 28\%) kreuzte an, nicht an der Entscheidungsfindung beteiligt zu werden.

Bei den Überleitungsfachkräften sah sich knapp ein Viertel (24\%) in die Entscheidungsprozesse involviert. Der weitaus größere Teil der Gruppe (62\%) gab an, nicht mit dieser Aufgabe befasst zu werden.

Notare wurden überhaupt nicht in dieses Entscheidungsfeld involviert.

Das Wissen der Berater über die Wünsche des Patienten wurde demnach vielfach nicht in die Entscheidungsfindung einbezogen. 
3.3.2 Häufigkeit des Verweises an andere Berufsgruppen zur ergänzenden Beratung

Um zu erfahren, wie offen die Berater für die Einbeziehung anderer Berufsgruppen in die Beratung und eine Vernetzung mit diesen sind, wurde gefragt, wie häufig sie zur ergänzenden Beratung an andere Berufsgruppen verweisen.

Mit Ausnahme der Überleitungsfachkräfte zeichneten sich bei den Teilnehmern der Beratergruppen zwei Verfahrensweisen im Hinblick auf die Verweisung an andere Berufsgruppen ab (Abb. 22).

Abbildung 22: Häufigkeit des Verweises an andere Berufsgruppen zur ergänzenden Beratung

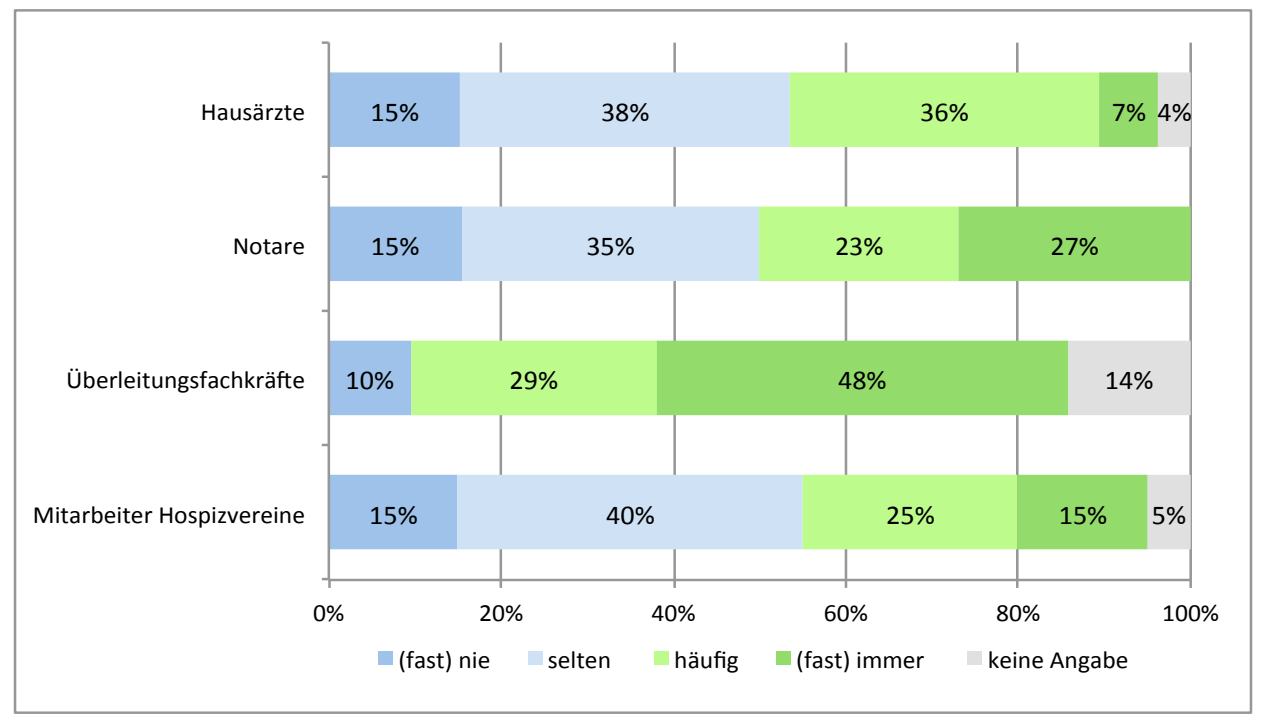

Knapp die Hälfte verwies häufig oder immer (h/i 40-50\%) und die etwas größere Hälfte selten oder fast nie (n/s 50-55\%).

Die Überleitungsfachkräfte dagegen kreuzten zu mehr als drei Viertel an, häufig oder fast immer an andere Berufsgruppen zu überweisen (h/i 77\%). Sie stellten nach diesen Angaben diejenige Berufsgruppe, die am häufigsten an andere verweist und somit die größte Offenheit zeigte. 
3.3.3 Berufsgruppen, an die zur ergänzenden Beratung verwiesen wird

Um Hinweise dafür zu erhalten, welche Verbindungen zwischen den Beratergruppen bestehen und welche fachlichen Kompetenzen sich aus Sicht der Berater ergänzen, wurde den Beratern eine Liste mit verschiedenen Professionen zur Verfügung gestellt.

Die Berufsgruppe, an die von anderen Beratergruppen am häufigsten verwiesen wurde, sind die Ärzte $(65-89 \%)$, gefolgt von den Hospizvereinen $(30-71 \%)$ und an dritter Stelle den Betreuungsvereinen (12-40\%). Notare wurden deutlich weniger einbezogen und die Pflege noch weniger (Abb. 23).

Abbildung 23: Berufsgruppen, an die zur ergänzenden Beratung verwiesen wird

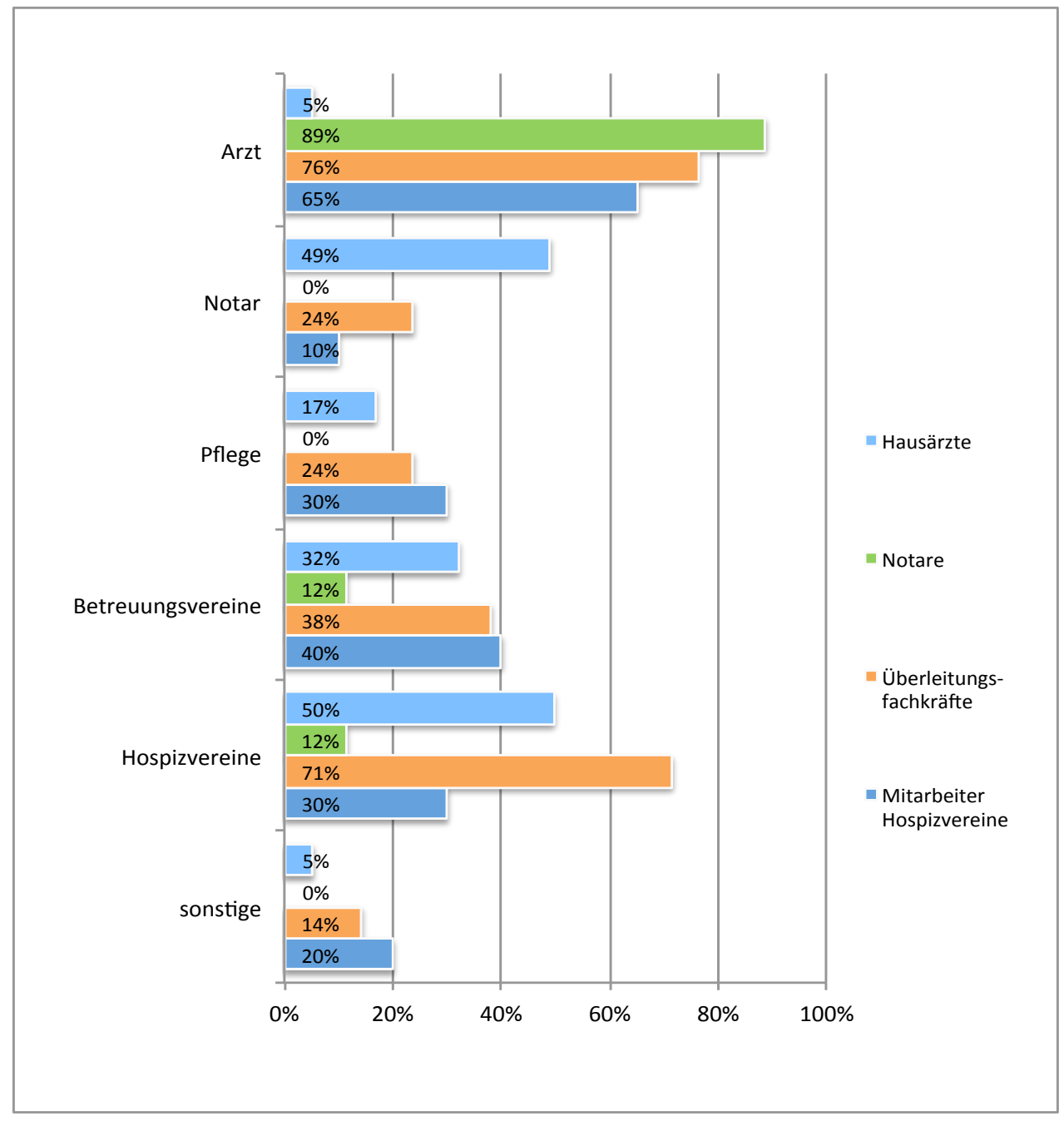

Mehrfachnennungen möglich 
Die Notare verwiesen fast alle an Ärzte (89\%) und nur zu einem kleinen Teil auch an Betreuungs- und Hospizvereine (je 12\%). Der Pflege und den „Sonstigen“ kam hier keine Relevanz zu.

Jeder zweite Arzt bezieht Notare und Hospizvereine ein, ein Drittel auch Betreuungsvereine und weniger als ein Fünftel die Pflege.

Etwa je drei Viertel der Überleitungsfachkräfte benannten Ärzte und Hospizvereine. An zweiter Stelle wurden Betreuungsvereine aufgeführt. Notare und die Pflege wurden nur von jedem fünften dieser Berater als Partner, an den verwiesen wird, angekreuzt.

Knapp zwei Drittel der Mitarbeiter der Hospizvereine verwiesen auf eine ergänzende Beratung durch Ärzte (65\%). An zweiter Stelle folgten die Betreuungsvereine (40\%) und danach die Pflege (30\%). Von eher geringerer Bedeutung blieb die Verbindung zu den Notaren (10\%). 
3.3.4 Wunsch nach verstärkter Zusammenarbeit mit bestimmten Berufsgruppen

Die Wünsche der Berater nach verstärkter Zusammenarbeit oder einem verstärkten Austausch mit anderen Beratergruppen richteten sich am häufigsten auf die Gruppe der Ärzte (62-80\%) (Abb.24).

Abbildung 24: Wunsch nach verstärkter Zusammenarbeit/Austausch mit folgenden Berufsgruppen

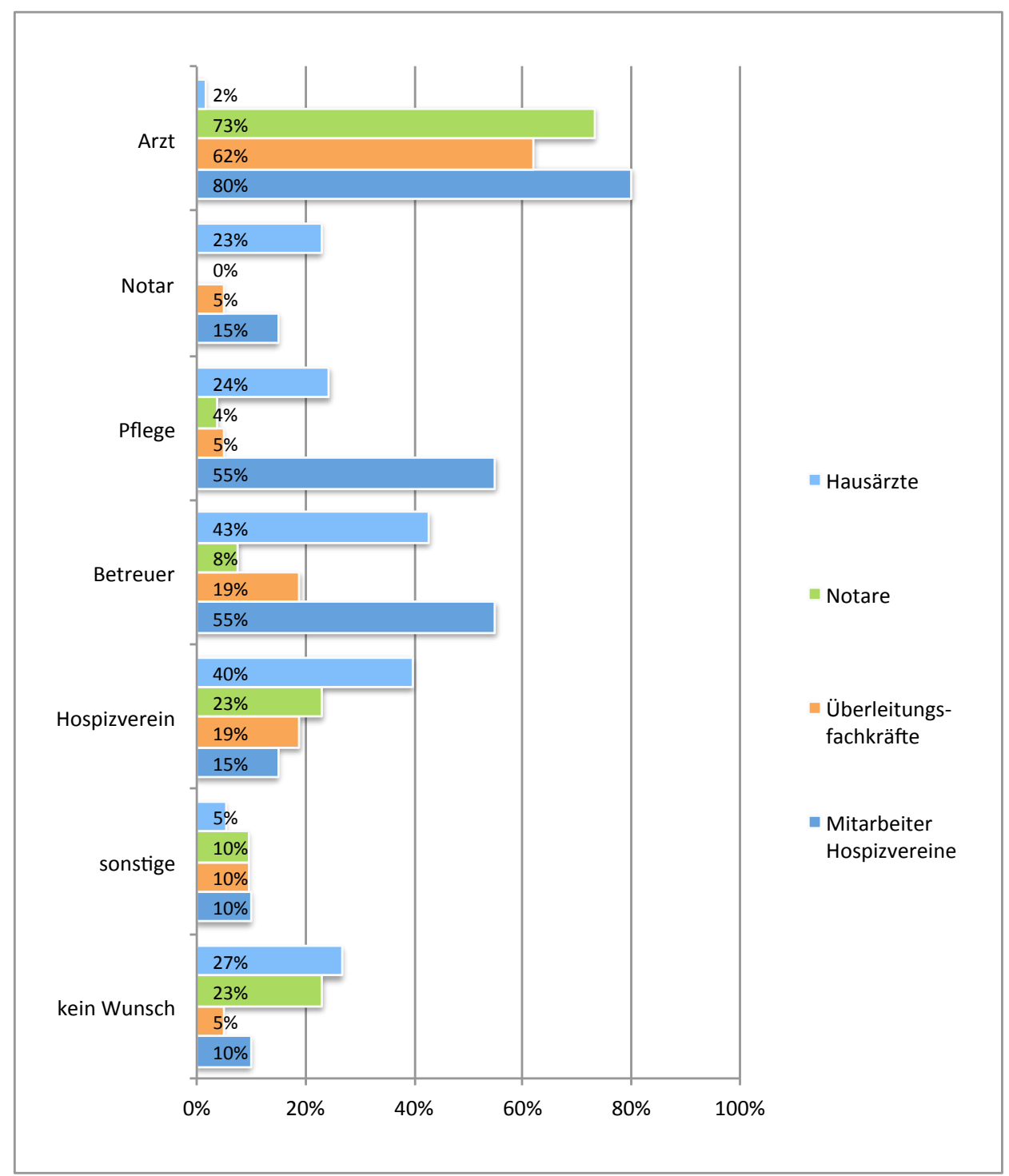

Mehrfachnennungen möglich 
Mit großem Abstand dazu wurden die Betreuungsvereine (8-55\%) und die Hospizvereine genannt (15-40\%).

Die Ärzte wünschten sich hauptsächlich eine verstärkte Zusammenarbeit mit den Betreuern (43\%) und den Hospizvereinen (40\%). Über ein Viertel sah allerdings keinen Bedarf an einer Intensivierung des Verhältnisses zu anderen Berufsgruppen (27\%).

Drei Viertel der Notare benannten in diesem Zusammenhang die Ärzte (73\%), knapp ein Viertel die Hospizvereine (23\%). Ein weiteres knappes Viertel sah keinen Bedarf an einer verstärkten Zusammenarbeit oder einem verstärkten Austausch (23\%).

Bei den Überleitungsfachkräften lag der Bedarf bei weit über der Hälfte der Teilnehmer im Bereich der Zusammenarbeit mit den Ärzten (62\%). Je ein Fünftel sieht inn im Hinblick auf Betreuer (19\%) und Hospizvereine $(19 \%)$ als gegeben. Notare und Pflege wurden kaum als Wunschpartner gewählt (je 5\%).

3.3.5 Bereiche, in denen mehr Austausch/Information gewünscht wird

Die Bereiche, in denen mehr Information/Austausch/Zusammenarbeit gewünscht wurde, waren je nach Beratergruppe sehr unterschiedlich (Abb.25). 
Abbildung 25: Bereiche, in denen mehr Austausch/Information gewünscht wird

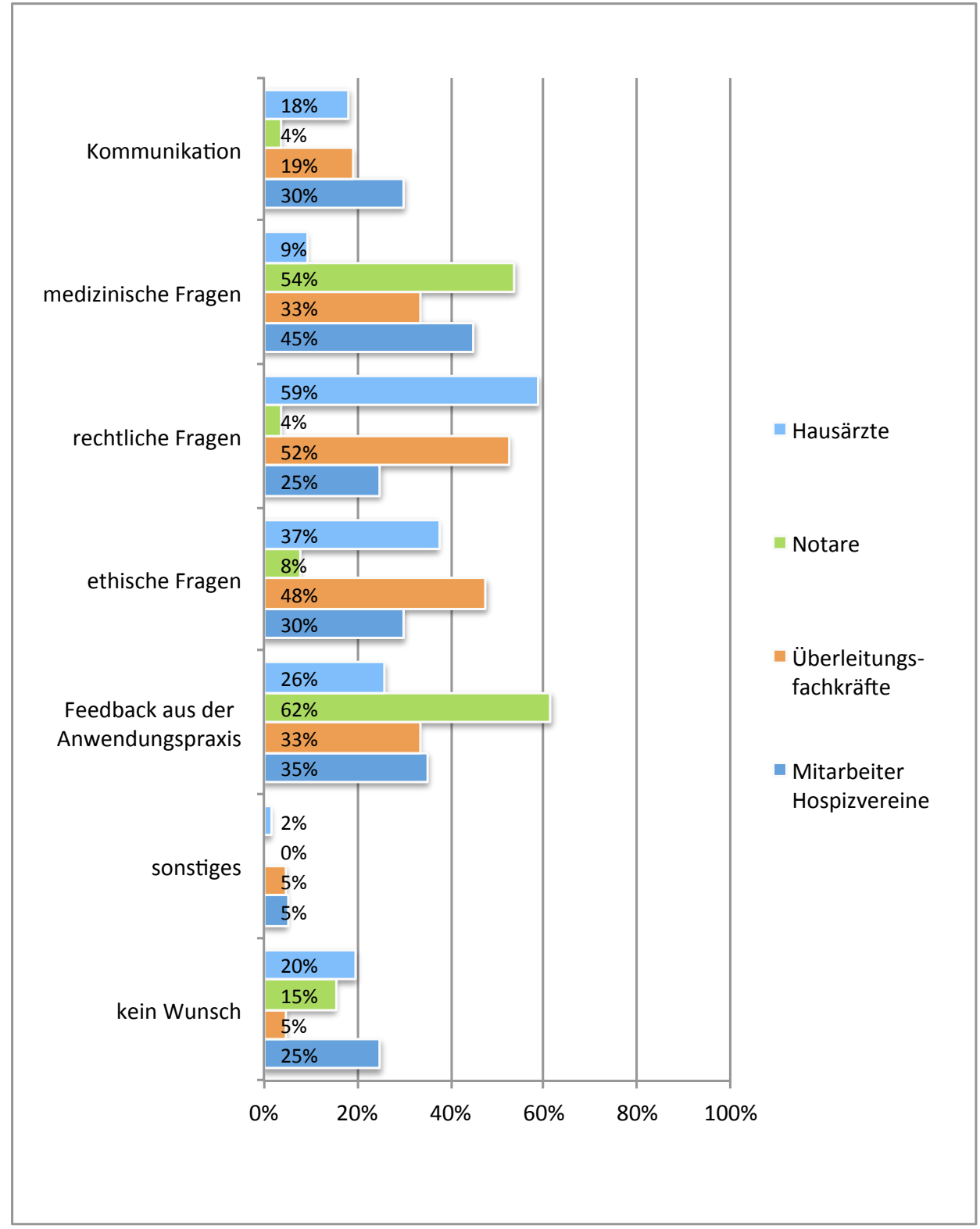

Mehrfachnennungen möglich

Über die Hälfte der Ärzte benannte primär rechtliche Fragen (59\%), gefolgt von ethischen Fragen (37\%) und einem Feedback aus der Anwendungspraxis der Patientenverfügung (26\%).

Bei den Notaren lag das Hauptinteresse auf einem Feedback aus der Anwendungspraxis (62\%) und medizinischen Fragen (54\%). Alle anderen Bereiche waren kaum relevant. 
Die Überleitungsfachkräfte kreuzten am häufigsten das Feld „Rechtliche Fragen" an (52\%). Fast jeder zweite Berater dieser Gruppe wünschte sich daneben mehr Austausch/Information im Bereich der ethischen Fragen $(48 \%)$.

Der Schwerpunkt der Wünsche der Mitarbeiter der Hospizvereine befand sich im Bereich „Medizinische Fragen“ (45\%), gefolgt von einem „Feedback aus der Praxis“ (35\%) sowie Fragen der Ethik (30\%) und der Kommunikation (30\%).

Zwischen 5-25\% der Berater der verschiedenen Gruppen hatten keine Wünsche an die Verbesserung des Austausches, der Zusammenarbeit oder der Information.

3.3.6 Gewünschte Verbesserung des Austausches, der Zusammenarbeit, der Information

Alle Berufsgruppen, mit Ausnahme der Notare, wünschten sich hauptsächlich eine Verbesserung des Austausches/der Zusammenarbeit/der Information durch Zusammenarbeit im Einzelfall (Abb.26). 
Abbildung 26: Gewünschte Verbesserung des Austausches/der Zusammenarbeit/der Information

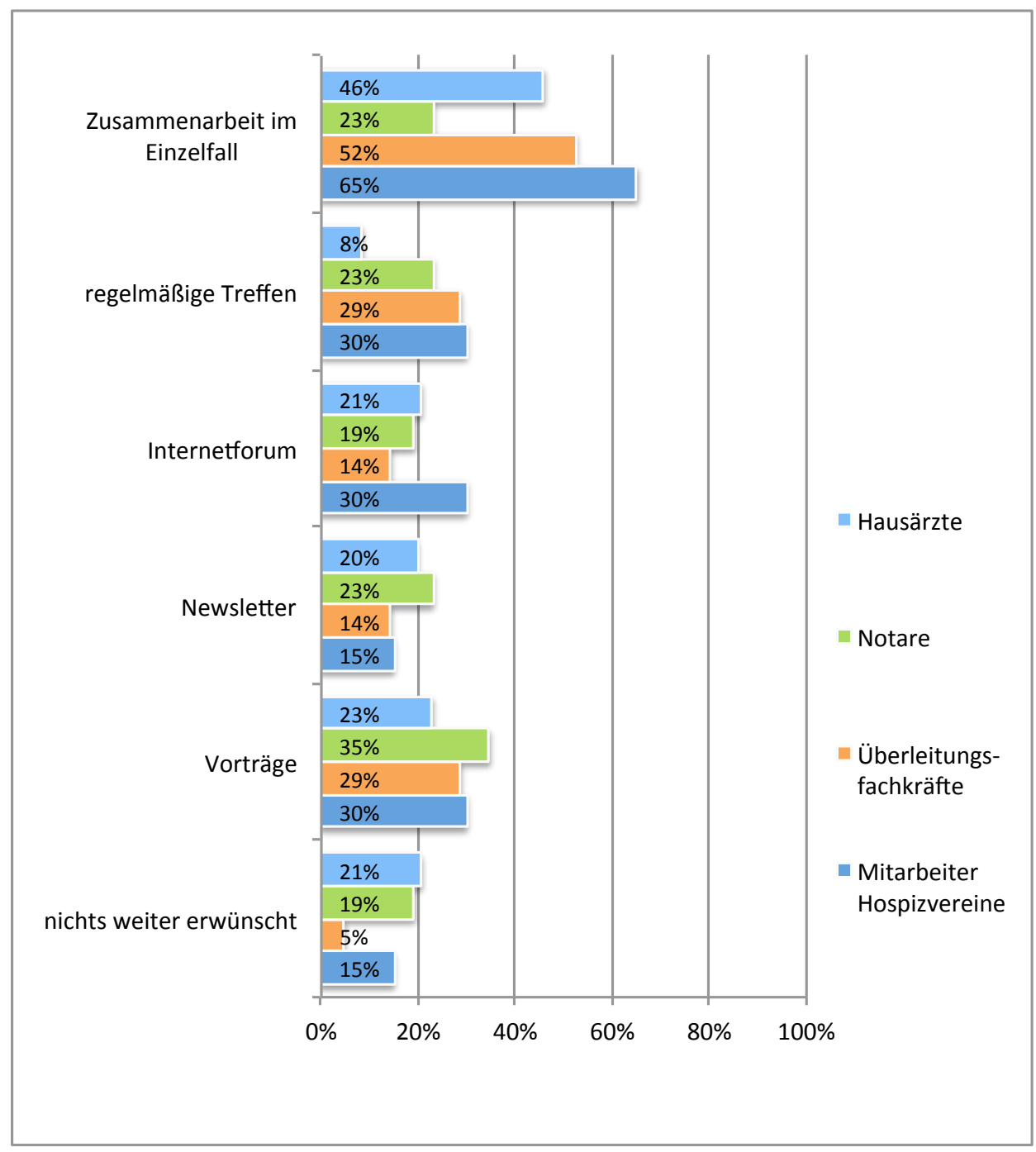

Mehrfachnennungen möglich

Die Notare wählten am häufigsten „Vorträge“ als Form der Verbesserung. Von den anderen Gruppen wurden „Vorträge“ am zweithäufigsten angekreuzt. Mit Ausnahme der Ärzte bezeichneten die Teilnehmer „regelmäßige Treffen" als dritte Möglichkeit der Verbesserung. Am vierthäufigsten wählten sie ein „Internetforum“ und an letzter Stelle ein „Newsletter“. Zwischen $5-21 \%$ der Befragten gaben an, nichts weiter zu wünschen. 
3.3.7 Routinemäßiges Angebot einer Beratung zur Patientenverfügung Insgesamt betrachtet hielt die Mehrheit der Berater ein routinemäßiges Angebot einer Beratung zur Patientenverfügung ab einem bestimmten Alter für sinnvoll (57-79\%).

Abbildung 27: Routinemäßiges Beratungsangebot ab einem bestimmten Alter sinnvoll

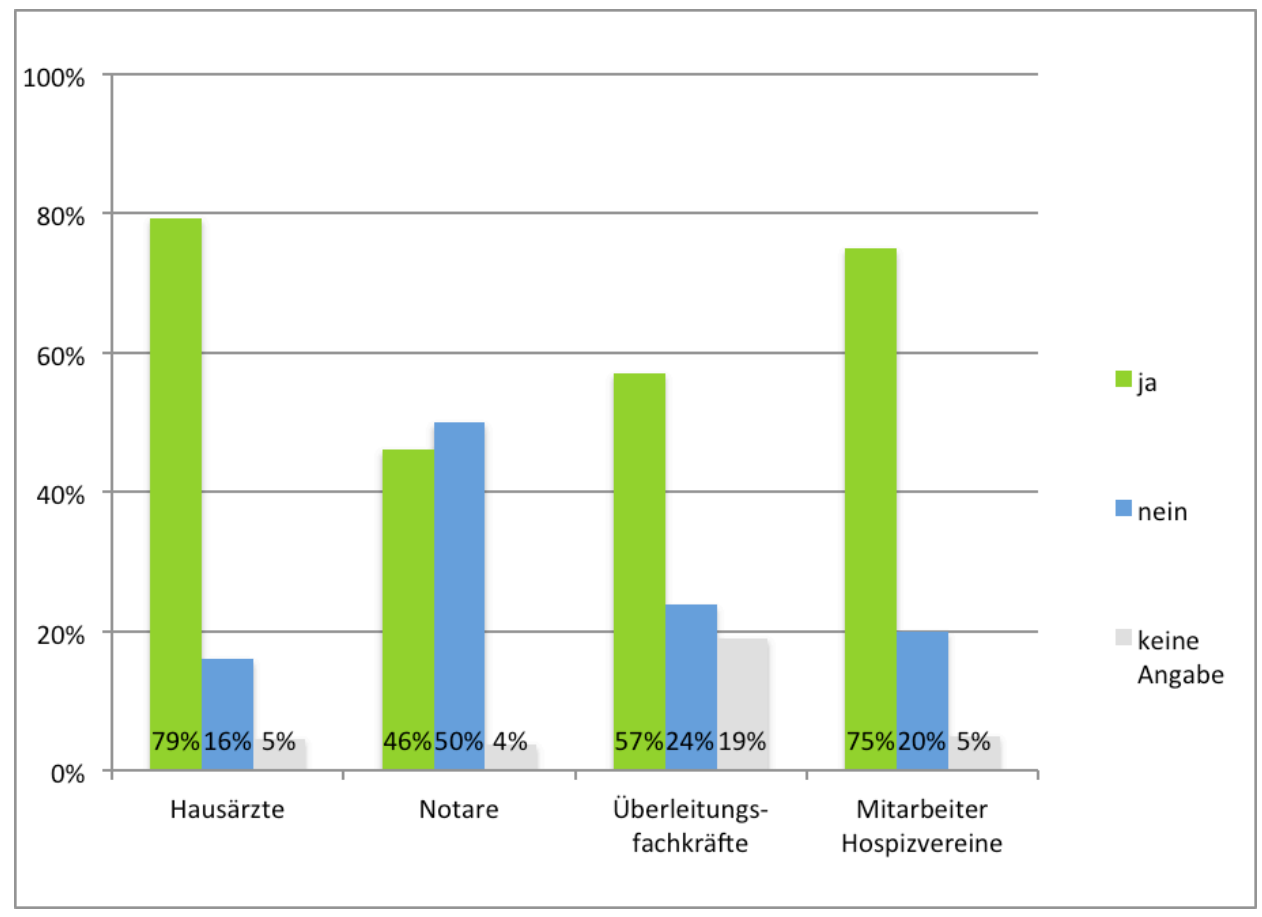

Lediglich bei den Notaren erklärte eine knappe Mehrheit (50\%) ein derartiges Angebot nicht für sinnvoll.

Bei Hausärzten und Hospizvereinen lag die Zustimmung am höchsten. 


\subsubsection{Erwerb der Kenntnisse für die Beratung}

Die Teilnehmer erarbeiten sich die für die Beratung benötigten Kenntnisse auf unterschiedlichen Wegen (Abb.28).

Abbildung 28: Erwerb der Kenntnisse für die Beratung

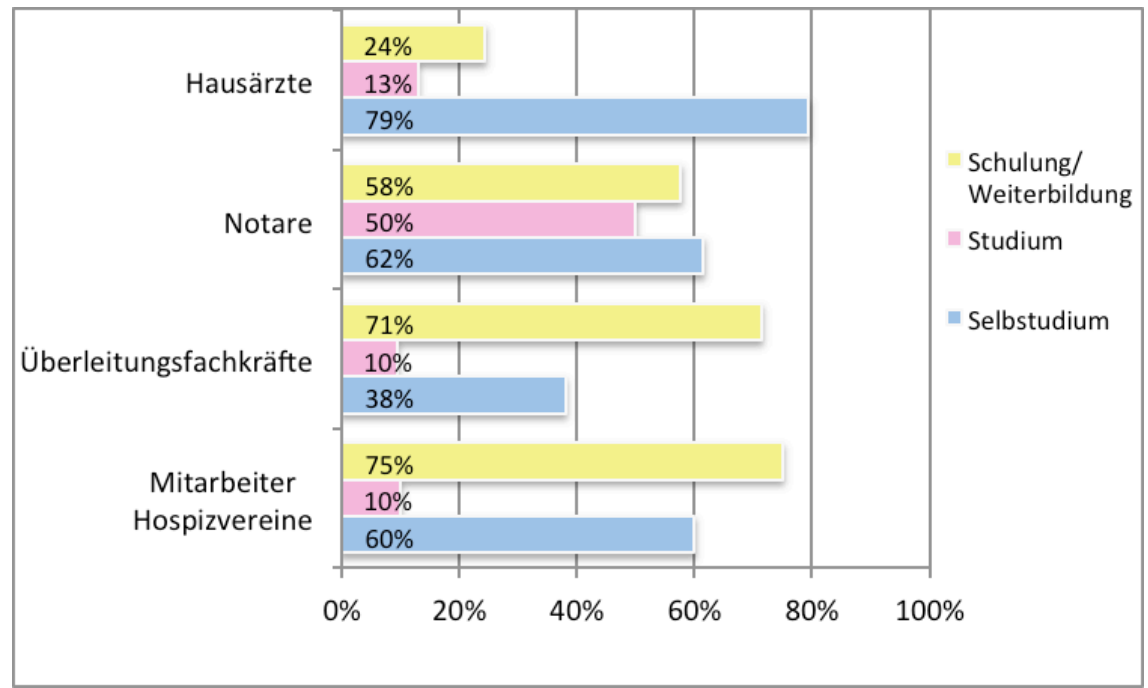

Mehrfachnennungen möglich

Ärzte und Notare erwarben ihre Kenntnisse überwiegend durch Selbststudium. Bei den Ärzten gaben weniger als ein Viertel an, an einer Schulung oder Weiterbildung zu diesem Thema teilgenommen zu haben. Der Anteil der geschulten Berater lag bei den Notaren dagegen mit $58 \%$ erheblich höher.

Überleitungsfachkräfte und Mitarbeiter von Hospizvereinen bezogen ihre Kenntnisse primär aus Schulungen und Weiterbildungen $(71 \%, 75 \%)$ und erst an zweiter Stelle durch Selbststudium. Ein Studium hatte in diesem Zusammenhang lediglich bei den Notaren eine größere Relevanz für die Beratung (50\%). 


\subsubsection{Schulung anhand von Formularen}

Die Schulungen zur Beratung zur Patientenverfügung erfolgten unterschiedlich häufig anhand bestimmter Formulare (Abb.29).

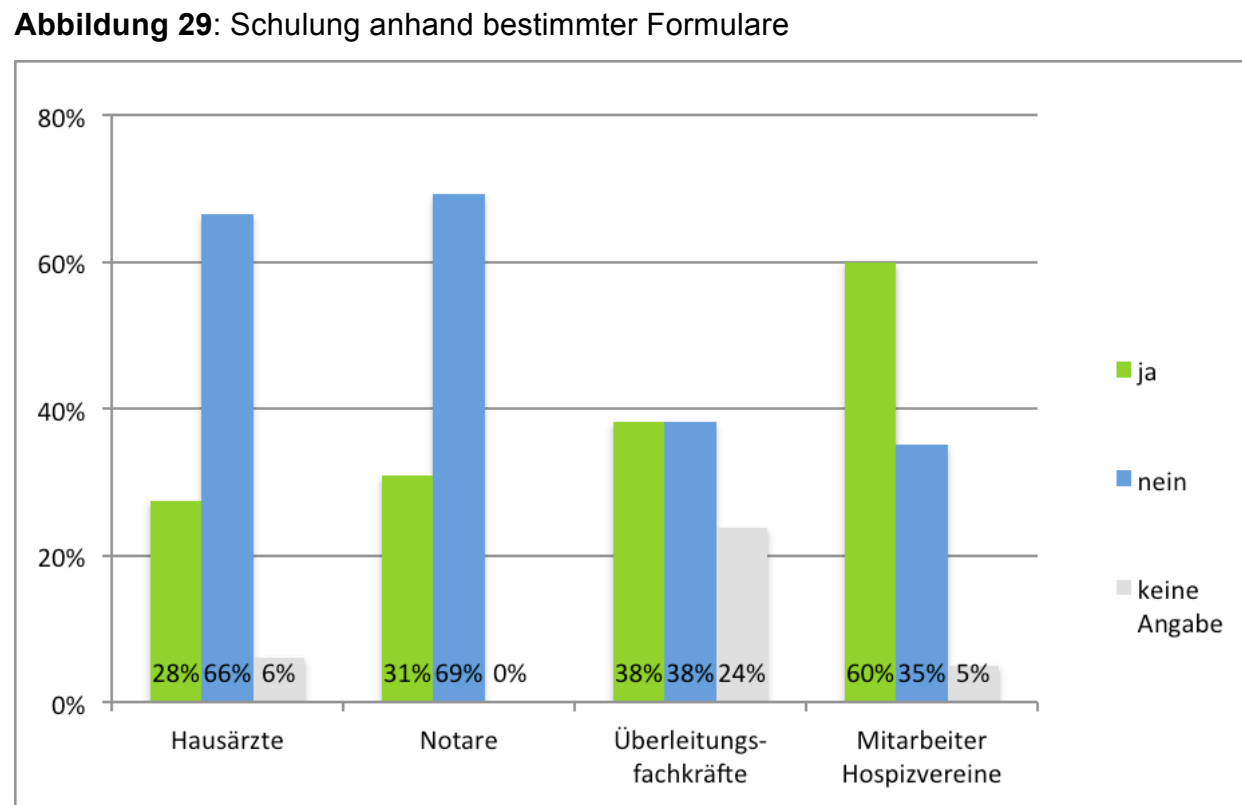

Hausärzte und Notare wurden zu zwei Dritteln nicht anhand bestimmter Formularen geschult.

Die Antworten der Überleitungsfachkräfte zeigte eine Aufteilung in zwei gleich große Gruppen.

Die Mitarbeiter der Hospizvereine bejahten überwiegend (60\%), anhand bestimmter Formulare ausgebildet worden zu sein. 


\subsubsection{Benennung der Schulungsformulare}

Die in den Schulungen/Weiterbildungen verwendeten Formulare waren nach Angaben der Berater unterschiedlich (Abb.30).

Abbildung 30: verwendete Schulungsformulare

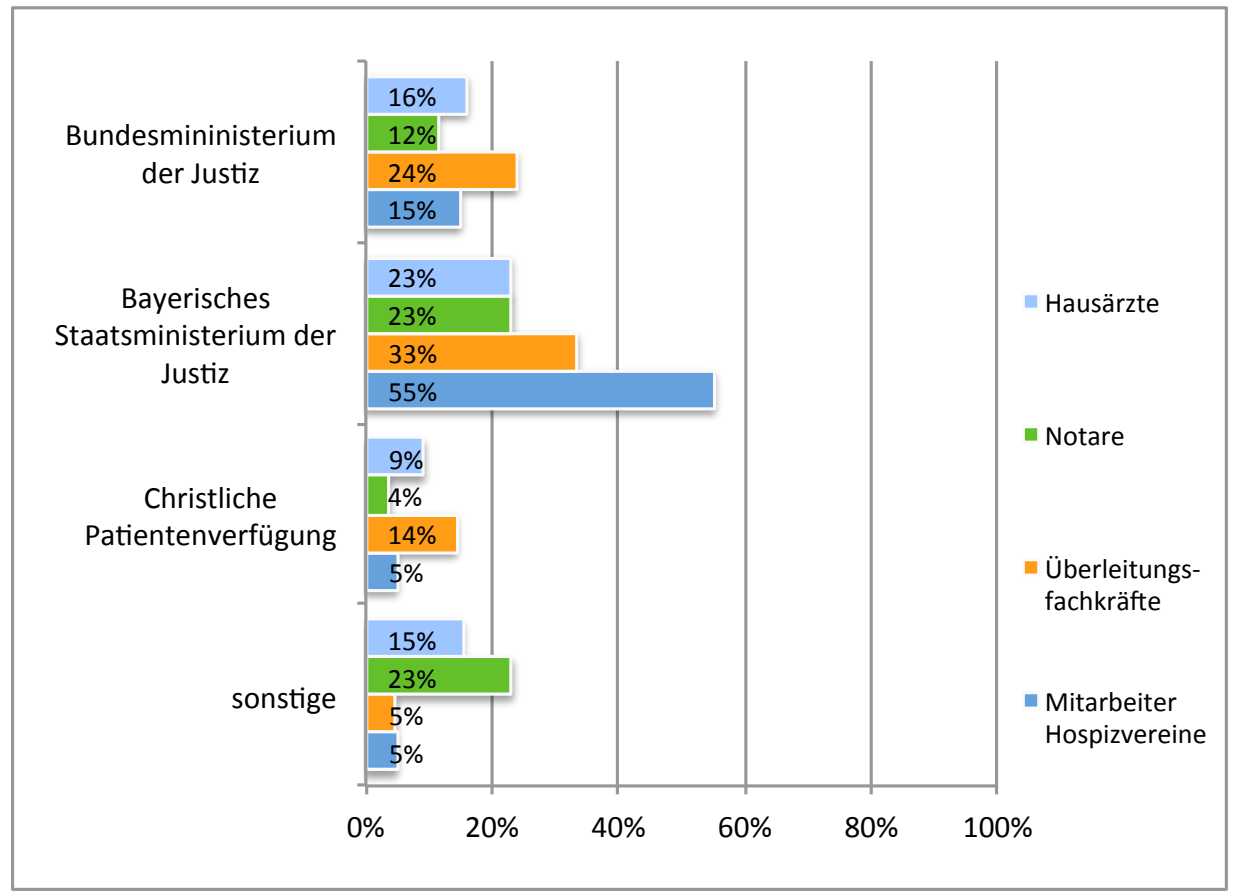

Mehrfachangaben möglich

Das Formular des Bayerischen Ministeriums der Justiz wurde bei den Schulungen am häufigsten verwendet. Bei allen Berufsgruppen kreuzte mindestens ein knappes Viertel der Befragten diese Option an. Bei der Auswertung der unter "Sonstige" durch die Befragten benannten Formulare fanden sich etliche Formulierungen, die vermuten lassen, dass auch hier das Formular des Bayerischen Ministeriums der Justiz gemeint sein soll, so dass sich die Bedeutung dieses Textes noch einmal erhöhen würde.

An zweiter Stelle wurden die Textbausteine des Bundesjustizministeriums genannt. Die christliche Patientenverfügung schien nur bei der Schulung der Überleitungsfachkräfte und einigen Hausärzten größere Relevanz zu besitzen. 


\subsubsection{Kostenpflicht der Beratung}

Die Beratung zur Patientenverfügung bringt überwiegend keine finanzielle Belastung für den Ratsuchenden mit sich (Abb.31).

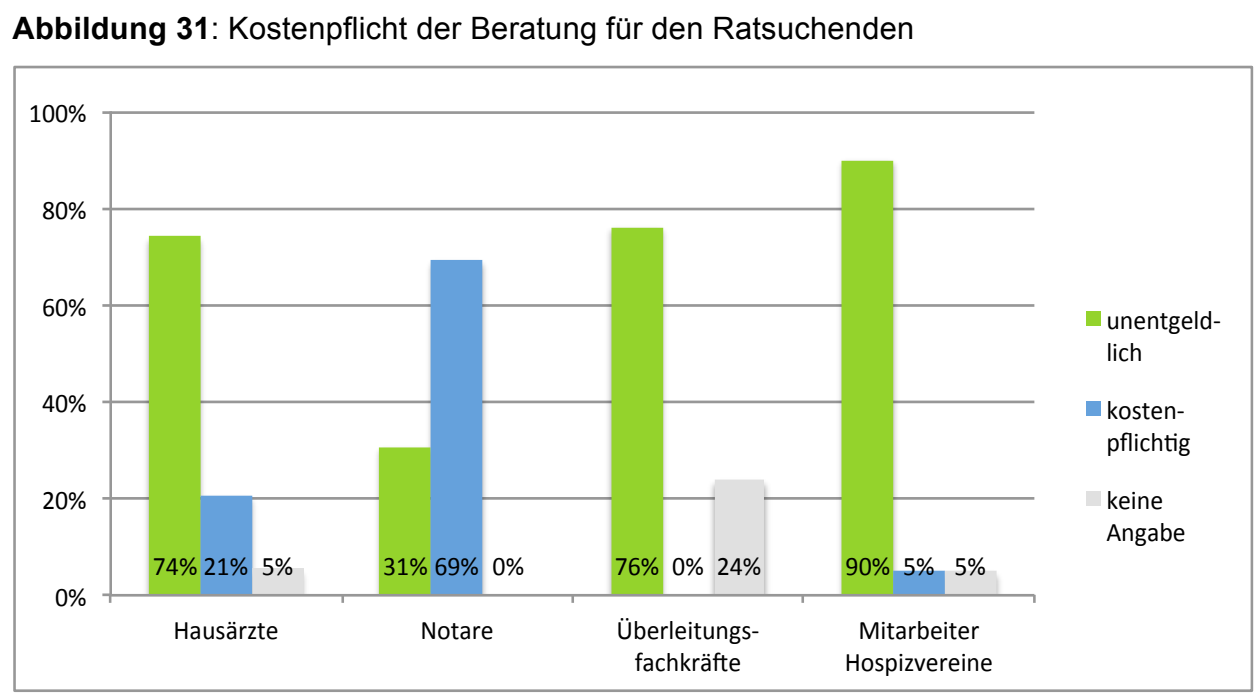

Die Beratung durch die Teilnehmer der Befragung erfolgte, mit Ausnahme der Notare, weit überwiegend unentgeltlich (>74\%).

Die Beratung durch Hausärzte war bei drei Vierteln der Befragten dieser Berufsgruppe für den Ratsuchenden unentgeltlich und nur bei einem Fünftel entgeltlich.

Auch 30\% der Notare stellten dem Ratsuchenden keine diesbezüglichen Beratungskosten in Rechnung.

Die Überleitungsfachkräfte gaben zu drei Vierteln an, ihre Beratung sei für den Ratsuchenden unentgeltlich. Die restlichen $24 \%$ der Berater ließen diese Frage unbeantwortet.

Die Mitarbeiter der Hospizvereine berieten hauptsächlich unentgeltlich $(90 \%)$ und nur selten entgeltlich $(5 \%)$. 


\subsubsection{Vergütung für den Berater}

Die Beratung erfolgte weit überwiegend ohne Vergütung für die Berater (Abb.32).

Abbildung 32: Erhalt einer Vergütung

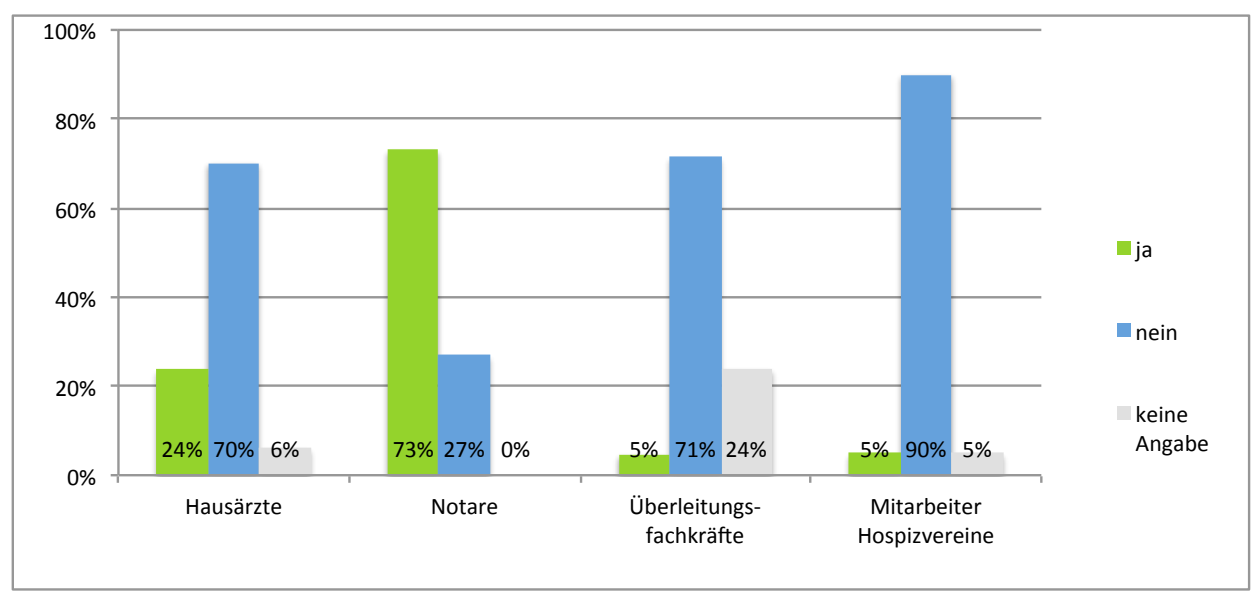

Mit Ausnahme der Notare gab die Mehrheit der Mitglieder der befragten Gruppen an, keine Vergütung für die Beratung zu erhalten (70 - 90\%).

Bei den Notaren erklärte ein Viertel der Befragten (27\%), keine Beratungsvergütung zu bekommen.

\subsubsection{Angemessenheit der Vergütung}

Mit Ausnahme der Notare hielt die Mehrheit der Befragten, die eine Vergütung erhielten, diese nicht für angemessen (Abb.33).

Abbildung 33: Einschätzung der Angemessenheit der Vergütung durch Berater, die eine Vergütung erhalten

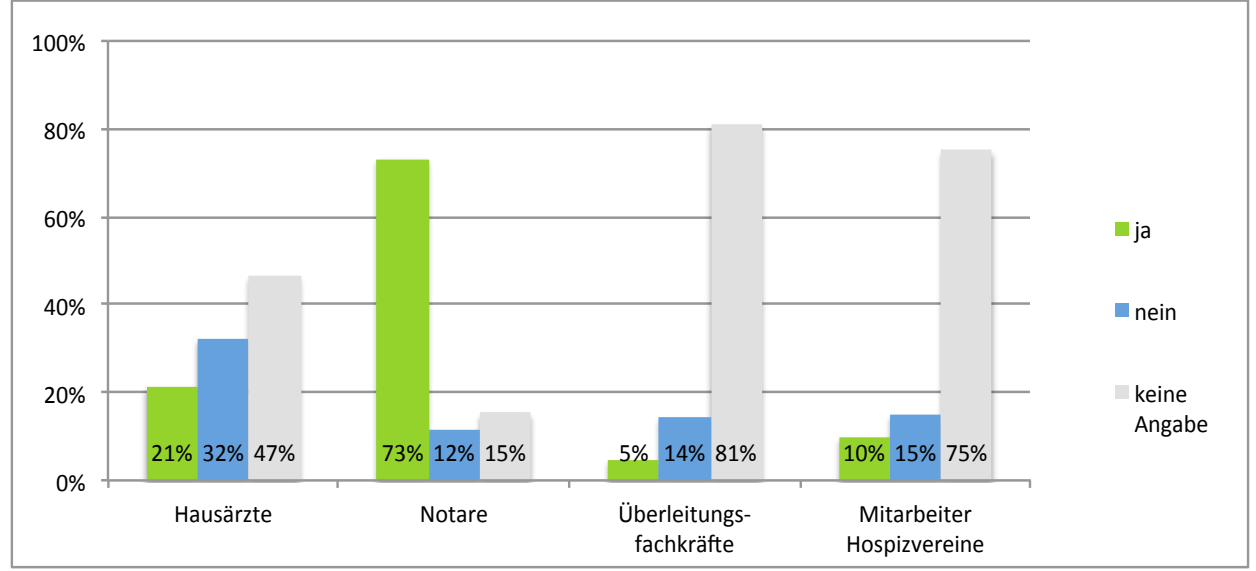




\subsubsection{Angemessene Höhe der Vergütung}

Von 104 Beratern, die Angaben zur Höhe einer angemessenen Vergütung machten, sprachen sich 35 Personen für eine Vergütung in Höhe von bis zu 49 Euro pro Beratung aus. 50 Personen wünschten eine Vergütung von 50-99 Euro für eine Beratung (Tab.13).

\begin{tabular}{|c|c|c|c|c|}
\hline angemessene Höhe der Vergütung & $\begin{array}{c}\text { Hausärzte } \\
(\mathrm{n}=131)\end{array}$ & $\begin{array}{c}\text { Notare } \\
(\mathrm{n}=26)\end{array}$ & $\begin{array}{c}\text { Uberleitungs- } \\
\text { fachkräfte } \\
(\mathrm{n}=21)\end{array}$ & $\begin{array}{c}\text { Mitarbeiter } \\
\text { Hospizverein } \\
(\mathrm{n}=20)\end{array}$ \\
\hline $\mathbf{1 0 - 2 4} \boldsymbol{2 5 - 4 9 €}$ & 5 & - & 1 & 2 \\
\hline $\mathbf{5 0 - 9 9 €}$ & 23 & 1 & 1 & 2 \\
\hline $\mathbf{1 0 0 - 1 4 9} €$ & 45 & 2 & - & 3 \\
\hline $\mathbf{1 5 0}-\mathbf{1 9 9} €$ & 12 & 1 & - & - \\
\hline $\mathbf{2 0 0} €$ & 2 & - & - & 1 \\
\hline
\end{tabular}

Tabelle 13: angemessene Höhe der Vergütung

\subsection{Zusammenfassung der Ergebnisse}

Die vorliegenden Ergebnisse zeigen die Unterschiede in den Beratungen zwischen den, aber auch innerhalb der Gruppen deutlich. Die Unterschiede finden sich in allen durch den Fragebogen erfassten Bereichen.

Klare Beratungsprofile der einzelnen Beratergruppen ließen sich aufgrund der Heterogenität der Antworten innerhalb der Gruppen nicht erstellen. Es konnten dennoch Schwerpunkte bei den Häufigkeiten innerhalb der Gruppen ermittelt werden. Daher erfasst die folgende Schilderung der verschiedenen Beratungen das Bild, wie es von mindestens $50 \%$ der jeweiligen Berater als häufig und/oder als (fast) immer benannt wurde. Besondere Auffälligkeiten sind gesondert benannt.

Unter dieser Prämisse ergibt sich folgendes, zusammenfassendes Bild für die verschiedenen Beratergruppen: 


\subsection{1 Ärzte}

Die teilnehmenden Ärzte stellten mit 10 Beratungen pro Berater (Median) die Beratergruppe mit den wenigsten Beratungen im Jahr 2012. Ihre Kenntnisse für die Beratung hatten sie überwiegend durch Selbststudium und nur zu einem geringen Teil (24\%) durch Schulungen erworben. Damit bildeten sie auch die Gruppe mit den wenigsten Weiterbildungen in diesem Bereich.

Hauptveranlasser der ärztlichen Beratungen waren 61-80 jährige, gesunde Ratsuchende (h/i 87\%). Die Ärzte initiierten das Gespräch nur zu 43\% häufig oder immer selbst. Eine Beratung umfasste 2 Gespräche (h/i 60\%), dauerte insgesamt meistens bis zu 30 Minuten (54\%). Sie erfolgte bei $74 \%$ der Ärzte für den Ratsuchenden unentgeltlich. Selbst die 32\% der Ärzte, die eine Vergütung erhielten, betrachteten die Vergütungssituation als unangemessen. Beraten wurde anhand von Formularen, wobei überwiegend das Formular des Bayerischen Staatsministeriums der Justiz (59\%), daneben aber auch vom Ratsuchenden mitgebrachte, dem Arzt unbekannte Formulare verwendet wurden. Nur die Hälfte der Ärzte empfand die Einbeziehung des zukünftigen Vertreters als hilfreich (53\%) und wies auf die Möglichkeit der Einbeziehung dieser Person in die Beratung hin $(53 \% \mathrm{~h} / \mathrm{i})$. Der zukünftige Vertreter nahm nur bei weniger als einem Fünftel der Ärzte häufig oder fast immer an der Beratung teil (18\%). Damit weist die Teilnahme des Vertreters bei einer ärztlichen Beratung die niedrigste Quote aller Gruppen auf. Etwa jede zweite Beratung schloss mit einer fertigen Patientenverfügung ab (51\%), womit die höchste Quote innerhalb aller befragten Gruppen erreicht wurde. Eine Einbeziehung in die Entscheidungsfindung bei Nichteinwilligungsfähigkeit der Beratenen erfolgte bei $68 \%$ der befragten Ärzte.

Inhaltlich enthielten die Beratungen Hinweise zur Aufbewahrung der Dokumente, die Themen Flüssigkeit und Ernährung am Lebensende, unheilbare, tödlich verlaufende Krankheit, irreversible Bewusstlosigkeit und akuter medizinischer Notfall (alle $\mathrm{h} / \mathrm{i}>\mathbf{8 6 \%} \%$ ). Weniger Bedeutung hatte die Thematisierung der Fragen um psychiatrische Erkrankungen (h/i 55\%) und die Organspende (h/i 44\%). Die weiteren Vorsorgemöglichkeiten Vorsorgevollmacht und Betreuungsverfügung wurden angesprochen (h/i 
$>91 \%$ ). Weniger als die Hälfte der Ärzte gab einen Hinweis auf die Möglichkeit eines Notfallplanes (h/i 44\%) und nur $24 \%$ (h/i) erstellten Notfallpläne.

Weniger als die Hälfte der Ärzte verwies an eine andere Berufsgruppe zur ergänzenden Beratung ( $h / i$ 43\%), so dass nur wenige Hinweise für eine Vernetzung der Beratung zu erkennen sind. Hauptsächlich wurde der Verweis an einen Notar benannt (h/i 49\%).

Die Wünsche der Ärzte richteten sich primär auf eine vermehrte Zusammenarbeit mit Betreuern (43\%), inhaltliche Weiterbildung zu rechtlichen Fragen (59\%) und eine verbesserte Zusammenarbeit im Einzelfall (46\%). $79 \%$ der Ärzte hielten ein routinemäßiges Angebot einer Beratung zur Patientenverfügung ab einem bestimmten Alter für sinnvoll.

\subsubsection{Notare}

Die Notare hatten im Jahr 2012 je 50 Einzelberatungen (Median) durchgeführt und damit die größte Beratungserfahrung im Vergleich aller Gruppen. Die Kenntnisse für die Beratung erwarben sie primär durch Selbststudium $(62 \%)$, aber vielfach auch durch zusätzliche Schulungen oder Weiterbildungen (58\%).

Hauptveranlasser der Beratung waren auch hier gesunde, 61-80jährige Menschen (69\%), aber bei 39\% der Notare auch sie selbst häufig oder immer. Die Notare stellten weitaus die größte Gruppe, die mit Menschen, die erst 41-60 Jahre alt waren, ein Beratungsgespräch führte $(31 \% \mathrm{~h} / \mathrm{i})$. Die für die meisten Ratsuchenden entgeltliche Beratung (69\%) umfasste überwiegend ein Gespräch (h/i 54\%), bei einer ähnlich großen Gruppe auch zwei Gespräche (h/i 46\%). Sie dauerte überwiegend bis zu 30 Minuten (h/i 54\%) und wurde entgeltlich (69\%) anhand von durch den Notar erstellten Formularen geführt (81\%). Ein Hinweis auf die Möglichkeit der Einbeziehung des zukünftigen Vertreters erfolgte bei $66 \%$ der Notare häufig oder fast immer. Eine Teilnahme dieser Personen an dem Gespräch gaben über der Hälfte der Notare an (h/i 62\%). Die meisten Notare bewerteten eine derartige Einbeziehung als hilfreich (65\%). Die Beratungen endeten bei $39 \%$ (h/i) der Notare mit der Fertigstellung der Patientenverfü- 
gung. Die Notare wurden nicht in die Entscheidungsfindung bei Nichteinwilligungsfähigkeit des Patienten einbezogen (0\%).

Inhaltlich bearbeitete diese Berufsgruppe die Themen Flüssigkeit und Ernährung, unheilbare, tödliche Krankheit, irreversible Bewusstlosigkeit, Vorsorgevollmacht, Betreuungsverfügung und gab Hinweise für die Aufbewahrung der Patientenverfügung (alle h/i > 69\%). Auf den akuten medizinischen Notfall (h/i 54\%) und die Möglichkeit der Organspende ging nur knapp die Hälfte (h/i47\%), auf psychiatrische Erkrankungen nur ein Viertel der Notare häufig oder fast immer ein (h/i 24\%). Die Möglichkeit eines Notfallplanes erwähnten nur wenige Notare (h/i 12\%). Sie waren auch nicht an der Erstellung beteiligt.

Die Hälfte der Notare verwies an andere Berufsgruppen zur weiteren Beratung, wobei der Arzt der Experte der Wahl war (89\%).

Die Wünsche der Notare richteten sich hauptsächlich auf ein Feedback (62\%) durch Ärzte (73\%) aus der Anwendungspraxis der Patientenverfügung im konkreten Fall. Ein routinemäßiges Angebot einer Beratung hielt nur knapp die Hälfte dieser Berater für sinnvoll (46\%).

\subsection{3 Überleitungsfachkräfte}

Die Überleitungsfachkräfte führten in 2012 je 25 Beratungsgespräche (Median). Die für ihre Beratungstätigkeit benötigten Kenntnisse eigneten sie sich überwiegend durch Schulungen/Weiterbildungen an (71\%).

Als einzige Gruppe gaben die Überleitungskräfte die Angehörigen als Hauptinitiatoren der Beratung an (62\%). Sie berieten überwiegend unentgeltlich $(76 \%)$ und meistens Menschen, die älter als 81 Jahre waren (57\%). Diese Menschen waren überwiegend schwer erkrankt (h/i 62\%) oder hatten nur noch eine Lebenswartung von weniger als 6 Monaten (h/i24\%). Die meisten Beratungen umfassten zwei Gespräche (48\%), dauerten bis zu 30 Minuten (43\%) und wurden anhand des Formulars des Bayerischen Staatsministeriums der Justiz geführt (62\%). Die Überleitungsfachkräfte bildeten die Beratergruppe, die am häufigsten auf die Möglichkeit der Einbeziehung des zukünftigen Vertreters hinwies (h/i $86 \%$ ), und dieser auch weitaus am häufigsten teilnahm (h/i 85\%). Eine 
Fertigstellung der Patientenverfügung innerhalb der Beratung erfolgte nur bei $29 \%$ der Befragten und stellte damit die niedrigste Quote unter den Beratergruppen dar. Nur ein Viertel der Überleitungsfachkräfte gab an, in die Entscheidungsfindung bei Einwilligungsunfähigkeit der Patienten einbezogen zu werden.

Inhaltlich umfasste die Beratung Hinweise zur Aufbewahrung der Patientenverfügung sowie die Themen Flüssigkeit und Ernährung, unheilbare, tödliche Krankheit, akuter medizinischer Notfall, Vorsorgevollmacht und Betreuungsverfügung (alle $\mathrm{h} / \mathrm{i}>52 \%$ ). Die Themen irreversible Bewusstlosigkeit, psychiatrische Erkrankung und Möglichkeit eines Notfallplanes spielten eine nachgelagerte Rolle ( $\mathrm{h} / \mathrm{i} 43-48 \%$ ). Das Thema Organspende fand kaum Eingang in die Beratung (h/i 15\%). Eine Beteiligung an der Erstellung von Notfallplänen wurde nur von 10\% dieser Berater benannt.

Die Überleitungsfachkräfte verwiesen im Vergleich zu den anderen Gruppen am häufigsten zur ergänzenden Beratung an andere Berufsgruppen (h/i $77 \%)$, vornehmlich Ärzte (76\%).

Die Wünsche der Überleitungsfachkräfte richteten sich hauptsächlich auf eine verstärkte Zusammenarbeit mit dem Arzt (62\%) im Einzelfall (52\%) und auf die Erweiterung der Kenntnisse im rechtlichen Bereich (52\%). Ein routinemäßiges Angebot einer Beratung hielt gut die Hälfte dieser Berater für sinnvoll (57\%).

\subsubsection{Mitarbeiter der Hospizvereine}

Die Mitarbeiter der Hospizvereine führten in 2012 je 35 Beratungsgespräche (Median). Das für die Beratung benötigte Wissen erwarben sie sich überwiegend $(75 \%)$ durch Schulungen/Weiterbildungen.

Hauptveranlasser der Beratung waren die Ratsuchenden selbst (h/i 80\%), die überwiegend bereits schwer erkrankt ( $\mathrm{h} / \mathrm{i}$ 65\%) und zwischen 61-80 Jahre alt waren (90\%). Die unentgeltliche Beratung (90\%) dauerte bei der Hälfte der Berater 60-90 Minuten. Die Mitarbeiter der Hospizvereine verwendeten damit im Vergleich mit den anderen Gruppen am meisten Zeit für eine Beratung. Sie umfasste zwei Gespräche (h/i 60\%) und basierte bei $85 \%$ der Berater auf dem Formular des Bayerischen Ministeriums der Justiz. Damit fand dieses Formular seine häufigste Verwendung bei den 
Mitarbeitern der Hospizvereine. Diese Berater verwiesen sehr häufig auf die Möglichkeit der Teilnahme des zukünftigen Vertreters an der Beratung (h/i 85\%), allerdings findet sie nur in gut der Hälfte aller Beratungen häufig oder fast immer statt (h/l 55\%). Die Quote der innerhalb der Beratung fertiggestellten Patientenverfügungen lag mit $45 \%$ nach den Hausärzten am zweithöchsten. Zu einer Einbeziehung in die Entscheidungsfindung bei Nichteinwilligungsfähigkeit des Patienten kam es bei $60 \%$ dieser Berater. Inhaltlich wurden die Themen Flüssigkeit und Ernährung, unheilbare tödlich verlaufende Krankheit, irreversible Bewusstlosigkeit, akuter medizinischer Notfall, Vorsorgevollmacht, Betreuungsverfügung und Möglichkeit eines Notfallplanes von der Beratung erfasst (alle $\mathrm{h} / \mathrm{i}>55 \%$ ). Weniger Berücksichtigung fanden die Fragen um psychiatrische Erkrankungen (h/i $25 \%$ ) und Organspende (h/i 30\%). Eine Beteiligung bei der Erstellung von Notfallplänen berichteten 45\% dieser Berater.

Ein Verweis an andere Berufsgruppen erfolgte eher weniger häufig (h/i $40 \%)$ und dann primär an einen Arzt (65\%).

Die Wünsche der Mitarbeiter der Hospizvereine richteten sich primär auf eine verstärkte Zusammenarbeit im Einzelfall (65\%) mit dem Arzt (80\%) und auf eine Erweiterung medizinischer Kenntnisse (45\%). Ein routinemäBiges Beratungsangebot schätzten 75\% der Berater als sinnvoll ein. 


\section{Diskussion}

Die vorliegende Studie untersucht erstmals in Deutschland die Beratung zur Patientenverfügung durch verschiedene Beratergruppen einer bestimmten Region. Sie ermöglicht neben einem Einblick in die Struktur und den Inhalt der Beratung, auch Erkenntnisse über die Wünsche der Berater und Möglichkeiten der Weiterentwicklung der Beratung.

Die Einordnung der Ergebnisse der Studie bezieht, neben der bestehenden Literatur auch die best practice umfassender, international etablierter ACP Programme ein. Derartige ACP Programme beinhalten ein primär weniger auf das Erstellen von Dokumenten, sondern umfassender auf die Gestaltung eines professionell begleiteten, kommunikativen Prozesses zwischen dem ratsuchenden Menschen, seinen Behandelnden und Angehörigen ausgerichtetes Konzept (Wilkinson 2011). Ziel ist es, den Menschen nicht nur bei der Vorsorge für den Fall der Einwilligungsunfähigkeit zu unterstützen, sondern inn auch im Prozess der Auseinandersetzung mit dem (eventuell schon nahenden) Lebensende zu begleiten.

Umfassende ACP Programme beruhen auf zwei Säulen: Die erste Säule bildet ein professionell begleiteter Gesprächsprozess (facilitation), die zweite die regionale Implementierung von Standards und Routinen, die die Kenntnis und Umsetzung der Wünsche des Patienten/Bewohners gewährleisten sollen.

Nachhaltigen Einfluss auf die Entwicklung von ACP Programmen haben insbesondere das seit 1991 in La Crosse (Wisconsin) entwickelte Programm „Respecting Choices“ (Gunderson), die Forschergruppe um William Silvester und Karen Detering in Australien (Detering et al. 2010), sowie in Deutschland das durch Jürgen in der Schmitten und Georg Marckmann durchgeführte Projekt „Beizeiten Begleiten” (In der Schmitten et al. 2014). Wie die Reviews von Brinkmann-Stoppelenburg und Houben belegen, kann eine derartige systematische, patientenzentrierte und vernetzte Vorgehensweise nachweislich zu einer Verbesserung der Versorgung der Menschen insbesondere am Lebensende beitragen (BrinkmanStoppelenburg et al. 2014; Houben et al. 2014). So führt sie beispielweise zu einem verbesserten Wissen um die Wünsche des Patienten (Kirchhoff 
et al. 2012), einer höheren Zahl an Patientenverfügungen (Hammes et al. 2010; In der Schmitten et al. 2014), einer besseren Auffindbarkeit und Klarheit der Vorsorgedokumente (Hammes et al. 2010; Hammes et al. 2012), einer größeren Übereinstimmung der dokumentierten Wünsche mit der tatsächlich am Lebensende erfolgten Behandlung (Detering et al. 2010; Hammes et al. 2010) und der Vermeidung von nicht gewünschten Krankenhausaufenthalten (Fromme et al. 2014; Gozalo et al. 2011). Auch die Zufriedenheit von Patienten und Angehörigen mit der Behandlung in der letzten Lebensphase steigt (Detering et al. 2010). Die Angehörigen leiden weniger unter Depressionen, post-traumatischem Stress und Angst (Detering et al. 2010; Hammes et al. 2012).

Daher soll die best practice der Gesprächsprozesse von ACP Programmen in die Diskussion einbezogen werden.

\subsection{Struktur der Beratung}

Im Hinblick auf Struktur und Inhalt der Beratung ist bemerkenswert, dass sowohl zwischen den Beratergruppen als auch innerhalb der Gruppen unterschiedliche Vorgehensweisen und Inhalte der Beratung erkennbar sind. Die Unterschiede finden sich in allen durch den Fragebogen erfassten Bereichen.

Eine Beschreibung klarer Beratungsprofile für die einzelnen Gruppen war aufgrund der Heterogenität der Antworten der Teilnehmer nicht möglich. Es konnten jedoch Hinweise auf Schwerpunkte und Besonderheiten der Beratung bei den einzelnen Gruppen herausgearbeitet werden.

\subsubsection{Initiierung der Beratung}

Die Initiierung der Beratung erfolgte bei allen Gruppen, außer bei den Überleitungsfachkräften, hauptsächlich durch den Ratsuchenden, gefolgt von den Beratern und den Angehörigen.

Die hohe Quote der Veranlassung einer ärztlichen Beratung durch den Ratsuchenden selbst ( $\mathrm{h} / \mathrm{i}$ 87\%) deckt sich mit der Haltung vieler Hausärzte, der Patient solle die Beratung einfordern (De Vleminck et al. 2013; De Vleminck et al. 2014). Auch die Empfehlungen der Bundesärztekammer 
raten, in der Regel dem Patienten die Eröffnung des Gesprächs zur Patientenverfügung zu überlassen (Bundesärztekammer 2013).

Dagegen gehört ein proaktives Angebot der Beratung bei umfassenden ACP Programme zur best practice (Hammes et al. 2010; Silvester et al. 2013a; Wilkinson 2011). Verschiedene Autoren fordern ebenfalls eine stärkere Initiative der Hausärzte (Hubert et al. 2013; Nauck et al. 2014). Diese Forderung unterstreichen $79 \%$ der ärztlichen Teilnehmer der vorliegenden Studie, die sogar ein routinemäßiges Angebot einer Beratung ab einem bestimmten Alter für sinnvoll hielten. Dennoch gaben weniger als die Hälfte der befragten Hausärzte (43\%) an, häufig oder fast immer selbst die Initiative zur Beratung zu ergreifen. Eine ähnliche Zurückhaltung von Ärzten beschrieben Studien für den klinischen Bereich (Hubert et al. 2013; Knauss 2013). Dort wurden nur $10 \%$ bzw. 35\% der befragten Patienten auf das Thema Vorsorge angesprochen. Eine andere Studie weist auf die Ablehnung einer Beratung durch Ärzte hin, selbst wenn sie von Patienten danach gefragt werden (Nauck et al. 2014). Fraglich ist, welchen Hintergrund diese Diskrepanz zwischen der tatsächlichen und der für sinnvoll gehalten Häufigkeit der Initiierung der Beratung durch Hausärzte hat. Die Gründe, warum die Beratung nicht häufiger aktiv angeboten wurde, sind in der vorliegenden Studie nicht erfragt worden. Untersuchungen zur Identifikation der Barrieren weisen beispielsweise auf Unsicherheiten bei der Gesprächsführung zum Thema Sterben, im rechtlichen Bereich oder der Frage nach dem richtigen Zeitpunkt für ein Gespräch (Burchardi et al. 2005). Hinzu kommt die Angst, dem Patienten die Hoffnung zu nehmen und die Sorge vor einem hohen Zeitbedarf für die Gespräche (Almack et al. 2012; Barnes et al. 2011; Morrison et al. 1994; van der Steen et al. 2014). Möglicherweise fehlte etlichen Ärzten das Wissen um die positiven Effekte der Thematisierung der Vorsorge: So beschreiben Patienten Gefühle der Erleichterung und der Wahrnehmung von Fürsorge, ebenso wie die Reduktion von Angst und Stress (Detering et al. 2010; Knauss 2013). Auch die Zufriedenheit der Patienten mit dem Hausarzt kann bei der Initiative durch diesen steigen, wie es die Studie von Tierney mit älteren, chronisch kranken Menschen belegt (Tierney et al. 2001). 
Fraglich ist, ob durch die auch aus anderen Ländern bekannte Zurückhaltung der Ärzte (Barnes et al. 2011; Sahm et al. 2005b; Torjesen 2014) nicht Menschen ohne Beratung bleiben, die sie eigentlich in Anspruch nehmen möchten. Es gibt Hinweise darauf, dass viele Menschen auf die Frage des Arztes, ob eine Beratung gewünscht ist, warten (Alano et al. 2010; Almack et al. 2012; Hubert et al. 2013; Jackson et al. 2009; Knauss 2013; Sahm et al. 2005b; Sharp et al. 2013). Etliche Patienten gehen davon aus, der Hausarzt habe, obwohl er vielfach ihr bevorzugter Ansprechpartner wäre (Hubert et al. 2013), keine Zeit für eine Beratung (Schickedanz et al. 2009).

Dessen sind sich Ärzte, gerade wenn sie wie die Teilnehmer dieser Studie, überwiegend gesunde Menschen beraten, möglicherweise nicht immer bewusst (Almack et al. 2012; De Vleminck et al. 2013).

Versorgende Einrichtungen wie Altersheime spielten kaum eine Rolle als Veranlasser einer ärztlichen Beratung zur Patientenverfügung (h/i 4\%). Dieses Ergebnis gibt Hinweise darauf, dass es keine feste Zusammenarbeit zwischen den Heimen und den dort tätigen niedergelassenen Ärzten zum Thema Vorsorge gibt. Von einem Bedarf an Beratung ist in diesem Setting jedoch auszugehen: Wie bereits festgestellt, korrelieren steigendes Alter und das Interesse an Vorsorge (Forschungsgruppe Wahlen 2012). Diesen Zusammenhang bestätigen auch die Aussagen der Überleitungsfachkräfte, die vornehmlich über 80jährige Menschen in den Altersheimen beraten. Fraglich ist daher, ob und in welchem Umfang Ärzte in diesen Einrichtungen beraten und wie ihre Rolle neben den Überleitungsfachkräften ist. Daneben ist unklar, wer die Einwilligungsfähigkeit der Bewohner, die häufig aufgrund von Demenzen oder anderen gesundheitlichen Veränderungen schwierig zu ermitteln ist, beurteilt. Die ärztliche Beurteilung und Dokumentation der Einwilligungsfähigkeit wäre hilfreich um die Validität einer Patientenverfügung einschätzen zu können. Eine derartige Validierung der Vorsorgewünsche ist fester Bestandteil von ACP Programmen und gehört zu deren best practice (In der Schmitten et al. 2014; Silvester et al. 2013b). 
Bei den Notaren waren die Ratsuchenden ebenfalls die Hauptveranlasser der Beratung (h/i 100\%). Die Motivation von Menschen, notarielle Beratung aktiv zu suchen, wurde bereits von Becker et. al beschrieben (Becker et al. 2010). Sie beruht danach vielfach auf dem hohen Vertrauen, das notariell verfassten Dokumenten entgegengebracht wird. Dieses Vertrauen scheint den Bedarf an detaillierten medizinischen Informationen zu überwiegen (Becker et al. 2010). Bemerkenswert ist bei den Notaren der mit 39\% vergleichsweise hohe Anteil der Notare, die selbst häufig oder immer die Beratung veranlassten. Notare erhalten außerhalb von Vorträgen o.ä. nur Kontakt zu Ratsuchenden, wenn diese den Notar zu einer Beratung aufsuchen. Man kann daher davon auszugehen, dass viele Notare, z.B. im Rahmen der Beratung zum Testament, auch auf die Möglichkeiten einer Vorsorge für den Fall der Nichteinwilligungsfähigkeit hinweisen. Die Notare spielen daher eine große Rolle bei der Verbreitung des Wissens um die Vorsorgemöglichkeiten und der Initiierung der Beratung, zumal sie die meisten Beratungen pro Berater durchführen ${ }^{10}$.

Die einzige Beratergruppe, bei der nicht die Ratsuchenden selbst, sondern deren Angehörige Hauptveranlasser der Beratung waren ( $\mathrm{h} / \mathrm{i}=63 \%$ ), ist die der Überleitungsfachkräfte. Dieses Ergebnis zeigt deutlich, welche Bedeutung das Vorhandensein von Angehörigen, die sich um die Bewohner kümmern, für die Initiierung einer Vorausplanung heute hat. Viele Bewohner der Altenheime sind in ihrer Selbstständigkeit eingeschränkt und daher auf die Unterstützung anderer Menschen, vor allem ihrer Angehörigen angewiesen. Das Bedürfnis der Bewohner nach Vorsorge ist vielfach vorhanden (Sharp et al. 2013). Das vorliegende Ergebnis kann daher als Hinweis für die Institutionen gewertet werden, insbesondere für die steigende Zahl von Menschen, die keine Angehörigen mehr haben, aktiv Angebote zur Vorausplanung zu machen. 48\% der befragten Überleitungsfachkräfte initiieren bereits heute häufig oder fast immer die Beratung. Dennoch besteht noch ein erheblicher Bedarf: So haben in der durch den Deutschen Hospiz- und Palliativverband (DHPV) in Auftrag gegebenen

\footnotetext{
${ }^{10}$ Die notarielle Beratung wird vielfach gesucht, obwohl sie überwiegend entgeltlich erfolgt (Abb. 31).
} 
repräsentativen Studie der Forschungsgruppe Wahlen Telefonfeld (Forschungsgruppe Wahlen 2012) 42\% aller Deutschen, die 60 Jahre alt und älter sind, angegeben, eine Patientenverfügung verfasst zu haben. Die Studie von Sommer in Altenheimen ergab eine Häufigkeit des Vorliegens einer Patientenverfügung mit einer Spanne von 2 - 22\% je nach Einrichtung (In der Schmitten et al. 2014; Sommer et al. 2012). Diese Studie stützt sich nicht auf Selbstauskünfte, sondern hat das tatsächliche Vorliegen von Patientenverfügungen in den Einrichtungen geprüft. Damit bleibt die Zahl der Patientenverfügungen in Altenheimen deutlich hinter der durch Selbstauskünfte ermittelten Prävalenz in der allgemeinen Bevölkerung zurück. Möglicherweise ist die deutlich höhere Prävalenz bei den Selbstauskünften der Befragten durch den Gedanken der „sozialen Erwünschtheit“ beeinflusst. Dennoch wird von einer erheblichen Diskrepanz der Prävalenzen auszugehen sein.

Als weiteren Hinweis, der den Bedarf an der Weiterentwicklung der Beratung in den Heimen unterstreicht, kann man auch die aufgezeigte nachrangige Rolle die versorgende Einrichtungen bei der Initiierung der Beratung sehen (max. 15\% h/i). Die Interventionsstudie von In der Schmitten belegt, welche Einflussmöglichkeiten auf die Verbesserung der Vorausplanung gerade Altenheime haben (In der Schmitten et al. 2014).

Die Beratung durch Mitarbeiter von Hospizvereinen veranlassten bei $80 \%$ der Berater die Ratsuchenden selbst (h/i), nur 30\% der Berater wurden häufig selbst initiativ. Dagegen spielten Angehörige, ebenso wie bei den Überleitungsfachkräften, eine bedeutende Rolle (h/i 45\%). Es gibt Untersuchungen, die belegen, dass das Erleben von Leiden und Versterben naher Angehöriger ein wesentlicher Auslöser für die Verfassung von Patientenverfügungen für die eigene Person ist (Amjad et al. 2014; Carr 2012; Lang und Wagner 2007). Mitarbeiter von Hospizvereinen beraten zu einem großen Teil Menschen, die bereits schwer erkrankt sind (h/i 65\%) oder eine Lebenserwartung von weniger als sechs Monaten haben ( $\mathrm{h} / \mathrm{i}$ 55\%). Möglicherweise ist das akute Erleben des Leidens oder des nahenden Todes eines Familienmitgliedes, wie es in der hospizlichen Versorgung geschieht, auch eine Motivation für die Angehörigen, sich nicht nur 
um die gesundheitliche Vorausplanung für sich selbst, sondern auch des kranken und /oder hochaltrigen Familienmitgliedes zu kümmern und hierfür eine Beratung zu initiieren. Auf der anderen Seite gibt es Hinweise auf die fehlende Bereitschaft Angehöriger, das Thema Vorausplanung aufzugreifen (van der Steen et al. 2014).

Weitere Forschung zur Rolle der Angehörigen bei der gesundheitlichen Vorausplanung wäre daher sinnvoll.

Nicht jeder Mensch möchte Vorsorge durch eine Patientenverfügung treffen (Voltz et al. 1998). Viele Menschen warten jedoch darauf, auf dieses Thema angesprochen zu werden (Hubert et al. 2013). Daher lässt sich zusammenfassend feststellen, dass alle Beratergruppen mehr Initiative zur Beratung ergreifen sollten, um all denen, die aufgrund mangelnder oder fehlerhafter Information nicht um die Bedeutung der Vorsorge wissen, ein Angebot zur Auseinandersetzung mit dem Thema machen zu können.

Umfassende ACP Programme enthalten ein routinemäßiges Angebot der Beratung über die Vorsorgemöglichkeiten ab einem bestimmten Alter (Hammes et al. 2010). Auch die vorliegende Studie zeigt, im Unterschied zu einer von Sahm im Jahr 2005 veröffentlichten Untersuchung (Sahm et al. 2005b), dass große Teile der Berater ein routinemäßiges Angebot für sinnvoll halten (46-79\%). Dies überrascht insbesondere bei den $79 \%$ der Ärzte, die sich dafür aussprachen, obwohl nach den Ergebnissen dieser Studie weniger als die Hälfte der Mitglieder dieser Berufsgruppe häufig oder fast immer die Initiative zur Beratung ergreift (h/i 43\%). Zurückhaltender waren die Notare. Hier sprach sich knapp die Hälfte der Befragten (46\%) für ein derartiges Angebot, die andere Hälfte (50\%) dagegen aus. Möglicherweise hängen die Befürwortung eines routinemäßigen Beratungsangebotes mit der Häufigkeit zusammen, mit der die verschiedenen Berater zur Entscheidungsfindung bei Nichteinwilligungsfähigkeit zugezogen werden, und so die Probleme der Entscheidungsfindung in der Praxis erleben (Abb. 21). Grundsätzlich wäre weitere Forschung zu den Gründen für die unterschiedliche Einstellung zu einem routinemäßigen Beratungsangebot sinnvoll. 
Betrachtet man die Zahlen der Patientenverfügungen, die nach einem strukturierten, regelhaften ACP Angebot vorliegen (Hammes et al. 2010;In der Schmitten et al. 2014) und die in der Literatur beschriebene Bereitschaft der Menschen zur Vorsorge (Burchardi et al. 2005; Sahm et al. 2005a; van Oorschot et al. 2004), so kann man davon ausgehen, dass in Deutschland noch ein erhebliches ungedecktes, individuelles Bedürfnis nach qualitativ hochwertiger Beratung zur gesundheitlichen Vorausplanung besteht.

\subsubsection{Alter der Ratsuchenden}

Das Alter der bei Ärzten, Notaren und Mitarbeitern von Hospizvereinen Ratsuchenden lag überwiegend bei 61-80 Jahren. Diese Altersstruktur deckt sich mit den Untersuchungsergebnissen der durch den Deutschen Hospiz- und Palliativverband in Auftrag gegebenen Studie, bei der die über 60jährigen die größte Prävalenz von Patientenverfügungen (42\%) aufwies (Forschungsgruppe Wahlen 2012). In der Literatur gibt es ebenfalls zahlreiche Hinweise einer Assoziierung von höherem Lebensalter und dem Interesse und der Bereitschaft zur Vorsorge (Alano et al. 2010; Hubert et al. 2013; Knauss 2013; Lang und Wagner 2007). Die Notare bildeten die einzige Gruppe, die in nennenswertem Umfang jüngere Menschen im Alter von 41 - 60 Jahren zum Thema Vorsorgeplanung erreichte (31\%). Dieses Ergebnis lässt auf eine große Bereitschaft zur Vorsorge auch von jüngeren Menschen schließen. Es wäre hilfreich, mehr über die Motivation und die besonderen Bedürfnisse der Menschen dieser Altersklasse zu erfahren, um diese Erkenntnisse für die Weiterentwicklung der Beratungsangebote verwenden zu können.

Bei über der Hälfte der Überleitungsfachkräfte formten Menschen, die älter als 81 Jahre waren, die größte Gruppe der Beratenen (57\%). Die Überleitungsfachkräfte stellten somit die einzigen Berater, bei denen die über 80jährigen die größte Gruppe der Ratsuchenden bildete. Menschen mit einer Lebenserwartung von unter sechs Monaten stellten die größte Gruppe der durch Mitarbeiter von Hospizvereinen beratenen dar (h/i 55\%). Diese Aussagen korrespondieren mit den Arbeitsumfeldern der Befragten. 


\subsubsection{Gesundheitszustand der Ratsuchenden}

Den Gesundheitszustand der überwiegenden Zahl der bei innen Ratsuchenden bezeichneten die Hausärzte (h/i 77\%) und Notare (h/i 100\%) als nach ihrer Kenntnis "gesund“. Damit zeigt sich, dass viele Menschen heute bereits dann Vorsorge treffen wollen, wenn Sie noch nicht schwer erkrankt sind. Dieses Forschungsergebnis unterstreicht die in der Literatur und ACP Programmen vertretene Forderung nach einem frühzeitigen Beratungsangebot (Nauck et al. 2014).

Bei den Hausärzten war der Anteil der Menschen, die bereits schwer erkrankt waren oder eine Lebenserwartung von unter sechs Monaten hatten, an der Beratung eher klein (schwer erkrankt $\mathrm{h}=28 \%,<6$ Monate $\mathrm{h} / \mathrm{i}=8 \%$ ). Diese Aussage steht in einem Spannungsverhältnis zu dem in der Literatur beschriebenen, steigenden Bedürfnis nach Vorsorge im Fall eigener schwerer Erkrankung (Sahm et al. 2005a; van Wijmen et al. 2014). Auch die Bundesärztekammer weist in ihren Empfehlungen darauf hin, dass es die Fürsorgepflicht des Arztes bei schwerer Erkrankung gebieten kann, eine Beratung anzuregen (Bundesärztekammer 2013). Die best practice von ACP Programmen beschreibt derartige Situationen als Auslöser für die Initiierung der Beratung („Wären Sie erstaunt, wenn ihr Patient im nächsten Jahr versterben würde?") (Clayton 2011). Fraglich ist daher, wieso die Beratung schwerkranker Menschen bei den Ärzten keine größere Rolle spielte, obwohl der Hausarzt vielfach der bevorzugte und fachlich geeignete Gesprächspartner wäre (Hubert et al. 2013). Die Gründe hierfür können sowohl in der Sphäre der kranken Menschen (Nauck et al. 2014), als auch in der Sphäre der Ärzte liegen. Auf die in der Literatur beschriebenen Gesprächsbarrieren wurde bereits hingewiesen ( s. 4.1.1).

Notare berieten kaum Menschen, die bereits schwer erkrankt waren $(\mathrm{h}=4 \%)$ oder eine Lebenserwartung von unter sechs Monaten hatten $(0 \%)$. Dieses Ergebnis könnte seine Ursache entweder darin haben, dass schwer erkrankte Menschen von vorneherein eher die Beratung der anderen, medizinnahen Berufsgruppen aufsuchen. Eine weitere Erklärung wäre 
ein möglicherweise bestehendes Bewusstsein der Notare für ihre ${ }^{11}$ begrenzten medizinischen Kenntnisse, die insbesondere im Fall einer schweren Erkrankung des Ratsuchenden Voraussetzung für eine tragfähige Vorausplanung sind. Es wäre möglich, dass sie daher im Fall schwerer Erkrankung der Ratsuchenden direkt an die Beratung durch den Arzt verweisen (Abb.24).

Überleitungsfachkräfte und Mitarbeiter von Hospizvereinen führten ihre Beratungsgespräche meistens mit bereits schwerkranken Menschen ( $h / i$ $62 \%, 63 \%$ ) und vielfach mit denjenigen, die nur noch eine kurze Lebenserwartung hatten (h/i 24\%, 55\%).

Zwischen 5 - 16\% der nichtärztlichen Berater kannten den Gesundheitszustand der Ratsuchenden nicht. Zu klären wäre hier, welche Art der Beratung von diesen Beratern geleistet wurde. Eine tragfähige Patientenverfügung kann nur unter Einbeziehung des Gesundheitszustandes ${ }^{12}$ und der daraus resultierenden Anforderungen an die Vorsorge verfasst werden (May et al. 2005; Silvester et al. 2013a). Auch bei ACP Programmen ist die Kenntnis des Gesundheitszustandes des Ratsuchenden Basis der Gespräche über die Vorausplanung (Silvester et al. 2013b). Möglicherweise beschränkte sich die Tätigkeit der genannten Berater auf eine umfassende Information zu den Vorsorgemöglichkeiten und stellte keine individualisierte Beratung im engeren Sinne dar.

\subsubsection{Dauer und Anzahl der Gespräche}

Das Ergebnis der vorliegenden Untersuchung zu Dauer und Anzahl der Gespräche im Rahmen einer Beratung lässt fraglich erscheinen, ob die Beratung bereits den vielfach geforderten Prozesscharakter hat (Lang und Wagner 2007; May et al. 2005; Tamayo-Velázquez et al. 2010; van Oorschot et al. 2012) und den nötigen Raum zur Information und Reflexion gibt: So benötigten die Gruppen überwiegend 1-2 Gespräche für eine Be-

\footnotetext{
11 in der Regel

${ }^{12}$ Es sollte zumindest bekannt sein, ob bereits Krankheitsbilder bestehen, um diese gegebenfalls besprechen und den Umgang mit innen in die Vorausplanung einbeziehen zu können.
} 
ratung und etwa die Hälfte der Berater schätze die gesamte Beratungsdauer auf maximal 30 Minuten (ein weiteres Drittel auf 30-60 Minuten). Zur best practice von ACP Programmen gehört eine umfassende Information und eine tiefgehende Diskussion (Silvester et al. 2013a). Die Beratungsgespräche werden dabei auf mehrere Termine verteilt (In der Schmitten et al. 2014b). Bei bisherigen ACP Programmen wurden zwischen $60-100$ Minuten (Median) für die Gesprächsbegleitung aufgewendet (Detering et al. 2010; In der Schmitten et al. 2014). Diesen Zeitaufwand gaben nur $50 \%$ der Hospizmitarbeiter an. Über die Gründe, warum häufig nur ein relativ kurzes Gespräch geführt wird, und die Frage, ob sie dem Bereich der Berater oder der Ratsuchenden zuzuordnen sind, wäre weitere Forschung wünschenswert.

\subsubsection{Verwendete Formulare}

Grundlage der Beratung bildeten nach Aussage der überwiegenden Anzahl der Teilnehmer der Studie verschiedene Formulare. Dieses Ergebnis deckt sich mit der Studie von Nauck, die ebenfalls die häufige Verwendung von standardisierten Formularen beschreibt (Nauck et al. 2014). Am häufigsten wurde in der vorliegenden Studie das Formular des Bayerischen Staatsministeriums der Justiz genannt. Über die Hälfte der NichtNotare (59-85\%) nutzten dieses Formular. Selbst 31\% der Notare gaben an, es zu verwenden. Es hat damit einen hohen Bekanntheitsgrad und ist von herausragender Bedeutung für die gesundheitliche Vorausplanung in der Region, auch wenn in der Studie keine Aussagen über die Häufigkeit der Verwendung in den Beratungen durch den einzelnen Berater erhoben wurden.

$81 \%$ der Notare, $21 \%$ der Hausärzte und $15 \%$ der Hospizmitarbeiter verwendeten daneben selbst entwickelte Formulare. Hier ist zu hinterfragen, welchen Inhalt und welche Qualität diese Formulare haben.

Die gleiche Frage stellt sich, wenn $67-92 \%$ der Berater auch anhand von Formularen berieten, die der Ratsuchende selbst mitgebracht hat, die vom Berater aber in der Regel nicht verwendet werden. Es wäre zu untersuchen, ob die z.B. in den Standards für die Beratung zu Patientenverfügun- 
gen (May et al. 2005) geforderten Fähigkeiten zur Prüfung der Qualität solcher Formulare bei allen Berufsgruppen vorhanden sind.

\subsubsection{Vorsorgevollmacht und Betreuungsverfügung}

Über die Bedeutung der Vorsorgeinstrumente Vorsorgevollmacht und Betreuungsverfügung informierten die Berater zwar sehr häufig, aber noch nicht (fast) immer (i 66-92\%). Diese Information wird sowohl von der Bundesärztekammer, den Standards für die Beratung zur Patientenverfügung, als auch von den ACP Programmen als wesentlicher Bestandteil der Vorsorgeplanung gesehen (Bundesärztekammer 2013; May et al. 2005; Silvester et al. 2013b). Auf diesem Wege soll die rechtzeitige Ermittlung und Legitimierung eines zukünftigen Vertreters gefördert werden. Der Studie des DHPV zufolge gehen, je nach Alter, zwischen 66 und 90\% der Bevölkerung davon aus, dass Angehörige, Freunde oder Nachbarn sich im Fall schwerer Krankheit um sie kümmern werden (Forschungsgruppe Wahlen 2012). Viele Menschen verfügen jedoch über keine ausreichenden Kenntnisse über das Erfordernis der Legitimation eines Vertreters durch Vorsorgevollmacht oder Bestellung als Betreuer (Sahm et al. 2005a; SchulteStrathaus 2014; Wilke et al. 2014). Daneben bestehen vielfach Fehlvorstellungen über die Vertretungsmacht Angehöriger (Schöffner et al. 2012; van Oorschot et al. 2012). Daher ist eine konsequente und frühzeitige Information zu Bedeutung und Gestaltung der Vertretung umso bedeutsamer.

\subsubsection{Einbeziehung des Vertreters der Ratsuchenden}

Die Einbeziehung des zukünftigen Vertreters des Ratsuchenden in das Beratungsgespräch handhabten die Berater sehr unterschiedlich. Nur zwischen 53-86 \% der Berater wiesen häufig oder fast immer auf diese Möglichkeit hin. Die Teilnahme der zukünftigen Vertreter an der Beratung erfolgte, abhängig von der Beratergruppe, bei 18-85\% der Befragten häufig oder immer, also sehr uneinheitlich.

Die Ärzte zeigten hierbei die größte Zurückhaltung (Hinweis h/i 53\%). Nur $18 \%$ von ihnen berichteten über eine häufige Teilnahme der zukünftigen Vertreter an der Beratung. Entgegen dem in den Empfehlungen der Bun- 
desärztekammer (Bundesärztekammer 2013) geäußerten Hinweis auf den hilfreichen Charakter einer Einbeziehung, stellten Ärzte die einzige Beratergruppe dar, bei der nur etwa die Hälfte der Mitglieder die Einbeziehung als hilfreich empfand, und sogar $10 \%$ als störend. Alle anderen Beratergruppen schilderten die Einbeziehung zu mindesten zwei Dritteln als hilfreich (65-95\%) und die zukünftigen Vertreter als häufige Teilnehmer der Beratung (h/i 55-85\%). Das Verhalten der Ärzte mag seine Ursache in der Arzt-Patienten-Beziehung haben oder auch in dem Wunsch mancher Patienten, zwar über den Tod zu sprechen, jedoch nicht mit Familienmitgliedern (Collins und Lehane 2013). Auch manche Angehörige wollen sich nicht mit dieser Thematik auseinandersetzen (Sharp et al. 2013; van der Steen et al. 2014). Dennoch möchten die meisten Menschen ihre Angehörigen einbeziehen und wünschen sich, dass Entscheidungen gemeinsam von ihnen, den Angehörigen und dem Arzt getroffen werden. So werden Angehörige häufig als Vertreter eingesetzt (Torke et al. 2014; Voltz et al. 1998). Aufgabe des Vertreters ist es, die Wünsche des Patienten zu ermitteln und umzusetzen ( $\$ 1901$ III, 1901a I BGB). Dass es sich hierbei um eine komplexe Aufgabe handelt, zeigt sich unter anderem daran, dass ungefähr ein Drittel der Vertreter den Willen des Patienten aus unterschiedlichen Gründen nicht kennt oder nicht umsetzt (Fagerlin et al. 2001; Hauke et al. 2011; Inoue und Moorman 2014; Kuehlmeyer et al. 2012; Shalowitz et al. 2006; Vig et al. 2011). Wie die Studie von Nauck belegt, sind viele Vertreter nicht einmal über ihre Bevollmächtigung informiert und haben auch keine Kenntnisse über die Wünsche des zu Vertretenden (Nauck et al. 2014). Damit ist die Ermittlung und Umsetzung der Patientenwillens und somit die Wahrung der Selbstbestimmung des Patienten erheblich erschwert. Viele Menschen wollen Vorsorge treffen, um Ihre Angehörigen zu entlasten (van Oorschot et al. 2012; van Wijmen et al. 2014). Fehlt es jedoch an ausreichender Kommunikation, wird dieses Ziel nicht erreicht: mangelhafte oder fehlende Kommunikation über die Wünsche des zu Vertretenden erhöht die ohnehin schon hohe Belastung der Stellvertreter nochmals (Wendler und Rid 2011). Daher liegt eine vordringliche Aufgabe der Berater darin, dem Ratsuchenden und, wenn möglich, dem zukünftigen Stellvertreter diese Zusammenhänge zu erklären und den zu- 
künftigen Vertreter möglichst in die Beratung einzubeziehen, wie es auch die Bundesärztekammer und ACP Programme als best practice empfehlen (Bundesärztekammer 2013; Silvester et al. 2013a; Torke et al. 2014). Diesem Anspruch genügt die derzeitige Beratungssituation noch nicht zuverlässig.

\subsubsection{Ausbildung der Berater für die Beratung}

Die Gründe für die unterschiedlichen Erfahrungen mit der Einbeziehung der zukünftigen Vertreter könnten unter anderem in der unterschiedlichen Ausbildung der Berater liegen: So haben über $70 \%$ der Überleitungsfachkräfte und Hospizmitarbeiter, sowie $58 \%$ der Notare eine Schulung/Weiterbildung zur Beratung besucht, jedoch nur 24\% der Ärzte. Untersuchungen belegen, dass bereits kurze Schulungen Unsicherheiten im Beratungsgespräch reduzieren können (Detering et al. 2014). Auch in der Literatur wird auf den Bedarf nach Schulungen insbesondere für Ärzte hingewiesen (Schildmann und Winkler 2014; van Oorschot et al. 2012; Wandrowski et al. 2012). Möglicherweise könnten Schulungen das Bewusstsein für die genannten Zusammenhänge im Bereich der Stellvertretung schärfen sowie Unsicherheiten in der Gesprächsführung verringern und dadurch zu einer vermehrten Einbeziehung des Vertreters führen.

Eine Ermutigung zu diesem Schritt dürften die in der Studie ermittelten positiven Erfahrungen der anderen Berater sein.

\subsubsection{Fertig erstellte Patientenverfügungen}

Ein herausragender Befund der Studie ist die Feststellung, dass nur zwischen 29-51\% aller Beratungen mit einer fertig erstellten Patientenverfügung endeten. Dieses Ergebnis weicht wesentlich von der nach einem umfassenden Gesprächsprozess im Rahmen eines ACP Programmes vorliegenden Prävalenz von Patientenverfügungen ab (Hammes et al. 2010; In der Schmitten et al. 2014). Es unterstreicht jedoch das große Interesse an Informationen zum Thema Vorsorge. Unklar ist, ob die Verfügungen später zuhause fertig gestellt werden oder ob andere Faktoren, wie z.B. Unsicherheiten aufgrund ungeklärter Fragen die schriftliche Dokumentation der Wünsche verhindern. Eine bessere Kenntnis der Berater 
über mögliche Barrieren sowie Schulungen zur Beratung könnten hilfreich sein, um die Anzahl der fertiggestellten Patientenverfügungen zu erhöhen. Fraglich ist auch, ob es überhaupt in allen Fällen das Ziel der Berater ist, die Beratung mit einer unterschriebenen Patientenverfügung zu beenden. Mancher Berater könnte ein Autonomieverständnis dahingehend haben, dass der Ratsuchende alleine nochmals alles überdenken und das Dokument dann selbstständig fertigstellen soll. Zur Frage nach den Gründen für die bestehende Situation bei der Fertigstellung der Patientenverfügung wäre Forschung daher wünschenswert.

\subsubsection{Bezahlung der Beratung}

Die Fragen zur Bezahlung der Beratung wurden sehr unterschiedlich beantwortet. Der Großteil der nicht-juristischen Beratergruppen führte die Beratungen für den Ratsuchenden unentgeltlich durch. Fraglich ist, mit welcher Begründung und in welcher Höhe die anderen Berater dieser Gruppen dem Ratsuchenden die Beratung berechnen. Weiter wäre hilfreich zu erfahren, wie die Akzeptanz einer Bezahlung bei den Beratenen ist.

Überraschenderweise beriet auch ein knappes Drittel der Notare die Ratsuchenden unentgeltlich. Die Gründe hierfür kennenzulernen wäre unterstützend für die Weiterentwicklung der Beratung.

Bei Hausärzten und Notaren scheinen diejenigen, die kostenpflichtig beraten auch persönlich eine Vergütung dafür zu beziehen. Dagegen erhalten Überleitungsfachkräfte und Mitarbeiter von Hospizvereinen, von Einzelfällen abgesehen, keine direkt an die Beratung gekoppelte Vergütung.

Nur $32 \%$ der Ärzte und $12-15 \%$ der Mitglieder der anderen Beratergruppen, die persönlich eine Vergütung erzielten, hielten diese auch für angemessen. Somit war selbst ein Großteil derjenigen, die eine Vergütung für die Beratung erhielten, mit deren Höhe nicht zufrieden. Von 34\% der Ärzte wurde eine Vergütung zwischen 50-99 Euro, von 17\% der Ärzte 25-49 Euro als angemessen angesehen. Zu klären wäre die Frage, von wem die Kosten für die Beratung in der Zukunft getragen werden sollen: Weiter von vielen Beratern selbst, deren Einrichtungen, den Krankenkassen oder dem Ratsuchenden? Hinweise auf eine mögliche Finanzierung durch die 
gesetzliche Krankenversicherung enthält das während der Abschlussarbeiten an dieser Studie durch das Bundesministerium für Gesundheit herausgegebene „Eckpunktepapier zur Verbesserung der Hospiz- und Palliativversorgung in Deutschland“ (Bundesministerium 2014).

\subsection{Inhalte der Beratung}

Die Inhalte der Beratung unterschieden sich sowohl zwischen den verschiedenen Beratergruppen, als auch innerhalb der Gruppen zum Teil erheblich.

\subsubsection{Medizinische Situationen}

Während über $80 \%$ aller Befragten die Themen „Flüssigkeit und Ernährung am Lebensende“, sowie "Unheilbare, tödlich verlaufende Krankheit“ noch häufig oder fast immer aufgriffen, war das Beratungsverhalten in den anderen Bereichen deutlich diverser. So bearbeiteten beispielsweise alle Gruppen das Thema „Psychiatrische Erkrankungen“ weit weniger intensiv (24-55\%).

\subsubsection{Information über Notfallpläne}

Auffällig ist die Situation bei der Information über die Möglichkeit eines Notfallplanes: Die Notare erwähnten sie wenig ( $h / i=12 \%)$, wohingegen zwei Drittel der Mitarbeiter der Hospizvereine darüber informierten. Bei den anderen Beratern zeichneten sich jeweils ähnlich große Gruppen ab: Ein Teil informierte fast nie oder selten zu diesem Thema (n/s Überleitungsfachkräfte $34 \%$, Ärzte 51\%), der andere häufig oder fast immer (h/i Überleitungsfachkräfte 48\%, Ärzte 44\%). Gerade die Information über die Möglichkeit eines Notfallplanes sollte jedoch fester Bestandteil einer umfassenden Beratung sein (In der Schmitten et al. 2014; Schulte-Strathaus 2014; Wiese et al. 2008), um dem Ratsuchenden die Kenntnis und Nutzung dieser Vorsorgemöglichkeit zu ermöglichen. 


\subsubsection{Erarbeitung von Notfallplänen}

Die Erarbeitung von Notfallplänen für schwerkranke und/oder hochbetagte multimorbide Menschen wird zunehmend gefordert (Brokmann et al. 2014; In der Schmitten et al. 2011; Schulte-Strathaus 2014; Wiese et al. 2008). Auch niedergelassene Hausärzte wünschen sich eine verstärkte Verbreitung (Wiese et al. 2011b). Notfallpläne sind, z.B. unter dem Namen POLST $^{13}$ oder HAnNo, fester Bestandteil von ACP Programmen in den USA, Australien und Deutschland (Hammes et al. 2012; In der Schmitten et al. 2011).

Dieses Instrument der Vorsorge erstellten jedoch nur wenige Mitglieder der befragten Gruppen (h/i 0-45\%). Bei den Notaren (0\%) erscheint dies konsequent, da sie kaum die medizinischen Kompetenzen für die Erstellung eines Notfallplanes haben dürften. Bei den Überleitungsfachkräften mag die geringe Zahl $(\mathrm{h}=10 \%)$ daran liegen, dass sie diese Aufgabe allein bei den Ärzten sehen. Bemerkenswert ist jedoch, dass 74\% der Hausärzte fast nie oder selten Notfallpläne erarbeiteten, obwohl jeder Hausarzt ja auch mit Notfällen und dem Sterben seiner Patienten konfrontiert wird. Auch bei der Betrachtung der Antworten der Ärzte, die häufig Schwerkranke oder Menschen mit einer Lebenserwartung von unter 6 Monaten beraten, wurden von mehr als der Hälfte fast nie oder selten Notfallpläne erstellt. Hier ist fraglich, ob dieses Instrument bei den Mitarbeitern der an der Erstellung beteiligten Berufsgruppen bereits ausreichend bekannt ist und sie sich sicher genug in der Anwendung fühlen (Brokmann et al. 2014). Aufgrund der Tatsache, dass Notfallpläne im Ernstfall ohne weitere Prüfung direkt zur Anwendung gelangen (sollen), sind besondere Sorgfalt und medizinische Kompetenz bei ihrer Erstellung unerlässlich. In den genannten ACP-Programmen sind hierfür Voraussetzungen festgelegt, um Handlungssicherheit zu schaffen (Hammes et al. 2012).

${ }^{13}$ Die POLST ist kein reiner Notfallplan, sondern enthält daneben auch ärztliche Anordnungen über lebensverlängernde Maßnahmen (z.B PEG). 


\subsubsection{Hinweis auf die Möglichkeit einer Organspende}

Das Thema Organspende spielte in der vorliegenden Studie in der Beratung durch die Teilnehmer keine große Rolle. Zwischen 54-72\% der Mitglieder der Beratergruppen gaben selten oder fast nie einen Hinweis auf die Möglichkeit einer Organspende. Bei den Hausärzten wiesen nur 44\% häufig oder fast immer auf diese Möglichkeit hin, obwohl auch die Bundesärztekammer einen derartigen Hinweis empfiehlt (Bundesärztekammer 2013). Diese relativ geringen Häufigkeiten erstaunen, da die größte Gruppe der Ratsuchenden nach Kenntnis ihrer Berater gesund und damit sicher vielfach als Organspender geeignet war (Abb.3). Auch beinhalten viele der neueren Formulare zur Patientenverfügung das Thema Organspende, so dass es als häufiger Bestandteil der Beratung zu vermuten gewesen wäre. Möglicherweise wird jedoch die Besprechung von zwei „schweren Themen“ in einer Beratung als zu belastend oder zeitaufwendig empfunden. Nicht auszuschließen ist auch, dass sich der Organspendeskandal noch auf die Bereitschaft zur Ansprache auswirkt.

Bei der inhaltlichen Aufbereitung der Thematik Organspende zeichneten sich erhebliche Unterschiede innerhalb und zwischen den Gruppen ab (Abb. 20). Zwar sprachen die Ärzte nach ihren Aussagen das Thema Hirntod noch zu $67 \%$ häufig oder immer an. Alle anderen grundlegenden Themen der Organspende wurden von 4-37\% der Befragten der verschiedenen Gruppen nur selten oder fast nie einbezogen. Zusammenfassend lässt sich erkennen, dass der Informationsfluss in diesem Bereich insgesamt dringend verbessert werden sollte. Zumindest sollte im Zusammenhang mit gesundheitlicher Vorausplanung der mögliche aber leicht lösbare Konflikt zwischen Organspende und Patientenverfügung häufiger thematisiert werden (statt bisher nur von 15\%-36\% der Berater). Dadurch könnte der Ratsuchende das Bewusstsein für diese Frage entwickeln und eine für inn passende Entscheidung treffen.

\subsubsection{Hinweise zur Aufbewahrung der Patientenverfügung}

Die weit überwiegende Zahl der Berater (>81\%) gab Hinweise zur Aufbewahrung der Patientenverfügung. Sie scheinen sich damit der Bedeutung der Auffindbarkeit der Dokumente (Marckmann und In der Schmitten 
2013; Morrison et al. 1995; Sommer et al. 2012a; Weinick et al. 2008) bewusst zu sein und dies auch zu kommunizieren. Auf die Bedeutung der Aufbewahrung wird auch in den Empfehlungen der Bundesärztekammer, den Standards für die Beratung zu Patientenverfügungen und der Forschung hingewiesen (Bundesärztekammer 2013; May et al. 2005; Sommer et al. 2012). Der Auffindbarkeit der Dokumente wird in den ACP Programmen ebenfalls ein besonderer Stellenwert zugemessen, indem sie die Entwicklung von Routinen und Standards für die Aufbewahrung und Weitergabe der Papiere fordern (Silvester et al. 2013b). Betrachtet man die in dieser Studie benannten Hinweise im einzelnen, wird deutlich, wie sehr jede Berufsgruppe aus ihrer Profession heraus beriet: Hausärzte empfahlen hauptsächlich die Hinterlegung einer Kopie beim Arzt, Überleitungsfachkräfte eine Kopie in der Einrichtung (Heim), Notare das Zentrale Vorsorgeregister ${ }^{14}$. Lediglich die Mitarbeiter der Hospizvereine empfahlen hauptsächlich eine mündliche oder schriftliche Information des Vertreters. Eine erforderliche Information des Vertreters, der ja im Falle der Nichteinwilligungsfähigkeit des Vertretenen an der Behandlungsentscheidung teilnehmen muss, wurde insgesamt nur 39 mal angegeben. Daher ist davon auszugehen, dass noch erheblicher Bedarf sowohl qualitativ an Präzisierung der Hinweise zur Aufbewahrung aber auch quantitativ bei den Beratern, die bisher noch keine Hinweise geben, besteht.

\subsection{Umfeld der Beratung, Vernetzung}

\subsubsection{Verweis zur ergänzenden Beratung}

Einen Verweis zur ergänzenden Beratung durch andere an der Vorsorgeplanung beteiligte Berufsgruppen erteilten nach ihren Angaben 40-77\% der Mitglieder der befragten Gruppen häufig oder fast immer. Betrachtet man im Einzelnen welche Berufsgruppen aufeinander verwiesen, so zeigt sich, dass zwischen 65-89\% der Mitglieder der nicht-ärztlichen Beratergruppen eine ergänzende Beratung durch einen Arzt empfahlen. Diese

\footnotetext{
${ }^{14}$ Im ZVR wird nicht die Patientenverfügung selbst hinterlegt. Die Daten des ZVR sind nur den Gerichten zugänglich (Bundesnotarkammer 2013).
} 
Berater waren sich also des Bedeutung ärztlicher Beratung bewusst und förderten diese. Aus der Studie lässt sich nicht ablesen, inwieweit dem gegebenen Hinweis durch die Ratsuchenden gefolgt wird oder die Ärzte tatsächlich ergänzend beraten. Umfassende ACP Programme sehen eine Beratung durch medizinisch geschultes Personal in Zusammenarbeit mit einem Arzt vor (Hammes et al. 2010; In der Schmitten et al. 2014; Silvester et al. 2013a). Ein (abschließendes) Gespräch mit einem Arzt ist Kernbestandteil der best practice von ACP Programmen, um das Verständnis der niedergelegten Wünsche durch den Ratsuchenden nochmals zu überprüfen und seine Einwilligungsfähigkeit festzustellen (In der Schmitten et al. 2014; Silvester et al. 2013b). Bereits in den Beratungen zur Gestaltung der gesetzlichen Regelungen zur Patientenverfügung wurde auf die Notwendigkeit einer ärztlichen Beratung bei der Verfassung einer Patientenverfügung aufmerksam gemacht, auch wenn sie nicht zur Wirksamkeitsvoraussetzung wurde (Bundestag 2008). In der Literatur wird ebenfalls mehrfach darauf hingewiesen, dass es ohne ärztliche Beratung häufig nicht zu tragfähigen Patientenverfügungen kommt (Nauck et al. 2014; Schöffner et al. 2012). So kann die Verwendung von Formularen ohne medizinische Beratung zu erheblichen Inkongruenzen zwischen den niedergelegten und den tatsächlichen Wünschen der Menschen führen (Nauck et al. 2014; Schöffner et al. 2012). Nimmt man die Tatsache hinzu, dass zahlreiche Patientenverfügungen den konkreten Anwendungsfall nicht erfassen (Langer et al. 2013; Sommer et al. 2012), ist die Forderung zu unterstreichen, dass gerade medizinisches Wissen noch mehr in die Beratung einbezogen werden müsste. $\mathrm{Da}$ in Deutschland viele nicht medizinnahe Personen oder Organisationen in der Beratung zur Patientenverfügung aktiv sind, kommt der abschließenden Beratung durch einen Arzt ${ }^{15}$ nochmals eine besondere Bedeutung zu. Dieser Zusammenhänge sollten sich alle Beratergruppen bewusst sein, um eine stärkere Einbeziehung der Ärzte zu fördern.

\footnotetext{
15 insbesondere auch bei bereits schwer erkrankten oder hochbetagten, multimorbiden Menschen, bei denen eine Bestätigung der Einwilligungsfähigkeit sinnvoll sein könnte,
} 
Der von der Hälfte der Ärzte erfolgte Verweis an Notare zur ergänzenden Beratung ist zu begrüßen, wenn es sich um die Beratung zu Fragen der Vorsorgevollmacht, Betreuungsverfügung oder zu rechtlichen Aspekten der Patientenverfügung handelt. Für die rechtswirksame Erstellung einer Patientenverfügung und einer Vorsorgevollmacht in Gesundheitsfragen ist gesetzlich keine notarielle Beratung oder Beglaubigung/Beurkundung vorgeschrieben ${ }^{16} 17$.

Eine sehr wichtige Rolle spielten Hospiz- und Betreuungsvereine bei der ergänzenden Beratung. $30-71 \%$ der Ärzte, Überleitungsfachkräfte und Hospizmitarbeiter verweisen auf sie. Bemerkenswert ist dagegen die anscheinend geringe Nutzung der nicht-ärztlichen Kompetenzen der anderen Berater durch die Notare $(0-12 \%)$. Gründe dafür sind bislang nicht bekannt.

Es lässt sich der Studie somit entnehmen, dass sich viele Berater der Erforderlichkeit einer ergänzenden Beratung durch Ärzte und eventuell andere Berufsgruppen in bestimmten Fällen bewusst sind. Das Wissen um die gegenseitigen Kompetenzen, aber auch deren Grenzen sollte bei allen Beratern gefördert werden, um die bestmögliche, umfassende und praxistaugliche gesundheitliche Vorausplanung des Ratsuchenden zu ermöglichen. Diese Anforderung wird aber noch nicht umfassend (alle Berater wissen um die Kompetenzen aller anderen Berater und verweisen bei Bedarf auf diese) umgesetzt.

\footnotetext{
${ }^{16}$ Durch eine Beglaubigung kann jedoch die Identität des Unterschreibenden mit dem Aussteller des Dokumentes bestätigt werden ( $\$ 129$ BGB). Mit einer Beurkundung können auch Feststellungen über die Geschäfts- bzw. Einwilligungsfähigkeit des Ratsuchenden verbunden werden ( $\$ 11$ BeurkG).

${ }^{17}$ Nach deutschem Recht sind jedoch einige über die Gesundheitssorge hinausgehende Vertretungsmaßnahmen nur mit einer notariell beurkundeten Vollmacht (z.B. Verkauf von Grundstücken, Handelsgeschäfte) möglich. Eine tragfähige Vorausplanung beinhaltet, wenn vom Ratsuchenden gewünscht, auch die Benennung und ausreichende Legitimierung eines Vertreters für die anstehenden Aufgaben. Es kann Fälle geben, in denen der Vertreter z.B. das Haus des Vertretenen verkaufen oder Handelsgeschäfte weiterführen muss, um eine ausreichende gesundheitliche Versorgung des Vertretenen finanzieren zu können. Insbesondere in derartigen Fällen können Fragen auftreten, deren korrekte Beantwortung und Umsetzung explizit rechtliche Kenntnisse und Befugnisse (z.B. Beglaubigung, Beurkundung) voraussetzt.
} 


\subsubsection{Einbeziehung der Berater bei medizinischen Entscheidungen}

Die Einbeziehung der Berater bei medizinischen Entscheidungen im Falle der Einwilligungsunfähigkeit des Patienten erfolgte je nach Beratergruppe unterschiedlich häufig. Obwohl die Bundesärztekammer eine Einbeziehung des behandelnden Arztes empfiehlt ${ }^{18}$ (Bundesärztekammer 2013), geschah dies nur bei $68 \%$ der befragten Hausärzte. Dies könnte bedeuten, dass die Patienten der anderen 32\% der Hausärzte in Einrichtungen (z.B. Krankenhaus) versterben, in denen andere Ärzte den Patientenwillen ohne Einbeziehung der Hausärzte ermitteln.

Die anderen Beratergruppen wurden noch weniger bis gar nicht involviert (0-60\%). Dieses verwundert insbesondere bei den Mitarbeitern der Hospizvereine die, ebenso wie vielfach die Hausärzte, die Patienten, in ihrer letzten Lebenszeit begleiten und somit häufig gut kennen dürften. Damit wird möglicherweise bei den Beratern vorhandenes Wissen um die Wünsche des Patienten nicht genutzt. Fraglich ist zum einen, wovon die Einbeziehung abhängt ${ }^{19}$ und welchen Beitrag sie zur Entscheidungsfindung tatsächlich leisten kann. Zum anderen ist zu überlegen, ob aus der Einbeziehung Konsequenzen, z.B. für die Dokumentation der Beratung, abgeleitet werden sollten.

\subsection{Wünsche der Berater}

\subsubsection{Wünsche an die Weiterentwicklung der Beratung}

Die von den Beratern geäußerten Wünsche zur verstärkten Zusammenarbeit bzw. zum Austausch mit anderen Berufsgruppen unterstreichen das Interesse an einer besseren Vernetzung insbesondere mit Ärzten. Ärzte wurden von überwiegenden Teilen der nichtärztlichen Berater als Wunschpartner für eine verstärkte Zusammenarbeit angegeben (62-80\%).

\footnotetext{
18 und auch die Ärzte selbst viel Wert auf eine ausreichende Information durch Hinterlegung einer Kopie der Patientenverfügung bei innen legen (Abb. 13),

${ }_{19}$ Möglicherweise liegt zwischen der Beratung zur Patientenverfügung und der Entscheidungssituation auch ein längerer Zeitraum, der eine Erinnerung schwierig macht.
} 
Großes Interesse bestand ebenfalls am intensivierten Austausch mit den Betreuern (8-55\%) und den Hospizvereinen (15-40\%).

Insgesamt wünschten sich am häufigsten die Mitarbeiter der Hospizvereine eine verstärkte Zusammenarbeit mit anderen Berufsgruppen. Bei Ärzten und Notaren hatte jeweils circa ein Viertel der Berater keinen diesbezüglichen Wunsch. Fraglich ist, ob es bei diesen Personen bereits eine ausreichende Vernetzung gibt, oder ob sie sich der Notwendigkeit und der Chancen nicht bewusst sind.

Die vorliegende Studie belegt, dass große Teile der Berater für sich Bedarf an mehr Informationen, Austausch oder Zusammenarbeit in bestimmten Themenbereichen sehen. Bei den Ärzten stehen hier, auch noch 5 Jahre nach der gesetzlichen Regelung der Patientenverfügung, rechtliche Fragen im Vordergrund. Über die Hälfte der Ärzte (59\%) äußerte ihren Hauptbedarf in diesem Bereich, gefolgt von ethischen Fragen (37\%). Ähnlich ist die Situation bei den Überleitungsfachkräften (52\% rechtliche und $48 \%$ ethische Fragen).

Diese Aussagen decken sich mit den in der Literatur beschriebenen Unsicherheiten oder dem Schulungsbedarf von Ärzten und medizinischen Mitarbeitern in rechtlichen und ethischen Fragen (Cartwright et al. 2013; De Vleminck et al. 2014; Stone et al. 2013; Wandrowski et al. 2012; Wiese et al. 2011a).

Notare wünschten sich primär ein Feedback aus der Anwendungspraxis (62\%). Ein derartiges Feedback benötigen die Notare, um sowohl ihre Beratung, als auch die von innen erstellten Formulare weiterentwickeln zu können. Nachdem über die Hälfte der Notare dieses Feedback als hilfreich ansah und gleichzeitig großen Informationsbedarf zu medizinischen Fragen hatte, läge eine große Chance zu einem beidseitig befruchtenden Austausch in der intensiveren Vernetzung mit den Ärzten. Der dadurch mögliche Wissenstransfer könnte maßgeblich zur Verbesserung der Beratung beider Berufsgruppen beitragen.

Bei den Mitarbeitern der Hospizvereine verteilte sich das Interesse auf alle zur Auswahl gestellten Bereiche.

Nicht unerhebliche Teile der Beratergruppen (5-25\%) äußerten, keinen Wunsch nach mehr Information/Austausch/Zusammenarbeit zu haben 
Fraglich ist, ob hier bereits alle Wünsche erfüllt sind, oder ob, wie es Wandrowski bei Ärzten beschreibt (Wandrowski et al. 2012), eine Diskrepanz zwischen der Einschätzung des eigenen Wissens und dem tatsächlichen Wissen vorliegt.

Die gewünschte Verbesserung der Zusammenarbeit/ Information sahen alle nichtjuristischen Beratergruppen hauptsächlich in der Zusammenarbeit im Einzelfall (46-65\%). Auch ein knappes Viertel der Notare (23\%) wünschte diese Weiterentwicklung der Beratung zur Patientenverfügung. Diese Aussagen lassen sich als Hinweis auf die Bereitschaft zur Entwicklung einrichtungsübergeifender, strukturierter regionaler Netzwerke deuten, wie es auch in den Standards zur Beratung zur Patientenverfügung (May et al. 2005) und von ACP Programmen (Wilkinson 2011; In der Schmitten et al. 2014) gefordert wird.

Mit deutlichem Abstand benannten die Berater an zweiter Stelle der Verbesserungsmöglichkeiten Vorträge (23-35\%), gefolgt von regelmäßigen Treffen der Beratergruppen (8-30\%).

\subsection{Erwerb der für die Beratung benötigten Kenntnisse}

Die für die Beratung benötigten Kenntnisse erwarben 58-75\% der nichtärztlichen Berater durch Schulung oder Weiterbildung. Hier bestand demnach erhebliche Bereitschaft, sich speziell zur Vorsorgethematik weiterzubilden und nicht alleine auf ein Selbststudium (38-62\%) zu vertrauen. Bei den Ärzten erklärte dagegen weniger als ein Viertel der Befragten (24\%), eine Weiterbildung/Schulung besucht zu haben. Stattdessen eigneten sie sich zu 79\% ihr Wissen im Selbststudium an. In der Literatur gibt es zahlreiche Forderungen nach Schulungen der Berater (Knauss 2013; Schildmann und Winkler 2014; Schöffner et al. 2012). In etablierten ACP Programmen gehört die Schulung der beratenden Personen zur best practice und somit den Voraussetzungen eines erfolgreichen Gesprächsprozesses (Silvester et al. 2013b; Wilkinson 2011). Auch im Hinblick auf die bisherigen Ergebnisse dieser Studie ist fraglich, ob allein ein Selbststudium ausreicht, die für die Beratung nötigen Kompetenzen zu erwerben, Unsicher- 
heiten abzubauen und eine qualitativ hochwertige Beratung anzubieten. Einige Berater äußerten in dem freien Feld "Persönliches Anliegen" explizit den Wunsch nach einer Verbesserung des Wissenstandes der Ärzte. Daher wäre es wünschenswert, bei allen Beratergruppen insbesondere aber bei den Ärzten, die den größten Nachholbedarf zu haben scheinen, verstärkt Weiterbildungen anzuregen. Wie die Ergebnisse von Detering zeigen (Detering et al. 2014), verringern selbst kurze Schulungen Unsicherheiten und haben einen deutlich positiven Effekt auf die Beratung.

Die von den Befragten besuchten Weiterbildungen erfolgten bei ca. einem Drittel der jeweiligen Gruppenmitglieder anhand bestimmter Formulare, bei den Mitarbeitern der Hospizvereine waren es sogar $60 \%$. Primär wurden dazu die Formulare des Bayerischen Staatsministeriums der Justiz (23-55\%), gefolgt von den Textbausteinen des Bundesjustizministeriums $(12-24 \%)$ eingesetzt. Alle anderen Formulare/Textbausteine wurden weit weniger häufig verwendet. Aus diesen Angaben lässt sich erneut die herausragende Bedeutung des Formulars des Bayerischen Ministeriums der Justiz in der untersuchten Region ablesen.

\subsection{Zusammenfassung der Diskussion}

Zusammenfassend lässt sich feststellen, dass die in der Studie erfasste Beratungssituation der best practice umfassender ACP Programme aufgrund der Heterogenität der Beratungen innerhalb und zwischen den beratenden Gruppen vielfach noch nicht vollständig entspricht. Zu nennen sind hier insbesondere das proaktive Angebot einer Beratung, die stärkere Einbeziehung von (geschulten) Ärzten, aber auch zukünftigen Vertretern in die Beratung. Daneben sind bestimmte Mindestinhalte der Beratung, die bei Bedarf auch eine Notfallplanung umfassen noch nicht immer gegeben. Der Umfang der Studie ließ eine abschließende Klärung der Ursachen für die unterschiedlichen Beratungsstrukturen und -inhalte nicht zu: Sind dem Berater einige der wichtigen Themen nicht als relevant bewusst? Fühlt er sich inhaltlich bei einigen Themen zu unsicher, um sie anzusprechen? Glaubt er, sie haben für den konkret zu Beratenden keine Bedeutung? Oder geht er, wie Hancock es zum ärztlichen Gespräch zu End-of-Life 
Fragen beschreibt, von einem zu hohen Vorverständnis und einem zu geringen Informationsbedarf der Ratsuchenden aus (Hancock et al. 2007)? Welche Faktoren aus dem Bereich des Ratsuchenden, welche aus dem des Beraters fließen bei der Gestaltung des Gespräches mit ein?

Weitere Forschung zu den Ursachen des unterschiedlichen Beraterverhaltens wäre daher hilfreich, um eventuelle Barrieren für die Weiterentwicklung der Beratung identifizieren und bearbeiten zu können.

Aufgrund der Diversität der Angaben innerhalb der Gruppen ist zur Zeit für die Ratsuchenden intransparent und unklar, welchen Beratungsinhalt die jeweiligen Beratergruppen anbieten, sie haben keine klaren Beratungsprofile. Diese Unklarheit kann als starkes Argument für eine Erarbeitung bzw. Weiterentwicklung (May et al. 2005) von Qualitätskriterien für die Beratung durch die verschiedenen Beratergruppen gewertet werden. Derartige Kriterien wären ein Schritt auf dem Weg zu einer gleichmäßig hohen Qualität von Beratung.

\subsection{Limitationen}

Ziel der vorliegenden Studie war es, einen ersten Einblick in die Beratungssituation zur Patientenverfügung in einer Region zu geben und daraus Perspektiven für die Weiterentwicklung abzuleiten. Durch die Begrenzung der Umfrage auf einige Beratergruppen ist eine Verallgemeinerung der Ergebnisse auf die gesamte Beratungssituation der Region München nicht möglich. Die geringe Teilnahme der angeschriebenen Ärzte spiegelt die bereits in der Literatur beschriebene Zurückhaltung von Hausärzten bei Umfragen wieder (Güthlin et al. 2012). Daher sind die Aussagen der Ärzte nur bedingt auf die anderen Ärzte der Region München übertragbar. Zudem haben sich möglicherweise nur die Ärzte beteiligt, die ein besonderes Interesse an der Vorsorgethematik haben. Sollte diese Vermutung zutreffen, könnte sich die Gesamtsituation der ärztlichen Beratung ungünstiger als in dieser Studie erfasst, darstellen. Letztere Vermutung könnte auch auf die Notare und Überleitungsfachkräfte zutreffen.

Durch die leicht variierenden Rekrutierungs- bzw. Zugangswege zum Fragebogen sind freie Selektionseffekte nicht auszuschließen. 
Die in dieser Studie ermittelten Ergebnisse lassen erste Aussagen über den Inhalt und die Vielfalt der angesprochenen Themen der Beratung zu. Die Studie macht nur teilweise Aussagen dazu, mit welcher Tiefe und Sachkenntnis diese Themen dann durch die Berater im Einzelnen bearbeitet werden. Auch der Frage nach den Gründen für ein bestimmtes Verhalten von Beratern und Ratsuchenden konnte durch den begrenzten Umfang der Studie in der Umfrage nicht intensiv nachgegangen werden. 


\section{Ausblick: Perspektiven für die Weiterentwicklung der Beratung zur Patientenverfügung}

Aus den vorliegenden Ergebnissen lassen sich folgende Hinweise für die Weiterentwicklung der Beratung zur Patientenverfügung ableiten.

5.1 Vermehrte Initiative der Berater, aktives Beratungsangebot Die Beratung zur Patientenverfügung sollte vermehrt aktiv durch die Berater angeboten und nicht überwiegend der Initiative des zu Beratenden oder seiner Angehörigen überlassen bleiben. Wie zur best practice der ACP Programme gehörig, sollten die Berater „aufsuchend“ tätig werden, d.h. von sich aus auf die zu Beratenden zugehen und den Gesprächsprozess anbieten. Die Begründung für eine derartige Weiterentwicklung der Beratung findet sich in folgenden Ergebnissen der Studie:

- Die Berater selbst halten überwiegend ein routinemäßiges Angebot für sinnvoll (Abb. 28).

- Auch (noch) gesunde Menschen haben vielfach ein Interesse an Vorsorge (Abb. 4).

- Neben der hauptsächlich ratsuchenden Gruppe der 61-80jährigen sind auch bereits jüngere Menschen an Vorsorgemöglichkeiten interessiert (Abb. 5).

- Fraglich ist, ob schwer erkrankte Menschen bereits ausreichend auf die Möglichkeiten der Vorsorge angesprochen werden (4.1.1, 4.1.13).

- Fraglich ist, ob das Beratungsbedürfnis hochbetagter Menschen bereits ausreichend erfasst ist $(4.1 .1,4.1 .12)$.

Die Aufgabe zur Schaffung vermehrter aktiver Beratungsangebote richtet sich zunächst an die Mitglieder aller Beratergruppen, in besonderem Masse aber an die Hausärzte. Die Hausärzte scheinen nach den Ergebnissen der vorliegenden Untersuchung die größte Zurückhaltung bei der Initiative zu haben, obwohl sie aufgrund ihrer medizinischen Kompetenzen die 
fachlich geeigneten Personen sind. Gleichzeitig sehen sie auch selbst einen hohen Beratungsbedarf und sind nach den Angaben in der Literatur vielfach der von den Patienten gewünschte Gesprächspartner. Den anderen Beratergruppen kommt ebenfalls ein erheblicher Einfluss bei der Initiierung von Beratungsangeboten zu, da sie jeweils einen besonderen $\mathrm{Zu}$ gang zu bestimmten Personengruppen haben.

Eine besondere Bedeutung bei der Weiterentwicklung der Vorsorgeangebote haben Institutionen, wie z.B. Altersheime. Die Mitarbeiter dieser Einrichtungen sehen den Bedarf, können das individuelle Bedürfnis des jeweiligen Bewohners nach Vorausplanung ermitteln und ein Angebot zur Beratung veranlassen. Voraussetzung für derartige Angebote ist, dass die Einrichtung eine derartige Initiative ihrer Mitarbeiter wünscht, fördert und die dafür erforderlichen Ressourcen zur Verfügung hat. Einige Einrichtungen haben diese Aufgabe schon angenommen, indem sie systematisch Beratungen durch die Überleitungsfachkräfte anbieten. Daneben wäre ein weiterer Ausbau der Vorausplanung und geregelte Kooperationen mit den Hausärzten in diesem Bereich wünschenswert.

Flankiert werden sollte die vermehrte Initiative der einzelnen Berater durch verstärkte Öffentlichkeitsarbeit, um so bestehende Barrieren und/oder Fehlvorstellungen bei der Bevölkerung weiter abzubauen.

Unterstützend könnten auch die entsprechenden Vertretungen der verschiedenen Beratergruppen, wie z.B. die Ärztekammer, die Bundesnotarkammer tätig werden, indem sie in ihren Veröffentlichungen und Schulungen auf die geschilderten Zusammenhänge hinweisen.

5.2 Verstärkte Einbeziehung zukünftiger Vertreter in die Beratung

Der Ratsuchende sollte zunächst bei der Wahl eines geeigneten Vertreters, der willens und in der Lage ist, die Vertretung zu übernehmen, unterstützt werden. Sofern der Ratsuchende einen zukünftigen Vertreter benennen kann, sollte dieser vermehrt in die Beratung zur Patientenverfügung einbezogen werden. Diese Perspektive der Weiterentwicklung stützt sich auf folgende Argumente: 
- Die Ergebnisse der Studie zeigen, dass bei weitem noch nicht alle Berater immer auf diese Möglichkeit hinweisen, und die Vertreter nicht regelmäßig an den Beratungen teilnehmen ( Abb. 17).

- Der Großteil der Berater bewertete die Einbeziehung des Vertreters als hilfreich (Abb. 18).

- Der Vertreter sollte die Vorstellungen des zu Vertretenden kennen, um so nahe wie möglich an dessen Wünschen orientiert entscheiden und die damit verbundene Belastung so gut wie möglich tragen zu können (4.1.7).

Es spricht viel dafür, ihn spätestens beim zweiten Gespräch zur Patientenverfügung mit einzubeziehen. So könnte der Kommunikationsprozess zwischen dem Ratsuchenden und seinem Vertreter unterstützt und sichergestellt werden, dass der Vertreter überhaupt von seiner Aufgabe, den Wünschen und Wertvorstellungen des zu Vertretenden weiß. Der Einwand, die Beratung diene zunächst nur der Entscheidungsfindung des Ratsuchenden selbst, trägt nur begrenzt, da es immer einer Person bedarf, die diese Entscheidungen kennt, mitträgt und umsetzt. Sofern der Ratsuchende eine Person beauftragt, sollte diese sich daher in der Regel frühzeitig mit ihrer Aufgabe vertraut machen können.

\subsection{Mindestanforderungen an Beratungsinhalt}

Innerhalb der beratenden Gruppen oder auch gruppenübergreifend sollten (verbindliche) Mindestinhalte, Standards oder Guidelines der Beratung formuliert werden. Diese Anregung beruht auf folgenden Erkenntnissen:

- Die Häufigkeit, mit der die verschiedenen in der Umfrage benannten Themen Inhalt der Beratung wurden, ist sowohl innerhalb der Gruppen, als auch zwischen den Gruppen sehr unterschiedlich (3.2).

- Es gibt einige grundlegende Themen, die nicht bei allen Beratungen (fast) immer angesprochen wurden, aber wegen ihrer Bedeutung 
angesprochen werden sollten (Fragen der Vertretung inklusive Angebot der Einbeziehung des Vertreters, Aufbewahrung der Vorsorgedokumente, Möglichkeit Notfallplan etc.) (3.2).

- Bei der derzeitigen Beratungspraxis besteht damit die Gefahr, dass die Ratsuchenden sehr unterschiedliche Beratungsangebote erhalten $^{20}$, ohne dass dieses nach außen transparent ist.

Daher sollte ein Themenkatalog gestaltet und durch alle Berater im Beratungsgespräch systematisch aufgegriffen werden, um die Mindestqualität einer Beratung zu gewährleisten. Sollten einige Gruppen bestimmte Schwerpunkte/Expertisen in ihrer Beratungspraxis haben (z.B. Beratung von Hochbetagten, Beratung ausschließlich anhand bestimmter, z.B. weltanschaulich geprägter Formulare, Vernetzung mit anderen Beratern) wäre es für die Ratsuchenden hilfreich, wenn auch diese Beratungsprofile formuliert und transparent gemacht würden. Auf dieser Basis können die Ratsuchenden einen Berater finden, der ihren Bedürfnissen entspricht.

\subsection{Notfallplanung}

Die Kenntnis der Berater und die Information der Ratsuchenden über die Bedeutung dieses Vorsorgeinstruments sollte weiter gefördert werden. Die Studie belegt, dass in diesem Bereich Handlungsspielräume bestehen:

- Informationen über die Möglichkeit eines Notfallplanes wurden nur von $44-65 \%$ (h/i) der Berater, bei den Notaren noch seltener $(\mathrm{h} / 112 \%)$ gegeben (Abb. 15).

- Die befragten Berater, insbesondere die Ärzte (h/i24\%) waren wenig an der Erstellung von Notfallplänen beteiligt (Abb.16).

Eine rechtzeitige und umfassende Notfallplanung sollte fester Bestandteil der Beratung durch Ärzte bei Schwerkranken und/oder hochbetagten multimorbiden Menschen werden, um ungewünschte Krankenhauseinweisun-

${ }^{20}$ Dieses zeigt sich z. B. bei den Hinweisen zur Aufbewahrung der Patientenverfügung (Abb.13) oder der unterschiedlichen Einbeziehung zukünftiger Vertreter (Abb.16) 
gen und Therapien zu vermeiden, sowie Handlungssicherheit für alle an der Versorgung des Betroffenen Beteiligten zu schaffen.

\subsection{Finanzierung der Beratung}

Eine qualitativ hochwertige Beratung erfordert Zeit, eine gute Ausbildung sowie die Bereitschaft zur Weiterbildung. Die Schaffung dieser Voraussetzungen sollte durch eine ausreichende Finanzierung der Beratung gefördert werden. Die Studie zeigt, dass im Bereich der Finanzierung noch Defizite bestehen:

- Ein Großteil der Berater berät unentgeltlich (Abb.31,32).

- Viele Teilnehmer, insbesondere die Ärzte, empfinden die derzeitige finanzielle Situation als unangemessen (Abb.33).

Wünschenswert wäre die (Weiter-) Entwicklung und Umsetzung von Konzepten, wie die Beratung insbesondere durch Ärzte, aber auch durch Mitarbeiter z.B. der Institutionen Hospizverein und Altenheim finanziert werden kann. Dass die Politik die Bedeutung der Weiterentwicklung der Beratung und deren Finanzierung mittlerweile erkannt hat, lässt sich Punkt 6 des Eckpunktepapiers des Bundesministeriums für Gesundheit entnehmen (Bundesministerium 2014).

\subsection{Schulungen}

Die Ergebnisse dieser Studie legen einen Zusammenhang zwischen einer Weiterbildung, die über das Selbststudium hinausgeht und der Qualität der Beratung nahe. Zur Reduzierung von Unsicherheiten oder Barrieren im Bereich der Beratung wären daher praxisorientierte Schulungen insbesondere zu folgenden Themen sinnvoll:

- Initiierung und Durchführung eines Gespräches zur Vorausplanung

- Barrieren von Ratsuchenden und Beratern

- Effekte der Beratung

- Mindestinhalte der Beratung 
- Bedeutung und Erstellung von Notfallplänen

- Rechtliche, medizinische und ethische Fragestellungen

\subsection{Forschung}

Die vorliegende Arbeit weist auf etliche Bereiche hin, in denen weitere Forschung für die Weiterentwicklung der Beratung wichtig wäre:

- Identifizierung und Bearbeitung von Barrieren von Ratsuchenden, Angehörigen und Beratern bei der Initiierung der Beratung (Abb.1,2).

- Ermittlung von Gründen, warum die Patientenverfügung nicht häufiger innerhalb der Beratung fertiggestellt wird (Abb.12).

- Ermittlung von Gründen für die Nichteinbeziehung von zukünftigen Vertretern in das Beratungsgespräch (Abb.17).

- Ermittlung von Gründen für die Einbeziehung/Nichteinbeziehung von Beratern in die Entscheidungsfindung bei Nichteinwilligungsfähigkeit und Konsequenzen daraus für die Beratung (Abb. 22).

- Sinnhaftigkeit, Möglichkeiten der Entwicklung und Einführung eines einheitlichen Formulars für die Patientenverfügung, insbesondere eines einheitlichen Notfallplanes (Abb. 9-11, 30).

Wie die hier aufgeführten Perspektiven der Weiterentwicklung der Beratung zeigen, handelt sich dabei nicht nur um eine Aufgabe, die sich dem einzelnen Berater stellt. Eine Weiterentwicklung der Beratung hin zu einer tragfähigen Vorsorge ist vielmehr nur möglich, wenn sich auch die jeweiligen Berufsvertretungen und Institutionen dieser ethisch gebotenen Aufgabe bewusst sind und die Weiterentwicklung fördern. Sie kann nur gelingen, wenn sie durch die beruflichen Organisationen, die versorgenden Institutionen und die Öffentlichkeit gemeinsam unterstützt wird. 


\section{Literaturverzeichnis}

Alano GJ, Pekmezaris R, Tai JY, Hussain MJ, Jeune J, Louis B et al. Factors influencing older adults to complete advance directives. Palliat Support Care 2010;8(3):267-75.

Almack K, Cox K, Moghaddam N, Pollock K, Seymour J. After you: conversations between patients and healthcare professionals in planning for end of life care. BMC Palliat Care 2012(11):15.

Amjad $\mathrm{H}$, Towle V, Fried T. Association of Experience with Illness and End-of-life Care with Advance Care Planning in Older Adults. J Am Geriatr Soc 2014;62(7):1304-9.

Azoulay E, Pochard F, Chevret S, Arich C, Brivet F, Brun F et al. Family participation in care to the critically ill: opinions of families and staff. Intensive Care Med 2003;29(9):1498-504.

Barnes KA, Barlow CA, Harrington J, Ornadel K, Tookman A, King $M$ et al. Advance care planning discussions in advanced cancer: analysis of dialogues between patients and care planning mediators. Palliat Support Care 2011;9(1):73-9.

Becker M, Jaspers B, King C, Radbruch L, Voltz R, Nauck F. Did you seek assistance for writing your advance directive? A qualitative study. Wien Klin Wochenschr 2010;122(21-22):620-5.

Bienwald W, Sonnenfeld S, Hofmann B. Betreuungsrecht Kommentar. Bielefeld: Gieseking 2011.

Bortz J, Döring N. Forschungsmethode und Evaluation: für Human- und Sozialwissenschaftler. Heidelberg: Springer Medizin 2006.

Bosshard G, Nilstun T, Bilsen J, Norup M, Miccinesi G, van Delden JJ et al. Forgoing treatment at the end of life in 6 European countries. Arch Intern Med 2005;165(4):401-7.

Bravo G, Arcand M, Blanchette D, Boire-Lavigne AM, Dubois MF, Guay M et al. Promoting advance planning for health care and research among older adults: a randomized controlled trial. BMC Med Ethics 2012(13):1.

Brinkman-Stoppelenburg A, Rietjens JA, van der Heide A. The effects of advance care planning on end-of-life care: A systematic review. Palliat Med 2014(8):100025.

Brokmann JC, Grutzmann T, Pidun AK, Gross D, Rossaint R, Beckers SK et al. Advance directives in prehospital emergency treatment : prospective questionnaire-based analysis. Anaesthesist 2014;63(1):23-31.

Bundesärztekammer. Empfehlungen der Bundesärztekammer und der Zentralen Ethikkommission bei der Bundesärztekammer Umgang mit Vorsorgevollmacht und Patientenverfügung in der ärztlichen Praxis. Dtsch Arztebl International 2013;110(33-34):1580-5. 
Bundesministerium für Gesundheit. Eckpunktepapier: Verbesserung der Hospizund Palliativversorgung in Deutschland. http://www.bmg.bund.de/ministerium/meldungen/verbesserung-hospiz-undpalliativversorgung.html (Zugriff am 3.12.2014)

Bundesnotarkammer. Zentrales Vorsorgregister der Bundesnotarkammer. http://www.vorsorgeregister.de/_downloads/Jahresberichte/2013JBZVR.pdf (Zugriff am 3.12.2014)

Bundestag. Drucksache des Bundestages 16/8842. http://dip21.bundestag.de/dip21/btd/16/084/1608442.pdf (Zugriff am 3.12.2014)

Burchardi N, Rauprich O, Hecht M, Beck M, Vollmann J. Discussing living wills. A qualitative study of a German sample of neurologists and ALS patients. J Neurol Sci 2005;237(1-2):67-74.

Carr D. "I don't want to die like that ...": the impact of significant others' death quality on advance care planning. Gerontologist 2012;52(6):770-81.

Cartwright C, Montgomery J, Rhee J, Zwar N, Banbury A. Medical Practitioners' Knowledge and Self-reported Practices of Substitute Decision-making and Implementation of Advance Care Plans. Intern Med J 2014; 44(23):234-9

Clayton J. Advance Care Planning discussions in Australia: the development of clincal practice guidelines. In: Thomas K, Lobo B (Hg.) Advance Care Planning in End of Life Care. Oxford: Oxford University Press 2011, S. 232-250.

Collins $\mathrm{M}$, Lehane E. Perspectives on death, dying and advance care planning from patients receiving haemodialysis. J Ren Care 2013;39(1):5-11.

Davies E, Higginson IJ. Better Palliative Care for Older Pepole. Copenhagen: World Health Organization. Regional Office for Europe 2004.

De Vleminck A, Houttekier D, Pardon K, Deschepper R, Van Audenhove C, Vander Stichele $\mathrm{R}$ et al. Barriers and facilitators for general practitioners to engage in advance care planning: a systematic review. Scand J Prim Health Care 2013;31(4):215-26.

De Vleminck A, Pardon K, Beernaert K, Deschepper R, Houttekier D, Van Audenhove $\mathrm{C}$ et al. Barriers to advance care planning in cancer, heart failure and dementia patients: a focus group study on general practitioners' views and experiences. PLoS One 2014;9(1):e84905.

Detering K, Silvester W, Corke C, Milnes S, Fullam R, Lewis V et al. Teaching general practitioners and doctors-in-training to discuss advance care planning: evaluation of a brief multimodality education programme. BMJ Support Palliat Care 2014;4(3):313-21.

Detering KM, Hancock AD, Reade MC, Silvester W. The impact of advance care planning on end of life care in elderly patients: randomised controlled trial. BMJ 2010;340:c1345.

Dodegge G. Der Schutz des freien Willens durch die Rechtsinstitute Betreuung, Vorsorgevollmacht, Betreuungs-und Patientenverfügung. Familie, Partnerschaft, Recht 2008:595-91. 
Dörner K. Autonomie am Lebensende. Zeitschrift für Palliativmedizin 2005;6:109111.

Ernst J, Krauß O, Schwarz R. Die Einbeziehung von Patienten in medizinische Entscheidungsprozesse. Der Onkologe 2006(12):48-54.

Fagerlin A, Ditto PH, Danks JH, Houts RM, Smucker WD. Projection in surrogate decisions about life-sustaining medical treatments. Health Psychol 2001;20(3):166-75.

Fagerlin A, Schneider CE. Enough. The failure of the living will. Hastings Cent Rep 2004;34(2):30-42.

Forschungsgruppe Wahlen Telefonfeld (FGW). Sterben in Deutschland - Wissen und Einstellungen zum Sterben. Deutscher Hospiz- und PalliativVerband e.V.; 2012. http://www.dhpv.de/tl_files/public/Ueber Uns/Forschungsprojekte/201208_Bevoelkerungsumfrage_DHPV_Grafiken.pdf

Fromme EK, Zive D, Schmidt TA, Cook JN, Tolle SW. Association between Physician Orders for Life-Sustaining Treatment for Scope of Treatment and inhospital death in Oregon. J Am Geriatr Soc 2014;62(7):1246-51.

Gordy S, Klein E. Advance directives in the trauma intensive care unit: Do they really matter? Int J Crit IIIn Inj Sci 2011;1(2):132-7.

Gozalo P, Teno JM, Mitchell SL, Skinner J, Bynum J, Tyler D et al. End-of-life transitions among nursing home residents with cognitive issues. $\mathrm{N}$ Engl $\mathrm{J}$ Med 2011;365(13):1212-21.

Güthlin C, Beyer A, Erler A. Rekrutierung von Hausarztpraxen für Forschungsprojekte. Zeitschrift für Allgemeinmedizin 2012;88(4):173-81.

Gunderson Health System. Respecting Choices http://www.gundersenhealth.org/respecting-choices (Zugriff am 3.12.2014)

Hammes BJ, Rooney BL, Gundrum JD. A comparative, retrospective, observational study of the prevalence, availability, and specificity of advance care plans in a county that implemented an advance care planning microsystem. J Am Geriatr Soc 2010;58(7):1249-55.

Hammes BJ, Rooney BL, Gundrum JD, Hickman SE, Hager N. The POLST program: a retrospective review of the demographics of use and outcomes in one community where advance directives are prevalent. J Palliat Med 2012;15(1):7785.

Hancock K, Clayton JM, Parker SM, Walder S, Butow PN, Carrick S et al. Discrepant perceptions about end-of-life communication: a systematic review. J Pain Symptom Manage 2007;34(2):190-200.

Hauke D, Reiter-Theil S, Hoster E, Hiddemann W, Winkler EC. The role of relatives in decisions concerning life-prolonging treatment in patients with endstage malignant disorders: informants, advocates or surrogate decision-makers? Ann Oncol 2011;22(12):2667-74.

Houben $\mathrm{CH}$, Spruit MA, Groenen MT, Wouters EF, Janssen DJ. Efficacy of Advance Care Planning: A Systematic Review and Meta-Analysis. J Am Med Dir Assoc 2014;5(7):477-89. 
Hubert E, Schulte N, Belle S, Gerhardt A, Merx K, Hofmann WK et al. Cancer patients and advance directives: a survey of patients in a hematology and oncology outpatient clinic. Onkologie 2013;36(7-8):398-402.

In der Schmitten J. Advance care planning: putting an end to the agonizing perpetuation of a pointless debate. Onkologie 2013;36(7-8):395-6.

In der Schmitten J, Lex K, Mellert C, Rotharmel S, Wegscheider K, Marckmann G. Implementing an advance care planning program in German nursing homes: results of an inter-regionally controlled intervention trial. Dtsch Arztebl Int 2014;111(4):50-7.

In der Schmitten J, Rotharmel S, Mellert C, Rixen S, Hammes BJ, Briggs L et al. A complex regional intervention to implement advance care planning in one town's nursing homes: Protocol of a controlled inter-regional study. BMC Health Serv Res 2011;11:14.

Inoue M, Moorman SM. Does End-of-Life Planning Help Partners Become Better Surrogates? Gerontologist 2014. DOI 10.1093/geront/gnu031

Jackson JM, Rolnick SJ, Asche SE, Heinrich RL. Knowledge, attitudes, and preferences regarding advance directives among patients of a managed care organization. Am J Manag Care 2009;15(3):177-86.

Kirchhoff KT, Hammes BJ, Kehl KA, Briggs LA, Brown RL. Effect of a diseasespecific advance care planning intervention on end-of-life care. J Am Geriatr Soc 2012;60(5):946-50.

Knauss K. Patientenverfügungen aus Sicht unterschiedlicher Patientengruppen Strahlentherapie, Kardiologie, Unfallchirugie 2013. [Dissertation] Regensburg. Universität Regenburg

Knittel B. Betreuungsrecht. Kommentar und Rechtssammlung Bd.1. Neuwied: Luchterhand 2012.

Kuehlmeyer K, Borasio GD, Jox RJ. How family caregivers' medical and moral assumptions influence decision making for patients in the vegetative state: a qualitative interview study. J Med Ethics 2012;38(6):332-7.

Landeshauptstadt München. Konzept Pflegeüberleitung. http://www.muenchen.info/soz/pub/pdf/310_pflegeueberleitung_konzept.pdf (Zugriff am 3.12.2014)

Lang FR, Wagner GG. Patient living wills in Germany: conditions for their increase and reasons for refusal. Dtsch Med Wochenschr 2007;132(48):2558-62.

Langer S, Knorr J-U, Berg A. Umgang mit Patientenverfügungen: Probleme durch pauschale Formulierungen. Dtsch Arztebl International 2013;110(46):21868.

Lipp V. Handbuch der Vorsorgeverfügungen. München: Vahlen 2009.

Marckmann G, In der Schmitten J. Patientenverfügungen und Advance Care Planning: Internationale Erfahrungen. Zeitschrift für Medizinische Ethik 2013;59:213-27. 
May A, Niewohner S, Bickhardt J, Kreß H, Rothärmel S. Standards für die Beratung zu Patientenverfügungen. Zeitschrift für Ethik in der Medizin 2005;17:332-336.

Meeker MA, Jezewski MA. Family decision making at end of life. Palliat Support Care 2005;3(2):131-42.

Morrison RS, Olson E, Mertz KR, Meier DE. The inaccessibility of advance directives on transfer from ambulatory to acute care settings. Jama 1995;274(6):478-82.

Nauck F, Becker M, King C, Radbruch L, Voltz R, Jaspers B. To what extent are the wishes of a signatory reflected in their advance directive: a qualitative analysis. BMC Med Ethics 2014;15:52.

Noethen $\mathrm{M}$ et al. Hohe Kosten im Gesundheitswesen: Eine Frage des Alters?: Statistisches Bundesamt 2011. https://www.destatis.de/DE/Publikationen/WirtschaftStatistik/Gesundheitswesen/ FrageAlter.pdf;jsessionid=4C7EE9250AA14AE519F9B9F13FDF1B8E.cae1?_bl ob=publicationFile (Zugriff am 3.12.2014)

Palandt 0, Bassenge P, Brudermüller G. Bürgerliches Gesetzbuch. München: Beck 2014.

Sahm S, Will R, Hommel G. Attitudes towards and barriers to writing advance directives amongst cancer patients, healthy controls, and medical staff. J Med Ethics 2005a;31(8):437-40.

Sahm S, Will R, Hommel G. What are cancer patients' preferences about treatment at the end of life, and who should start talking about it? A comparison with healthy people and medical staff. Support Care Cancer 2005b;13(4):206-14.

Schickedanz AD, Schillinger D, Landefeld CS, Knight SJ, Williams BA, Sudore RL. A clinical framework for improving the advance care planning process: start with patients' self-identified barriers. J Am Geriatr Soc 2009;57(1):31-9.

Schildmann J, Dahmen B, Vollmann J. End-of-life practices of physicians in Germany. Dtsch Med Wochenschr 2014. DOI 10.1055/s-0034-1387410

Schildmann J, Winkler EC. Advance care planning in oncology - ethical and medical competences are needed. Oncol Res Treat 2014;37(1-2):60.

Schöffner M, Schmidt KW, Benzenhofer U, Sahm S. Living wills under close scrutiny: Medical consultation is indispensable. Dtsch Med Wochenschr 2012;137(10):487-90.

Schulte-Strathaus R. Palliativpass: Mehr Rechtssicherheit im Notfall. Dtsch Arztebl International 2014;111(21):916

Shalowitz DI, Garrett-Mayer E, Wendler D. The accuracy of surrogate decision makers: a systematic review. Arch Intern Med 2006;166(5):493-7.

Sharp T, Moran E, Kuhn I, Barclay S. Do the elderly have a voice? Advance care planning discussions with frail and older individuals: a systematic literature review and narrative synthesis. Br J Gen Pract 2013;63(615):e657-68. 
Silveira MJ, Kim SY, Langa KM. Advance directives and outcomes of surrogate decision making before death. N Engl J Med 2010;362(13):1211-8.

Silvester W, Fullam RS, Parslow RA, Lewis VJ, Sjanta R, Jackson L et al. Quality of advance care planning policy and practice in residential aged care facilities in Australia. BMJ Support Palliat Care 2013a;3(3):349-57.

Silvester W, Parslow RA, Lewis VJ, Fullam RS, Sjanta R, Jackson L et al. Development and evaluation of an aged care specific Advance Care Plan. BMJ Support Palliat Care 2013b;3(2):188-95

Simon A. Medizinethische Aspekte. In: Verrel T, Simon A (Hg.) Patientenverfügungen. Rechtliche und ethische Aspekte. Feiburg/München: Karl Alber 2010.

Simon A, May A. Leitlinien. http://www.ethikkomitee.de/leitlinien/index.html Zugriff 5.11.2014)

Sommer S, Marckmann G, Pentzek M, Wegscheider K, Abholz HH, in der Schmitten J. Advance directives in nursing homes: prevalence, validity, significance, and nursing staff adherence. Dtsch Arztebl Int 2012;109(37):577-83.

Sprung CL, Cohen SL, Sjokvist P, Baras M, Bulow HH, Hovilehto S et al. End-oflife practices in European intensive care units: the Ethicus Study. Jama 2003;290(6):790-7.

Stone L, Kinley J, Hockley J. Advance care planning in care homes: the experience of staff, residents, and family members. Int $\mathrm{J}$ Palliat Nurs 2013;19(11):550-7.

Tamayo-Velázquez M-I, Simón-Lorda P, Villegas-Portero R, Higueras-Callejón C, García-Gutiérrez J-F, Martínez-Pecino $F$ et al. Interventions to promote the use of advance directives: An overview of systematic reviews. Patient Education and Counseling 2010;80(1):10-20.

Tierney WM, Dexter PR, Gramelspacher GP, Perkins AJ, Zhou XH, Wolinsky FD. The effect of discussions about advance directives on patients' satisfaction with primary care. J Gen Intern Med 2001;16(1):32-40.

Tilden VP, Tolle SW, Nelson CA, Fields J. Family decision-making to withdraw life-sustaining treatments from hospitalized patients. Nurs Res 2001;50(2):10515.

Torjesen I. One in four GPs remains reluctant to initiate end of life discussions with patients. DOI 10.1136/bmj.g3195

Torke AM, Sachs GA, Helft PR, Montz K, Hui SL, Slaven JE et al. Scope and outcomes of surrogate decision making among hospitalized older adults. JAMA Intern Med 2014;174(3):370-7.

van Delden JJ, Lofmark R, Deliens L, Bosshard G, Norup M, Cecioni R et al. Donot-resuscitate decisions in six European countries. Crit Care Med 2006;34(6):1686-90.

van der Heide A, Deliens L, Faisst K, Nilstun T, Norup M, Paci E et al. End-of-life decision-making in six European countries: descriptive study. Lancet 2003;362(9381):345-50. 
van der Steen JT, van Soest-Poortvliet MC, Hallie-Heierman M, OnwuteakaPhilipsen BD, Deliens L, de Boer ME et al. Factors Associated with Initiation of Advance Care Planning in Dementia: A Systematic Review. J Alzheimers Dis 2014;40(3):743-57

van Oorschot B, Hausmann C, Köhler N, Leppert K, Schweitzer S, K. S. Patientenverfügungen aus Patientensicht. Ethik in der Medizin 2004;16:112-22.

van Oorschot B, Schuler M, Simon A, Flentje M. Advance directives: prevalence and attitudes of cancer patients receiving radiotherapy. Support Care Cancer 2012;20(11):2729-36.

van Wijmen MPS, Pasman HRW, Widdershoven GAM, Onwuteaka-Philipsen BD. Motivations, aims and communication around advance directives: A mixedmethods study into the perspective of their owners and the influence of a current illness. Patient Education and Counseling 2014;95(3):393-399.

Vig EK, Starks H, Taylor JS, Hopley EK, Fryer-Edwards K. Surviving surrogate decision-making: what helps and hampers the experience of making medical decisions for others. J Gen Intern Med 2007;22(9):1274-9.

Vig EK, Sudore RL, Berg KM, Fromme EK, Arnold RM. Responding to Surrogate Requests That Seem Inconsistent With a Patient's Living Will. Journal of Pain and Symptom Management 2011;42(5):777-782.

Vincent JL, Berré J, Creteur J. Withholding and withdrawing life prolonging treatment in the intensive care unit: a current European perspective. Chronic Respiratory Disease 2004;1(2):115-120.

Voltz R, Akabayashi A, Reese C, Ohi G, Saas H. End-of-life deicisions ans advance directives in palliative care: a cross-cultural survey of patients and health-care professionals. J Pain Symptom Manage 1998;16:152-62.

Wandrowski J, Schuster T, Strube W, Steger F. Medizinethische Kenntnisse und moralische Positionen von Ärztinnen und Ärzten aus Bayern. Dtsch Arztebl International 2012;109(8):141-7.

Weinick RM, Wilcox SR, Park ER, Griffey RT, Weissman JS. Use of advance directives for nursing home residents in the emergency department. Am J Hosp Palliat Care 2008;25(3):179-83.

Wendler D, Rid A. Systematic review: the effect on surrogates of making treatment decisions for others. Ann Intern Med 2011;154(5):336-46.

Wiese CH, Bartels U, Geyer A, Duttge G, Graf BM, Hanekop GG. The Gottingen palliative emergency card: improvement of emergency medical care for ambulatory palliative care patients. The "yellow card for rescue services". Dtsch Med Wochenschr 2008;133(18):972-6.

Wiese CH, Bartels UE, Ruppert DB, Graf BM, Hanekop GG. Prehospital emergency physicians' experiences with advance directives in Germany: a questionnaire-based multicenter study. Minerva Anestesiol 2011a;77(2):172-9.

Wiese CH, Duttge G, Taghavi M, Lassen CL, Meyer N, Andreesen R et al. Preventive crisis management in general outpatient palliative care - prospectively cross-sectional study of General Practitioners in Eastern Bavaria. Dtsch Med Wochenschr 2011b;136(45):2302-7. 
Wilke H-J, Wolf-Braun B, Zacharowski K. Gesundheitsvollmacht neues Projekt am Universitätsklinikum Frankfurt/M.: Vertrauensperson als kompetenter Partner für Ärzteteam. Dtsch Arztebl International 2014;111(8):300-2.

Wilkinson A. Advance Directives and Advance Care Planning: the US experience. In: Thomas K, Lobo B (Hg.) Advance Care Planning in End of Life Care. Oxford: Oxford University Press 2011, 189-204. 


\section{Anhang}

\subsection{Abbildungsverzeichnis}

Abbildung 1: Alle Berater ( $n=198)$ : Hauptveranlasser der Beratung.........21

Abbildung 2: Veranlasser der Beratung .............................................. 22

Abbildung 3: Alle Teilnehmer ( $n=198)$ : Gesundheitszustand der meisten

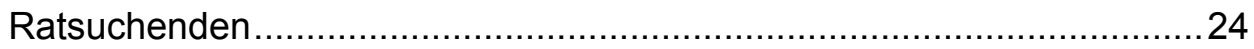

Abbildung 4: Gesundheitszustand der meisten Ratsuchenden ...............25

Abbildung 5: Alter der meisten Ratsuchenden...................................27

Abbildung 6: Alle Teilnehmer ( $n=198)$ : Anzahl der Gespräche die eine

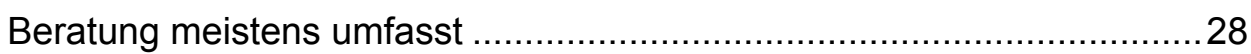

Abbildung 7: Anzahl der Gespräche innerhalb einer Beratung................29

Abbildung 8: Dauer der Beratung insgesamt ............................................30

Abbildung 9: Alle Teilnehmer ( $n=198)$ : Häufigkeit der Nennung der verschiedenen Formulare/Texte ..................................................... 31

Abbildung 10: Formulare/Texte anhand derer die Berater beraten .......... 32

Abbildung 11: Beratung anhand von durch den Ratsuchenden

mitgebrachten, i.d.R. vom Berater nicht verwendeten Formularen.

Abbildung 12: Häufigkeit der Fertigstellung der Patientenverfügung innerhalb der Beratung

Abbildung 13: Häufigkeit von Tipps zur Aufbewahrung der

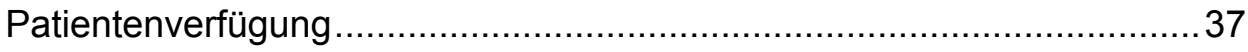

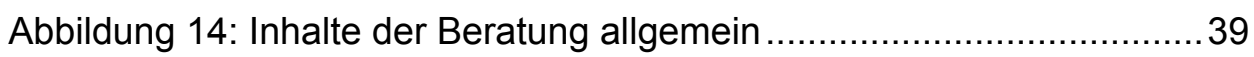

Abbildung 15: Häufigkeit von Information zu anderen

Vorsorgedokumenten

Abbildung 16: Notfallplanung

Abbildung 17: Hinweis auf die Teilnahmemöglichkeit zukünftiger Vertreter

und tatsächliche Teilnahme dieser Personen an der Beratung

Abbildung 18: Auswirkung der Einbeziehung des zukünftigen Vertreters auf den Berater 
Abbildung 19: Häufigkeit des Hinweises auf die Möglichkeit der Organspende

Abbildung 20: Themen, die im Hinblick auf eine Organspende angesprochen werden

Abbildung 21: Beteiligung bei der Ermittlung des Patientenwillens bei

Einwilligungsunfähigkeit.

Abbildung 22: Häufigkeit des Verweises an andere Berufsgruppen zur ergänzenden Beratung

Abbildung 23: Berufsgruppen, an die zur ergänzenden Beratung verwiesen wird

Abbildung 24: Wunsch nach verstärkter Zusammenarbeit/Austausch mit folgenden Berufsgruppen.

Abbildung 25: Bereiche, in denen mehr Austausch/Information gewünscht wird

Abbildung 26: Gewünschte Verbesserung des Austausches/der

Zusammenarbeit/der Information 60

Abbildung 27: Routinemäßiges Beratungsangebot ab einem bestimmten

Alter sinnvoll. 61

Abbildung 28: Erwerb der Kenntnisse für die Beratung 62

Abbildung 29: Schulung anhand bestimmter Formulare. 63

Abbildung 30: verwendete Schulungsformulare 64

Abbildung 31: Kostenpflicht der Beratung für den Ratsuchenden .65

Abbildung 32: Erhalt einer Vergütung 66

Abbildung 33: Einschätzung der Angemessenheit der Vergütung durch Berater, die eine Vergütung erhalten 66 


\subsection{Tabellenverzeichnis}

Tabelle 1: Pretest mit 34 Teilnehmern einer Schulung zur Beratung zur

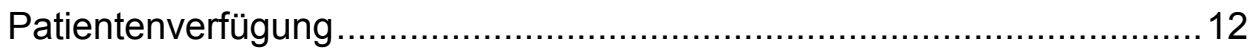

Tabelle 2: Funktion der teilnehmenden Berater..................................... 18

Tabelle 3: durchschnittliches Alter der Teilnehmer ....................................18

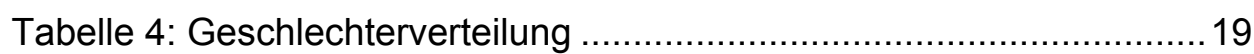

Tabelle 5: Jahre der Beratungstätigkeit ...............................................19

Tabelle 6: Anzahl der Einzelgespräche in 2012 ....................................20

Tabelle 7: Sonstige verwendete Formulare/Texte ................................ 34

Tabelle 8: Zusammenhang Anzahl Gespräche - Fertigstellung der

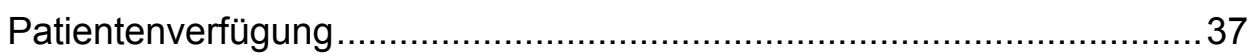

Tabelle 9: Tipps zur Aufbewahrung der Patientenverfügung im Einzelnen

Tabelle 10: Beratung von Menschen mit einer Lebenserwartung $<6$

Monate und Erstellung von Notfallplänen

Tabelle 11: Ärzte: Hinweis auf die Möglichkeit der Mitnahme des zukünftigen Vertreters - Auswirkung der Einbeziehung des zukünftigen Vertreters

Tabelle 12: Ärzte: Teilnahme des zukünftigen Vertreters an der Beratung Wirkung der Einbeziehung des zukünftigen Vertreters auf den Berater ...48

Tabelle 13: angemessene Höhe der Vergütung 67 


\subsection{Abkürzungsverzeichnis}

$$
\begin{array}{ll}
\text { ACP } & \text { = Advance Care Planning } \\
\text { BGB } & =\text { Bürgerliches Gesetzbuch } \\
\text { BT-Drucks. } & \text { Drucksache des Bundestages } \\
\mathrm{h} / \mathrm{i} & =\text { häufig/(fast) immer } \\
\mathrm{HV} & =\text { Hospizverein } \\
\mathrm{HV} \text { Mitarb. } & =\text { Mitarbeiter eines Hospizvereines } \\
\mathrm{k} . \mathrm{A} . & =\text { keine Angabe } \\
\mathrm{n} / \mathrm{s} & =\text { (fast) nie/selten } \\
\mathrm{PV} & =\text { Patientenverfügung } \\
\text { TN } & =\text { Teilnehmer } \\
\text { Überleitg. } & =\text { Überleitungsfachkraft }
\end{array}
$$




\section{Beratung zur Patientenverfügung}

Ein Blick in die Praxis

Eine Umfrage unter Hausärzten, Notaren, Überleitungsfachkräften sowie Mitarbeitern von

Betreuungs- und Hospizvereinen

im Raum München

durchgeführt vom

Institut für Ethik, Geschichte und Theorie der Medizin

Prof. Dr. Georg Marckmann, MPH

mit freundlicher Unterstützung durch

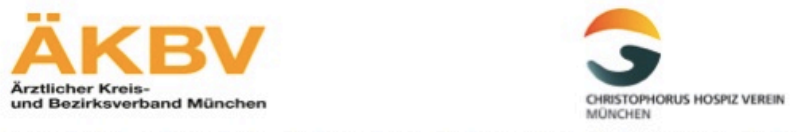

Prof. Dr. Georg Marckmann, MPH

Ludwig-Maximilians-Universität München

Institut für Ethik, Geschichte und Theorie

der Medizin

Email: Marckmann@lmu.de
Postanschrift:

Lessingstr. 2,

D-80336 München

http://www.egt.med.uni-muenchen.de
Kontakt:

Sabine.Petri@med.uni-muenchen.de

Fax: +49 (0)8921809972777

mob.: +49 (0) 1729099096

2 Hinweise, Datenschutz

Vielen Dank, dass Sie sich die Zeit für die Mitarbeit an dieser Umfrage nehmen!

Hier noch einige Hinweise zum Ausfüllen des Fragebogens

- Der Fragebogen bezieht sich auf Ihre aktuelle Beratungstätigkeit zu Patientenverfügungen.

- Es sollen Einzelberatungen erfasst werden, d.h. Gespräche mit dem Ratsuchenden (ggf. zusammen mit seinen Vertrauenspersonen), keine schriftlichen oder Gruppenberatungen.

- Bitte beantworten Sie, wenn möglich, auch alle Unterfragen

- Wo keine genauen Angaben möglich sind, sind Ihre Schätzungen erwünscht.

- Sofern Sie zur Zeit nicht beraten, bedanken wir uns herzlich für die Angabe der Gründe.

Bei Rückfragen wenden Sie sich bitte an Sabine.Petri@med.uni-muenchen.de

3 Datenschutz

Die Hinweise zum Schutz meiner Daten (bitte hier klicken) habe ich zur Kenntnis genommen und bin mit der Teilnahme an der Studie einverstanden

4 A1

Beraten Sie zur Patientenverfügung?

ja

nein

\subsection{A. Zur Beratungstätigkeit}

\section{A. Zur Beratungstätigkeit}
1. In welcher Funktion beraten Sie zur Patientenverfügung?
Mitarbeiter Betreuungsverein
hauptamtlicher Mitarbeiter Hospizverein
ehrenamtlicher Mitarbeiter Hospizverein
Überleitungsfachkraft
Notar
Hausarz 
2. Durch wen werden die Beratungen veranlasst?

$$
\text { (fast) nie }
$$

selten

häufig

(fast) immer

Sie selbst

den Ratsuchenden

Angehörige/Freunde

$\bigcirc$

0

eine versorgende Einrichtung

(Krankenhaus/Heim)

sonstige

$\bigcirc$

0

3. Wie ist der Gesundheitszustand der Ratsuchenden nach Ihrer Kenntnis?

$\begin{array}{lll}\text { (fast) nie } \quad \text { selten } & \text { häufig } & \text { (fast) immer }\end{array}$

\section{gesund}

schwer erkrankt

Lebenserwartung absehbar begrenzt $(<6$

Monate)

unbekannt

$\begin{array}{lll}0 & 0 & 0 \\ 0 & 0 & 0 \\ 0 & 0 & 0 \\ 0 & 0 & 0\end{array}$

\section{$5.1 .1 \quad$ A.2}

4. Zu welcher Altersgruppe gehören die meisten Ratsuchenden?

20 - 40 Jahre

$41-60$ Jahre

$61-80$ Jahre

> $>81$ Jahre

5. Wie viele Gespräche umfasst eine Beratung meistens?

1 Gespräch

2 Gespräche

3 Gespräche

4 und mehr Gespräche

6. Wie lange dauert eine Beratung meistens insgesamt ?

bis 30 Minuten

30-60 Minuten

60-90 Minuten

über 90 Minuten

5.1.2 A.3

7. Anhand welcher Formulare/Texte beraten Sie?

Mehrfachnennungen möglich

$\square$ Bundesministerium der Justiz

$\square$ Bayerisches Ministerium der Justiz

$\square$ Christliche Patientenverfügung

$\square$ selbst entwickelte Texte

$\square$ Es werden keine Formulare/Texte verwendet

8. Beraten Sie auch anhand von Ihnen i.d.R. nicht verwendeten Vordrucken, die durch den Ratsuchenden mitgebracht werden?

ja

nein

9. Wird die Patientenverfügung in der Regel mit dem Ratsuchenden innerhalb der Beratung fertig erstellt?

$\bigcirc$ ja

nein

10. Geben Sie in der Regel Hinweise für die Aufbewahrung der Patientenverfügung?

ja

nein

11. Wenn ja: Welche Tips geben Sie?

5.1.3 A.4

12. Wie häufig beinhalten Ihre Gespräche eine Beratung zu folgenden Themen?

$\begin{array}{lll}\text { (fast) nie } \quad \text { selten } & \text { häufig } & \text { (fast) immer }\end{array}$

Flüssigkeit und Ernährung am Lebensende unheilbare, tödlich verlaufende Krankheit irreversible Bewusstlosigkeit

psychiatrische Erkrankungen

akuter medizinischer Notfall

13. Wie häufig informieren Sie über

$\begin{array}{llll}0 & 0 & 0 & 0 \\ 0 & 0 & 0 & 0 \\ 0 & 0 & 0 & 0 \\ 0 & 0 & 0 & 0 \\ 0 & 0 & 0 & 0\end{array}$




\begin{tabular}{|c|c|c|c|c|}
\hline & (ldst) me & selterl & Ilauny & (last) mипиег \\
\hline Vorsorgevollmacht & 0 & 0 & 0 & 0 \\
\hline Betreuungsverfügung & 0 & 0 & 0 & O \\
\hline Möglichkeit eines Notfallplanes & 0 & 0 & 0 & 0 \\
\hline \multicolumn{5}{|c|}{ 14. Wie häufig weisen Sie auf die Möglichkeit hin, einen zukünftigen Vorsorgebevollmächtigten/Betreuer zur Beratung mitzubringen? } \\
\hline (fast) nie & & häufig & (fast) immer & \\
\hline $\mathrm{O}$ & & 0 & $\bigcirc$ & \\
\hline \multicolumn{5}{|c|}{ 15. Wie häufig nimmt ein zukünftiger Vorsorgebevollmächtigter/Betreuer an der Beratung teil? } \\
\hline (fast) nie & & häufig & (fast) immer & \\
\hline$\bigcirc$ & & 0 & O & \\
\hline \multicolumn{5}{|c|}{ 16. Empfinden Sie die Einbeziehung einer solchen Person für Sie persönlich als eher } \\
\hline hilfreich & störend & oh & & \\
\hline$\bigcirc$ & O & & & \\
\hline \multicolumn{5}{|l|}{$5.1 .4 \quad$ A.5 } \\
\hline \multicolumn{5}{|c|}{ 17. Wie häufig weisen Sie auf die Möglichkeit einer Organspende hin? } \\
\hline (fast) nie & & häufig & (fast) immer & \\
\hline 0 & & 0 & 0 & \\
\hline \multicolumn{5}{|c|}{ 18. Wenn ja: Wie häufig sprechen Sie dabei folgende Punkte an? } \\
\hline & (fast) nie & selten & häufig & (fast) immer \\
\hline $\begin{array}{l}\text { Hirntod als Voraussetzung für die } \\
\text { Organspende }\end{array}$ & 0 & 0 & 0 & 0 \\
\hline $\begin{array}{l}\text { Intensivstation als Voraussetzung für die } \\
\text { Hirntodfeststellung }\end{array}$ & 0 & O & 0 & O \\
\hline $\begin{array}{l}\text { Mögliche Konflikte zwischen } \\
\text { Patientenverfügung und Organspende }\end{array}$ & 0 & $\bigcirc$ & 0 & O \\
\hline $\begin{array}{l}\text { Möglichkeit der Prioritätensetzung für solche } \\
\text { Konflikte }\end{array}$ & $\bigcirc$ & O & O & O \\
\hline \multicolumn{5}{|c|}{ 19. Erarbeiten Sie auch Notfallpläne mit den Ratsuchenden? } \\
\hline (fast) nie & & häufig & (fast) immer & \\
\hline 0 & & 0 & 0 & \\
\hline
\end{tabular}

\subsection{Zum Umfeld der Beratung}

\section{B. Zum Umfeld der Beratung}

1. Werden Sie auch bei medizinischen Entscheidungen bei Einwilligungsunfähigkeit des Patienten zur Ermittlung des Patientenwillens zugezogen?

ja

nein

2. Verweisen Sie zur ergänzenden Beratung an andere an der Vorsorgeplanung beteiligte Berufsgruppen?
(fast) nie
selten
häufig
(fast) immer

3. Wenn ja: An wen verweisen Sie?

Mehrfachnennungen möglich

$\square$ Arzt

$\square$ Notar

$\square$ Pflege

$\square$ Betreuungsverein/-stelle

$\square$ Hospizverein

0

5.2.1 B. 2

4. Mit welcher anderen an der Vorsorgeplanung beteiligten Berufsgruppe wünschen Sie sich verstärkte Zusammenarbeit/Austausch? Mehrfachnennungen möglich

$\square$ Arzt

$\square$ Notar

$\square$ Pflege

$\square$ Betreuer

$\square$ Hospizverein

$\square$ mit keiner anderen Berufsgruppe

5. In welchem Bereich wünschen Sie sich mehr Austausch/Information?

Mehrfachnennungen möglich

$\square$ Fragen der Kommunikation mit den Ratsuchenden

$\square$ medizinische Fragen 


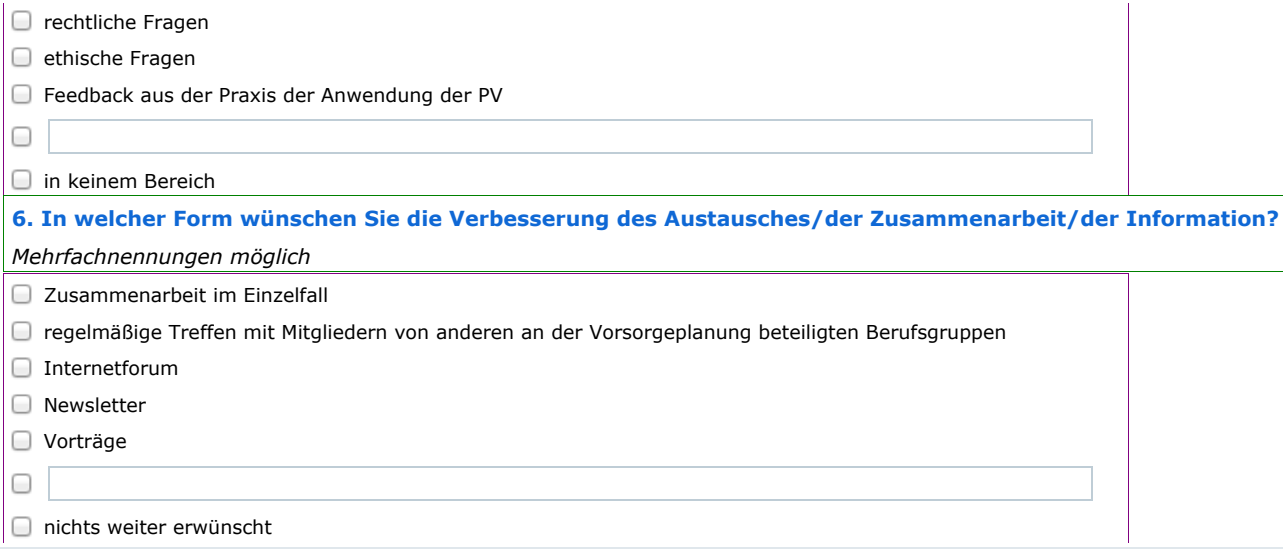

5.2.2 B3

7. Halten Sie ein routinemäßiges Angebot einer Beratung zur Patientenverfügung ab einem bestimmten Alter für sinnvoll?

$\bigcirc$ ja

nein

8. Was ist Ihr persönliches, besonderes Anliegen im Hinblick auf die Beratung zur Patientenverfügung?

\subsection{Zur Person des Beraters}

\section{Zur Person des Beraters}

1. Seit wie vielen Jahren üben Sie Ihre Beratungstätigkeit aus?

2. Wie viele Einzelgespräche haben Sie in $\mathbf{2 0 1 2}$ geführt? auch Schätzungen erwünscht

\subsubsection{2}

3. Wie haben Sie Ihre Kenntnisse für die Beratungstätigkeit erworben?

Selbststudium

$\square$ Studium

$\square$ Schulung/Weiterbildung bei

4. Wurden Sie anhand bestimmter Formulare geschult?

ja

nein

5. Wenn ja: Anhand welcher Formulare wurden Sie geschult? Mehrfachnennungen möglich

$\square$ Bundesministerium der Justiz

$\square$ Bay. Staatsministerium der Justiz

$\square$ Christliche Patientenverfügung

$\square$ sonstige

$5.3 .2 \quad$ C.3

6. Erfolgt die Beratung für den Ratsuchenden
0 kostenpflichtig
unentgeltlich

7. Erhalten Sie eine Vergütung für die Beratung?

ja

nein

8. Wenn ja: Halten Sie die Vergütung für angemessen?

O

9. Wenn nein: Welche Höhe der Vergütung wäre angemessen?

5.3.3 C. 4

10. In welchem Jahr sind Sie geboren? 
11. Geschlecht

12. Bitte teilen Sie uns die Postleitzahl Ihrer Praxisanschrift mit

Dieses ist die letzte Frage. Mit dem Öffnen der nächsten Seite wird der Fragebogen versendet. Er kann dann nicht wieder geöffnet werden.

6.1 Nicht-Berater Auskunft

1. In welcher Funktion sind Sie tätig?

$\square$ Hausarzt

$\square$ Notar

$\square$ Überleitungsfachkraft

Mitarbeiter Betreuungsverein

$\square$ ehrenamtlicher Mitarbeiter Hospizverein

$\square$ hauptberuflicher Mitarbeiter Hospizverein

2. Aus welchem Grund beraten Sie nicht zur Patientenverfügung? Mehrfachnennungen möglich

$\square$ Beratung zur PV fällt nicht meinen Aufgabenbereich

$\square$ Beratung wird nicht nachgefragt

$\square$ Beratung zur PV wird nicht vergütet

$\square$ Das Thema liegt mir nicht

$\square$ Ich fühle mich nicht ausreichend kompetent

3. Wer berät in Ihrer Institution/Praxis zur Patientenverfügung?

○

Es wird nicht zur Patientenverfügung beraten

4. Würden Sie gerne zur Patientenverfügung beraten?

$\bigcirc$ ja

nein

bin unentschlossen

5. Was bräuchten Sie, um zu beraten?

$\square$ z.B.:

$\square$ kein Bedarf

6.2 AuskunftNB 2

7. Geschlecht

weiblich männlich

O

6. In welchem Jahr sind Sie geboren?

9. Bitte teilen Sie uns die Postleitzahl Ihrer Praxisanschrift mit.

8. Was ist Ihr persönliches, besonderes Anliegen im Hinblick auf die Beratung zur Patientenverfügung?

Dieses ist die letzte Frage. Mit dem Öffnen der nächsten Seite wird der Fragebogen versendet. Er kann dann nicht wieder geöffnet werden.

7 Endseite

\section{Herzlichen Dank für Ihre Teilnahme!}




\section{Danksagung}

Diese Arbeit wäre ohne die Unterstützung vieler Menschen nicht möglich geworden.

Besonderer Dank gilt Prof. Dr. med. Georg Marckmann, der mir die Möglichkeit gab, die Studie im Rahmen einer Dissertation durchzuführen und sie stets mit wertvollen Anregungen und Diskussionen begleitete.

Eine große Hilfestellung war der Austausch mit meinen Kolleginnen und Kollegen am Institut für Ethik, Geschichte und Theorie der Medizin, denen ich hiermit herzlich danken möchte. Hervorzuheben ist dabei das Engagement von Dr. Katja Kühlmeyer, die mir insbesondere in methodischen Fragen zur Seite stand. Viele Anregungen zum Themenkomplex Organspende gab PD Dr. Dr. Ralf Jox. Sandra Thiersch ließ mich an ihren Kenntnissen in der SPSS-Auswertung teilhaben.

Für die hilfreichen Hinweise bei der statistischen Auswertung der Daten danke ich Herrn Dr. Crispin vom Institut für Medizinische Informationsverarbeitung, Biometrie und Epidemiologie der Ludwig-Maximilians- Universität.

Danken möchte ich vor allem den Teilnehmern der Studie und denen, die den Kontakt zu den Teilnehmern ermöglicht haben. Dieser Dank richtet sich insbesondere an Herrn Sepp Raischl, Herrn Dr. Gregor Scheible, Herrn Notar Dr. Hans-Frieder Krauß, Herrn Notar Dr. Thiede, die vier Münchner Hospizvereine, den Ärztlichen Kreis- und Bezirksverband sowie den Bayerischen Hausärzteverband.

Herrn Karlo Heßdörfer möchte ich an dieser Stelle herzlich für die immer anregenden Diskussionen und das Korrekturlesen der Arbeit danken.

Wesentliche Grundlage für die Fertigstellung dieser Studie war die beständige Ermutigung und der stabile Rückhalt durch meine Familie und Freunde, denen ich dafür sehr dankbar bin.

Von ganzem Herzen danke ich meinem Mann für seine große Geduld, sein unerschütterliches Vertrauen und die konstruktive Kritik bei der Verfassung der Arbeit.

Auch den vielen anderen Menschen, die mich begleiteten und zum Gelingen meiner Arbeit beitrugen, aber nicht namentlich genannt wurden, sei herzlich gedankt! 


\section{Eidesstattliche Versicherung}

Ich, Sabine Petri, erkläre hiermit an Eides statt,

dass ich die vorliegende Dissertation mit dem Thema

Beratung zur Patientenverfügung

Eine empirisch-ethische Untersuchung der Beratung zur Patientenverfügung durch ausgewählte Berater in der Region München

selbstständig verfasst, mich außer den angegeben keiner weiteren Hilfsmittel bedient und alle Erkenntnisse, die aus dem Schrifttum ganz oder annähernd übernommen sind, als solche kenntlich gemacht und nach ihrer Herkunft unter Bezeichnung der Fundstelle einzeln nachgewiesen habe.

Ich erkläre des Weiteren, dass die hier vorgelegte Dissertation nicht in gleicher oder in ähnlicher Form bei einer anderen Stelle zur Erlangung eines akademischen Grades eingereicht wurde.

München, den 28.01.2015 